Equações diferenciais ordinárias generalizadas lineares e aplicações às equações diferenciais funcionais lineares

\author{
Rodolfo Collegari
}





\title{
Equações diferenciais ordinárias generalizadas lineares e aplicações às equações diferenciais funcionais lineares
}

\author{
Rodolfo Collegari
}

Orientadora: Profa. Dra. Márcia Cristina Anderson Braz Federson

Tese apresentada ao Instituto de Ciências Matemáticas e de Computação - ICMC-USP, como parte dos requisitos para obtenção do título de Doutor em Ciências - Matemática . VERSÃO REVISADA 
Ficha catalográfica elaborada pela Biblioteca Prof. Achille Bassi e Seção Técnica de Informática, ICMC/USP, com os dados fornecidos pelo(a) autor(a)

\section{Collegari, Rodolfo}

C697e Equações diferenciais ordinárias generalizadas lineares e aplicações às equações diferenciais funcionais lineares / Rodolfo Collegari; orientadora Márcia Cristina Anderson Braz Federson. -- São Carlos, 2014. 94 p.

Tese (Doutorado - Programa de Pós-Graduação em Matemática) -- Instituto de Ciências Matemáticas e de Computação, Universidade de São Paulo, 2014.

1. equações diferenciais ordinárias generalizadas. 2. equações diferenciais funcionais. 3. fórmula da variação das constantes. 4. integral de Kurzweil. I. Federson, Márcia Cristina Anderson Braz, orient. II. Título. 
"Eu acredito demais na sorte.

E tenho constatado que, quanto mais duro eu trabalho, mais sorte eu tenho". (Thomas Jefferson)

Aos meus pais. 



\section{Agradecimentos}

Primeiramente, quero agradecer a Deus por me dar saúde para viver e por me dar forças para percorrer esta jornada.

Agradeço aos meus pais, por todo o esforço e sacrifício que fizeram para me manter em São Carlos nos meus primeiros anos longe de casa, pelo amor e carinho que sempre me deram e por sempre acreditarem em mim. Muito obrigado, amo vocês.

Agradeço ao meu irmão, que se juntou a mim em São Carlos para seus estudos. Foi muito bom tê-lo por perto todo esse tempo, passamos muitos momentos legais aqui. A nossa convivência sempre muito boa, obrigado pelo seu companheirismo.

Agradeço a minha família, que sempre esteve junto comigo, me apoiando e dando todo o suporte para eu realizar meus estudos. Muito obrigado por sempre me receberem com muito amor e carinho.

Agradeço a minha namorada Luciana, uma pessoa maravilhosa que entrou na minha vida e só me trouxe felicidades. Muito obrigado por todo o seu amor, carinho, compreensão, paciência, confiança e incentivo, você contribuiu muito nesse trabalho, sempre me passando todo seu otimismo e confiança, principalmente quando as coisas não davam certo. Seu incentivo sempre me fazia voltar ainda mais disposto para resolver os problemas. Te amo.

Agradeço a todos os meus amigos que fiz ao longo desses anos, pelos momentos que passamos juntos, seja estudando ou nos divertindo. Em especial, agradeço aos meus amigos Matheus e Henrique (Mineiro), por me ouvirem em diversos momentos e por me ajudarem em problemas ao longo deste trabalho.

Agradeço a todos os meus professores do ICMC, que contribuíram para minha formação acadêmica e, também, agradeço a todos os funcionários do ICMC, que são os principais responsáveis por fazer desse instituto um excelente lugar para se estudar.

Agradeço a minha orientadora Márcia Federson, por ter me orientado todos esses anos, desde a iniciação científica na graduação. A sua contribuição foi muito importante para o 
meu crescimento acadêmico, muito obrigado por sempre acreditar em mim.

Por fim, agradeço a Fapesp pelo suporte financeiro no decorrer deste trabalho. 


\section{Abstract}

In this work, we present a variation-of-constants formula for linear generalized ordinary differential equations in Banach spaces. More specifically, we are interested in establishing a relation between the solutions of the Cauchy problem for a linear generalized ordinary differential equation

$$
\frac{d x}{d \tau}=D[A(t) x], \quad x\left(t_{0}\right)=\tilde{x}
$$

and the solutions of the perturbed Cauchy problem

$$
\frac{d x}{d \tau}=D[A(t) x+F(x, t)], \quad x\left(t_{0}\right)=\widetilde{x},
$$

where the functions involved are generalized Perron integrable and, hence, admit many discontinuities and oscillations. We also prove that there exists a one-to-one correspondence between the Cauchy problem for a linear functional differential equations of the form

$$
\left\{\begin{array}{l}
\dot{y}=\mathscr{L}(t) y_{t} \\
y_{t_{0}}=\varphi
\end{array}\right.
$$

where $\mathscr{L}$ is a bounded linear operator and $\varphi$ is a regulated function, and a certain class of linear generalized ordinary differential equations. As a consequence, we are able to obtain a variation-of-constants formula relating the solutions of the linear functional differential equation and the solutions of the perturbed problem

$$
\left\{\begin{array}{l}
\dot{y}=\mathscr{L}(t) y_{t}+f\left(y_{t}, t\right), \\
y_{t_{0}}=\varphi,
\end{array}\right.
$$

where the application $t \mapsto f\left(y_{t}, t\right)$ is Perron integrable, with $t$ in an interval of $\mathbb{R}$, for each regulated function $y$. 



\section{Resumo}

Neste trabalho, apresentamos uma fórmula da variação das constantes para EDOs generalizadas lineares em espaços de Banach. Mais especificamente, estamos interessados em estabelecer uma relação entre as soluções do problema de Cauchy para uma EDO generalizada linear

$$
\frac{d x}{d \tau}=D[A(t) x], \quad x\left(t_{0}\right)=\widetilde{x}
$$

e as soluções do problema de Cauchy perturbado

$$
\frac{d x}{d \tau}=D[A(t) x+F(x, t)], \quad x\left(t_{0}\right)=\widetilde{x}
$$

em que as funções envolvidas são Perron integráveis e, portanto, admitem muitas descontinuidades e oscilações. Também provamos a existência de uma correspondência biunívoca entre o problema de Cauchy para uma EDF linear da forma

$$
\left\{\begin{array}{l}
\dot{y}=\mathscr{L}(t) y_{t}, \\
y_{t_{0}}=\varphi
\end{array}\right.
$$

em que $\mathscr{L}$ é um operador linear e limitado e $\varphi$ é uma função regrada, e uma certa classe de EDOs generalizadas lineares. Como consequência, obtemos uma fórmula da variação das constantes relacionando as soluções da EDF linear e as soluções do problema perturbado

$$
\left\{\begin{array}{l}
\dot{y}=\mathscr{L}(t) y_{t}+f\left(y_{t}, t\right), \\
y_{t_{0}}=\varphi,
\end{array}\right.
$$

em que a aplicação $t \mapsto f\left(y_{t}, t\right)$ é Perron integrável, com $t$ em um intervalo de $\mathbb{R}$, para cada função regrada $y$. 



\section{Sumário}

1 A integral de Kurzweil $\quad 19$

1.1 Funções regradas e funções de variação limitada . . . . . . . . . . . . . . . . . 19

1.2 A integral de Kurzweil em espaço de Banach . . . . . . . . . . . . . . . . . 23

1.3 A integral de Kurzweil-Cauchy em espaço de Banach . . . . . . . . . . . . . . . 29

1.4 Teorema de Representação de Riesz . . . . . . . . . . . . . . . . . . . . . 37

2 EDOs generalizadas $\quad 43$

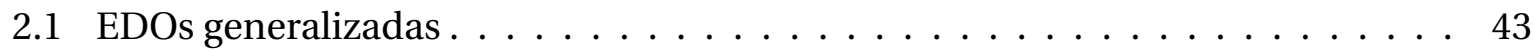

2.2 EDOs generalizadas lineares $\ldots \ldots \ldots \ldots \ldots \ldots \ldots \ldots \ldots$

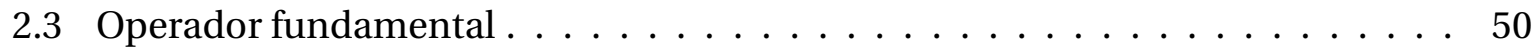

2.4 Fórmula da variação das constantes . . . . . . . . . . . . . . . . 57

3 EDFs no contexto de EDOs generalizadas $\quad 65$

4 Aplicações $\quad 77$

4.1 Fórmula da variação das constantes para EDFs . . . . . . . . . . . . 77

4.2 Fórmula da variação das constantes para EDFs com impulso . . . . . . . . . . 86

4.3 Fórmula da variação das constantes para EDFNs em medida . . . . . . . . . . 88 



\section{Introdução}

Sabemos que a classe de funções integráveis segundo Riemann é muito restrita e não possui boas propriedades de convergência. No final do século XIX, foram feitas várias tentativas no sentido de remediar alguns defeitos dessa integral e, dentre elas, merece destaque a trabalho de Henry Lebesgue de 1902. Sua teoria ampliou muito a classe das funções integráveis e, além disso, deu condições mais satisfatórias de convergência. No entanto, a integral de Lebesgue não contém suas integrais impróprias. Outro inconveniente dessa teoria diz respeito às condições necessárias para que a derivada de uma função seja integrável.

Em resposta a todos esses problemas, procurou-se criar um conceito de integral que generalizasse a integral de Lebesgue. Essa meta foi alcançada por Arnold Denjoy em 1912 e, dois anos mais tarde, por Oscar Perron (veja [7] e [32]). Apesar da definição das integrais de Denjoy e Perron serem bastante diferentes, provou-se que ambas são equivalentes.

Nas décadas de 50 e 60 do século passado, o matemático tcheco Jaroslav Kurzweil e o matemático inglês Ralph Henstock conseguiram, de modo independente, apresentar uma definição para as integrais de Denjoy e de Perron baseada em somas de Riemann. Além de ser equivalente aos conceitos de integral segundo Denjoy e Perron, a definição de integral de Kurzweil-Henstock é notavelmente mais simples. Veja [18]. Hoje, são conhecidas outras possibilidades de extensão da integral de Lebesgue que estão contidas propriamente no espaço de funções Kurzweil-Henstock integráveis. Veja [5] e [28].

A fim de generalizar certos resultados sobre dependência contínua de soluções de EDOs 
com respeito aos dados iniciais, J. Kurzweil introduziu, em 1957, a noção de equações diferenciais ordinárias generalizadas para funções a valores em espaços euclideanos e de Banach. Esta generalização da noção de EDO inclui a noção de integral de Perron generalizada ou integral de Kurzweil como é chamada hoje em dia. Nós nos referimos a estas novas equações como EDOs generalizadas. Veja as referências [3], [23] a [27] e, também, [33].

A correspondência entre EDOs generalizadas e EDOs clássicas é bem simples. Sabe-se que o sistema ordinário

$$
\dot{x}=f(x, t)
$$

onde $\dot{x}=d x / d t, \Omega \subset \mathbb{R}^{n}$ é aberto e $f: \Omega \times \mathbb{R} \rightarrow \mathbb{R}$, é equivalente à equação integral

$$
x(t)=x\left(t_{0}\right)+\int_{t_{0}}^{t} f(x(\tau), \tau) d \tau, \quad t \geq t_{0}
$$

quando a integral existe em algum sentido. Sabe-se, também, que se a integral em (0.2) for no sentido de Riemann, Lebesgue (com a definição equivalente de McShane) ou KurzweilHenstock, por exemplo, então ela pode ser aproximada por uma soma da forma

$$
\sum_{i=1}^{m} f\left(x\left(\tau_{i}\right), \tau_{i}\right)\left[s_{i}-s_{i-1}\right]
$$

onde $t_{0}=s_{0} \leq s_{1} \leq \ldots \leq s_{m}=t$ é uma partição fina do intervalo $\left[t_{0}, t\right]$ e, para cada $i=$ $1,2, \ldots, m, \tau_{i}$ está suficientemente "perto" do intervalo $\left[s_{i-1}, s_{i}\right]$. Alternativamente, se definirmos

$$
F(x, t)=\int_{t_{0}}^{t} f(x, s) d s, \quad(x, t) \in \Omega \times \mathbb{R}
$$

então a integral em (0.2) poderá ser aproximada por

$$
\sum_{i=1}^{m} \int_{s_{i-1}}^{s_{i}} f\left(x\left(\tau_{i}\right), \sigma\right) d \sigma=\sum_{i=1}^{m}\left[F\left(x\left(\tau_{i}\right), s_{i}\right)-F\left(x\left(\tau_{i}\right), s_{i-1}\right)\right] .
$$

Neste caso, o lado direito de (0.3) aproxima-se da integral não-absoluta de Kurzweil a qual, 
quando considerada em (0.2), dá origem a uma equação diferencial do tipo $(0.1)$, porém num sentido mais amplo. Tal equação diferencial é conhecida como equação diferencial ordinária generalizada. Escreveremos EDOs generalizadas por simplicidade. Veja [33]. Assim como para EDOs clássicas (veja [3]), uma das construções básicas na aplicação da teoria de dinâmica topológica a EDOs não-autônomas do tipo

$$
\dot{x}=f(x, t)
$$

é dada considerando-se os pontos limites (quando $|s| \rightarrow \infty$ ) das transladadas $f_{s}$, onde

$$
f_{s}(x, t)=f(x, t+s) \text {. }
$$

Entretanto, um fenômeno interessante pode ocorrer, se $f$ ou sua integral indefinida satisfizerem condições do tipo Carathéodory e Lipschitz mais gerais do que as usuais: as equações limites podem não ser EDOs. Um exemplo para este fato é apresentado em [3]. Nestas condições, identificando-se esta classe de EDOs com uma classe de EDOs generalizadas apropriada, é possível construir um fluxo local para um problema de valor inicial para esta classe de EDOs generalizadas e diversas aplicações podem ser obtidas e traduzidas para o contexto de EDOs clássicas. Veja [1] e [16].

Em [16], foi provado que EDFRs podem ser identificadas com EDOs generalizadas e algumas aplicações provenientes desta relação foram investigadas. Em [14], com a colaboração do professor Štefan Schwabik, foi provado que EDFRs sujeitas a efeitos impulsivos também podem ser relacionadas com certa classe de EDOs generalizadas numa correspondência biunívoca. A partir destes trabalhos, ficou evidente a importância de desenvolvermos a teoria de EDOs generalizadas para utilizar os resultados obtidos em investigações nas teorias de EDOs, EDFRs, Equações Diferenciais em Medida e, mais recentemente, Equações Dinâmicas em Escalas Temporais (veja as referências [4], [9], [10] e [11]) e Equações Diferenciais do tipo neutro (veja [8]). 
A teoria das Equações Diferenciais Funcionais Retardadas (EDFRs) é um ramo das Equações Diferenciais Funcionais (EDFs). Uma das razões do nosso interesse em EDFRs é por elas se constituírem em exemplos de sistemas dinâmicos de dimensão infinita, apresentando dinâmica complexa. Do ponto de vista das aplicações, o interesse em EDFRs está em que, para muitos fenômenos naturais, notadamente físicos e biológicos, a aplicação do princípio de causalidade envolve um lapso de tempo entre causa e efeito. Desta forma, os modelos determinísticos mais realistas são frequentemente descritos por equações que envolvem retardos. Como mencionamos acima, uma das ferramentas principais na investigação e resolução de problemas da teoria de EDFs será a utilização das EDOs generalizadas.

A correspondência entre EDOs generalizadas e EDFRs impulsivas foi estabelecida no artigo [14] para o caso de impulsos pré-fixados e no artigo [2] para o caso de impulsos em tempo variável.

Neste trabalho, consideramos o seguinte problema de valor inicial para uma equação diferencial funcional linear

$$
\left\{\begin{array}{l}
\dot{y}=\mathscr{L}(t) y_{t} \\
y_{t_{0}}=\phi
\end{array}\right.
$$

e também o correspondente problema perturbado

$$
\left\{\begin{array}{l}
\dot{y}=\mathscr{L}(t) y_{t}+f\left(y_{t}, t\right) \\
y_{t_{0}}=\phi
\end{array}\right.
$$

em que $\phi \in G\left([-r, 0], \mathbb{R}^{n}\right)$ e $\mathscr{L}(t): G\left([-r, 0], \mathbb{R}^{n}\right) \rightarrow \mathbb{R}^{n}$ é linear e limitada para todo $t \in\left[t_{0}, t_{0}+\right.$ $\sigma]$, isto é, $\mathscr{L}(t) \in L\left(G\left([-r, 0], \mathbb{R}^{n}\right), \mathbb{R}^{n}\right)$ para todo $t \in\left[t_{0}, t_{0}+\sigma\right]$ e $f: G\left([-r, 0], \mathbb{R}^{n}\right) \times\left[t_{0}, t_{0}+\sigma\right] \rightarrow$ $\mathbb{R}^{n}$ e as funções envolvidas são integráveis no sentido de Kurzweil.

Nosso objetivo nesse trabalho é encontrar uma fórmula da variação das constantes para a EDF perturbada (0.4). Isto será feito usando a teoria das EDOs generalizadas para a qual, também provamos uma fórmula da variação das constantes mais geral que as encontradas na literatura. 
Este trabalho está dividido em 4 capítulos. Iniciaremos vendo a definição e propriedades básicas da integral de Kurzweil. Como mencionamos acima, essa integral é muito importante para o desenvolvimento desse trabalho, pois é através dela que iremos definir as equações diferenciais ordinárias generalizadas no Capítulo 2. Ainda no primeiro capítulo, introduzimos uma nova integral que chamaremos de integral de Kurzweil-Cauchy. Veremos que propriedades equivalentes às propriedades básicas da integral de Kurzweil valem para essa nova integral. No final do capítulo, usando a integral de Kurzweil-Cauchy, apresentaremos uma nova versão para o Teorema de Representação de Riesz para funções regradas.

No Capítulo 2, apresentaremos as equações diferencias ordinárias generalizadas e um pouco da teoria básica dessa classe de equações. Além da teoria básica geral de EDOs generalizadas, estudaremos o caso particular das EDOs generalizadas lineares, apresentaremos o operador fundamental associado a uma EDO generalizada linear e veremos algumas de suas propriedades. Na sequência, apresentaremos alguns resultados auxiliares, tais como uma versão da fórmula de Dirichilet para a integral de Kurzweil, encerrando o capítulo com uma fórmula da variação das constantes pra uma EDO generalizada linear perturbada. Esses últimos resultados são novos e podem ser encontrados em [6].

O terceiro capítulo relaciona as EDFs lineares com EDOs generalizadas lineares. Essa correspondência é fundamental para o obtenção da fórmula da variação das constantes para EDFs lineares perturbadas. Iniciaremos vendo a construção dos operadores envolvidos na EDO generalizada linear associada à EDF linear e, no fim do capítulo, apresentamos tal correspondência.

O último capítulo trata da fórmula da variação das constantes para equações com retardamento. Nele, aplicaremos a fórmula da variação das constantes para EDOs generalizadas obtida no Capítulo 2 em outros tipos de equação, tais como EDFs, EDFs com impulso e EDFNs em medida. Esses resultados reforçam a importância das EDOs generalizadas, que têm sido uma grande ferramenta para generalização de diversos resultados em equações diferencias, tais como existência e unicidade de soluções, dependência contínua das soluções 
com relação aos dados iniciais, estabilidade de soluções, entre outros. 


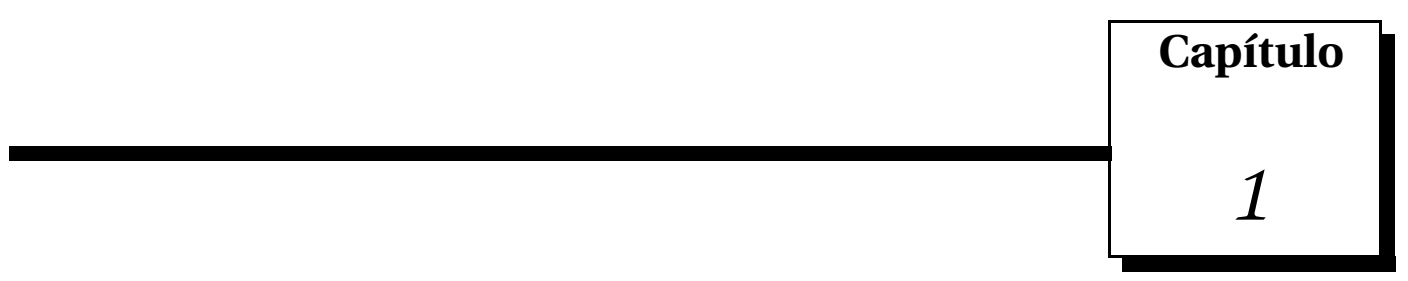

\section{A integral de Kurzweil}

O primeiro capítulo deste trabalho está dividido em quatro partes. Começaremos definindo as classes das funções regradas e também das funções de variação limitadas de intervalo compacto $[a, b] \subset \mathbb{R}$ em um espaço de Banach $X$. Essas duas classes de funções são muito importantes no contexto da integral de Kurzweil que será definida na sequência, juntamente com algumas propriedades básicas de integração. Na seção seguinte, vamos introduzir uma nova integral que chamaremos de integral de Kurzweil-Cauchy. Também para esta integral, veremos algumas propriedades básicas. Por fim, através da integral de Kurzweil-Cauchy, apresentaremos uma versão do Teorema de Representação de Riesz para funções regradas.

\subsection{Funções regradas e funções de variação limitada}

Nesta primeira seção, vamos definir duas classes de funções que serão muito importantes ao longo do trabalho, principalmente por estarem relacionas com a integral de Kurzweil que veremos na sequência.

Sejam $X$ um espaço de Banach com norma $\|\cdot\|$ e $a, b$ números reais tais que $-\infty<a<b<\infty$. 
Uma função $f:[a, b] \rightarrow X$ será dita regrada, se os limites laterais

$$
\lim _{s \rightarrow t^{-}} f(s), \quad t \in(a, b], \quad e \quad \lim _{s \rightarrow t^{+}} f(s), \quad t \in[a, b)
$$

existirem. Neste caso, denotamos $f\left(t^{-}\right)=\lim _{s \rightarrow t^{-}} f(s)$ e $f\left(t^{+}\right)=\lim _{s \rightarrow t^{+}} f(s)$.

O espaço das funções regradas $f:[a, b] \rightarrow X$ será um espaço de Banach, se considerarmos a norma do supremo $\|f\|_{\infty}=\sup _{t \in[a, b]}\|f(t)\|$ e vamos denotar esse espaço por $G([a, b], X)$. O espaço das funções contínuas $f:[a, b] \rightarrow X$ será denotado por $C([a, b], X)$ e é claro que $C([a, b], X) \subset G([a, b], X)$ quando consideramos a norma induzida. Denotaremos por $G^{-}([a, b], X)$ o subespaço das funções regradas $f:[a, b] \rightarrow X$ que são contínuas à esquerda e, analogamente, $G^{+}([a, b], X)$ o subespaço das funções regradas $f:[a, b] \rightarrow X$ que são contínuas à direita.

Todo conjunto finito $D=\left\{t_{0}, t_{1}, \ldots, t_{|D|}\right\} \subset[a, b]$ tal que, $a=t_{0}<t_{1}<\ldots<t_{|D|}=b$, será dito uma divisão de $[a, b]$, onde $|D|$ denota o número de subintervalos da forma $\left[t_{i-1}, t_{i}\right]$ de $[a, b]$. O conjunto das divisões de $[a, b]$ será denotado por $\mathscr{D}[a, b]$.

Uma função $f:[a, b] \rightarrow X$ será dita uma função escada, se existir uma divisão $D=\left\{t_{0}, t_{1}, \ldots, t_{|D|}\right\}$ de $[a, b]$ tal que $f$ é constante em todo intervalo aberto $\left(t_{i-1}, t_{i}\right)$, $i=1, \ldots,|D|$.

O primeiro resultado desse capítulo nos dá uma importante caraterização do espaço das funções regradas e será muito útil para a demostração de vários resultados ao longo do trabalho. A prova desse resultado é baseada no Teorema 3.1 de [21]. Veja também [17].

Teorema 1.1. Seja $f$ uma função de $[a, b]$ em X. Então as seguintes propriedades são equivalentes:

(i) $f:[a, b] \rightarrow X$ é limite uniforme de funções escadas,

(ii) $f:[a, b] \rightarrow X$ é regrada, 
(iii) Dado $\epsilon>0$, existe uma divisão $D=\left\{t_{0}, t_{1}, \ldots, t_{|D|}\right\}$ de $[a, b]$ tal que

$$
\sup \left\{\|f(t)-f(s)\|, t, s \in\left(t_{i-1}, t_{i}\right), i=1, \ldots,|D|\right\}<\epsilon .
$$

Demonstração. (i) $\Rightarrow$ (ii) Seja $t \in[a, b)$. Mostremos que $\lim _{s \rightarrow t^{+}} f(s)$ existe. O outro caso é análogo.

Sejam $\left\{t_{n}\right\}$ uma sequência em $[a, b]$ tal que $t_{n} \backslash t$, isto é, $t_{n} \geq t$ para todo $n \in \mathbb{N}$ e $t_{n}$ converge para $t$, e $\left\{f_{n}\right\}$ uma sequência de funções escadas de $[a, b]$ em $X$ tal que $f_{n} \rightarrow f$ uniformemente. Então, dado $\epsilon>0$, existe $k \in \mathbb{N}$ tal que $\left\|f(t)-f_{k}(t)\right\|<\epsilon / 4$, para todo $t \epsilon$ $[a, b]$. Além disso, como $f_{k}$ é uma função escada, existe $N_{0} \in \mathbb{N}$ tal que $\left\|f_{k}\left(t_{n}\right)-f_{k}\left(t^{+}\right)\right\|<\epsilon / 4$, para todo $n \geq N_{0}$. Portanto, se $n, m \geq N_{0}$

$$
\begin{aligned}
\left\|f\left(t_{n}\right)-f\left(t_{m}\right)\right\| & \leq\left\|f\left(t_{n}\right)-f_{k}\left(t_{n}\right)\right\|+\left\|f_{k}\left(t_{n}\right)-f_{k}\left(t^{+}\right)\right\| \\
& +\left\|f_{k}\left(t^{+}\right)-f_{k}\left(t_{m}\right)\right\|+\left\|f_{k}\left(t_{m}\right)-f\left(t_{m}\right)\right\|<\epsilon .
\end{aligned}
$$

Como $X$ é um espaço de Banach, $\lim _{s \rightarrow t^{+}} f(s)$ existe.

(ii) $\Rightarrow$ (iii) Seja $\epsilon>0$ dado. Como $f:[a, b] \rightarrow X$ é uma função regrada, para todo $t \in(a, b)$, existe $\delta_{t}>0$ tal que

$$
\sup _{w, s \in\left(t-\delta_{t}, t\right)}\|f(w)-f(s)\|<\epsilon \quad \mathrm{e} \quad \sup _{w, s \in\left(t, t+\delta_{t}\right)}\|f(w)-f(s)\|<\epsilon .
$$

Analogamente, existem $\delta_{a}, \delta_{b}>0$ tais que

$$
\sup _{w, s \in\left(a, a+\delta_{a}\right)}\|f(w)-f(s)\|<\epsilon \quad \mathrm{e} \quad \sup _{w, s \in\left(b-\delta_{b}, b\right)}\|f(w)-f(s)\|<\epsilon .
$$

O conjunto de intervalos $\left\{\left[a, a+\delta_{a}\right),\left(t-\delta_{t}, t+\delta_{t}\right),\left(b-\delta_{b}, b\right] ; t \in(a, b)\right\}$ forma uma cobertura do intervalo $[a, b]$ e, portanto, existe uma divisão $D=\left\{t_{0}, t_{1}, \ldots, t_{|D|}\right\}$ de $[a, b]$ tal que 
$\left\{\left[a, a+\delta_{a}\right),\left(t_{1}-\delta_{t_{1}}, t_{1}+\delta_{t_{1}}\right), \ldots,\left(b-\delta_{b}, b\right]\right\}$ é uma cobertura finita de $[a, b] \mathrm{e}$

$$
\sup \left\{\|f(t)-f(s)\|, t, s \in\left(t_{i-1}, t_{i}\right), i=1, \ldots,|D|\right\}<\epsilon .
$$

(iii) $\Rightarrow$ (i) Dado $n \in \mathbb{N}$, sejam $D_{n}=\left\{t_{0}, t_{1}, \ldots, t_{\left|D_{n}\right|}\right\}$ uma divisão de $[a, b]$ tal que

$$
\sup \left\{\|f(t)-f(s)\|, t, s \in\left(t_{i-1}, t_{i}\right), i=1, \ldots,\left|D_{n}\right|\right\}<\frac{1}{n}
$$

e $\tau_{i} \in\left(t_{i-1}, t_{i}\right), i=1, \ldots,\left|D_{n}\right|$.

Defina

$$
f_{n}(t)=\sum_{i=1}^{\left|D_{n}\right|} f\left(\tau_{i}\right) \chi_{\left(t_{i-1}, t_{i}\right)}(t)+\sum_{i=0}^{\left|D_{n}\right|} f\left(t_{i}\right) \chi_{t_{i}}(t)
$$

É claro que $\left\{f_{n}\right\}$ é uma sequência de funções escadas que converge uniformemente para $f$.

O corolário a seguir é uma consequência imediata do teorema que acabamos de provar.

Corolário 1.2. Seja $f:[a, b] \rightarrow X$ uma função regrada. Então, para todo $\epsilon>0$, os conjuntos

$$
\left\{t \in[a, b),\left\|f\left(t^{+}\right)-f(t)\right\| \geq \epsilon\right\} \quad e \quad\left\{t \in(a, b],\left\|f(t)-f\left(t^{-}\right)\right\| \geq \epsilon\right\}
$$

são finitos. Além disso, o conjunto dos pontos de descontinuidade da função f é enumerável.

Outra classe de funções importante para o trabalho é a das funções de variação limitada, que definiremos a seguir.

Sejam $f:[a, b] \rightarrow X$ e $D=\left\{t_{0}, t_{1}, \ldots, t_{|D|}\right\}$ uma divisão de $[a, b]$. Considere

$$
S(f, D)=\sum_{i=1}^{|D|}\left\|f\left(t_{i}\right)-f\left(t_{i-1}\right)\right\|
$$

$\mathrm{e}$

$$
\operatorname{var}_{[a, b]} f=\operatorname{var}_{a}^{b} f=\sup _{D \in \mathscr{D}[a, b]} S(f, D)
$$


em que $\operatorname{var}_{a}^{b} f$ é dita variação de $f$ em $[a, b]$.

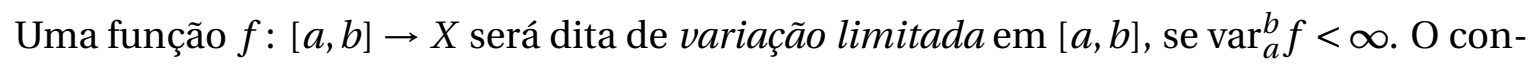
junto das funções de variação limitada de $[a, b]$ em $X$ denotaremos por $B V([a, b], X)$. Este conjunto será um espaço de Banach quando munido da norma $\|f\|_{B V}=\|f(a)\|_{X}+\operatorname{var}_{a}^{b} f$. É fácil ver que $B V([a, b], X) \subset G([a, b], X)$.

Um estudo completo sobre funções regradas pode ser encontrado em [17]. Veja também [21].

\subsection{A integral de Kurzweil em espaço de Banach}

Nesta seção, apresentaremos a definição da integral de Kurzweil e também algumas de suas propriedades. Esta integral será usada para definir equações diferenciais ordinárias generalizadas que serão introduzidas no próximo capítulo. A principal referência para esta seção é o Capítulo 1 de [33]. Veja também [23] e [24].

Iniciaremos apresentando os conceitos de divisão marcada e calibre.

Uma divisão marcada do intervalo $[a, b]$ será uma coleção finita de pontos

$$
a=t_{0} \leq \tau_{1} \leq t_{1} \leq \tau_{2} \leq t_{2} \leq \ldots \leq t_{|D|-1} \leq \tau_{|D|} \leq t_{|D|}=b
$$

em que $D=\left\{t_{0}, t_{1}, \ldots, t_{|D|}\right\}$ é uma divisão de $[a, b]$ e $\tau_{i} \in\left[t_{i-1}, t_{i}\right]$ será dito marca de $\left[t_{i-1}, t_{i}\right]$, $i=1, \ldots,|D|$. Denotaremos por $D=\left(\tau_{i}, t_{i}\right)$ ou também $D=\left(\tau_{i},\left[t_{i-1}, t_{i}\right]\right)$ uma tal divisão marcada.

Um calibre do intervalo $[a, b]$ será uma função $\delta:[a, b] \rightarrow(0, \infty)$. Dado um calibre $\delta$ de $[a, b]$, diremos que uma divisão marcada $D=\left(\tau_{i}, t_{i}\right)$ é $\delta$-fina, se

$$
\left[t_{i-1}, t_{i}\right] \subset\left(\tau_{i}-\delta\left(\tau_{i}\right), \tau_{i}+\delta\left(\tau_{i}\right)\right), \quad i=1, \ldots,|D|
$$

Através desses conceitos, podemos definir a integral de Kurzweil. 
Definição 1.3. Uma função $U:[a, b] \times[a, b] \rightarrow X$ será dita Kurzweil integrável em $[a, b]$, se existir $I \in X$ tal que dado $\epsilon>0$, existe um calibre $\delta$ de $[a, b]$ tal que para toda divisão marcada $\delta$-fina $D=\left(\tau_{i}, t_{i}\right) d e[a, b]$

$$
\|S(U, D)-I\|<\epsilon,
$$

em que $S(U, D)=\sum_{i=1}^{|D|}\left[U\left(\tau_{i}, t_{i}\right)-U\left(\tau_{i}, t_{i-1}\right)\right]$. Neste caso, I será dito a integral de Kurzweil de $U$ em $[a, b]$ e denotaremos por $I=\int_{a}^{b} D U(\tau, t)$.

Observe que se $f:[a, b] \rightarrow \mathbb{R}^{n}$ e $g:[a, b] \rightarrow \mathbb{R}$ forem funções tais que $U:[a, b] \times[a, b] \rightarrow \mathbb{R}^{n}$ é definida por $U(\tau, t)=f(\tau) g(t)$, então

$$
S(U, D)=\sum_{i=1}^{|D|}\left[U\left(\tau_{i}, t_{i}\right)-U\left(\tau_{i}, t_{i-1}\right)\right]=\sum_{i=1}^{|D|} f\left(\tau_{i}\right)\left[g\left(t_{i}\right)-g\left(t_{i-1}\right)\right]
$$

em que $S(U, D)$ representa uma soma do tipo Riemann-Stieltjes. Portanto, a integral definida acima é uma generalização da conhecida integral de Riemann-Stieltjes, isto é, se $f:[a, b] \rightarrow \mathbb{R}^{n}$ for uma função Riemann-Stieltjes integrável com respeito a $g$, então a função $U$ definida por $U(\tau, t)=f(\tau) g(t)$ será Kurzweil integrável e

$$
\int_{a}^{b} f(t) d g(t)=\int_{a}^{b} D U(\tau, t)
$$

Antes de apresentarmos algumas propriedades dessa integral, vamos ver um resultado que garante a existência de uma divisão $\delta$-fina para um calibre $\delta$ dado. Esse resultado é conhecido como Lema de Cousin e a sua prova pode ser encontra em [19], Teorema 4.1, por exemplo.

Lema 1.4 (Lema de Cousin). Dado um calibre $\delta$ de $[a, b]$, existe uma divisão marcada $\delta$-fina $d e[a, b]$.

Observação 1.5. Suponha que a integral $\int_{a}^{b} D U(\tau, t)$ exista. Então definimos

$$
\int_{b}^{a} D U(\tau, t)=-\int_{a}^{b} D U(\tau, t) .
$$


A integral de Kurzweil possui as propriedades usuais de linearidade e aditividade com respeito a intervalos adjacentes. Um importante resultado que será usado posteriormente é a integrabilidade em subintervalos (veja [33], Teorema 1.10).

Teorema 1.6. Se $U:[a, b] \times[a, b] \rightarrow X$ for Kurzweil integrável em $[a, b]$, então U será Kurzweil integrável em todo intervalo $[c, d] \subset[a, b]$.

O próximo resultado é conhecido como Lema de Saks-Henstock. Uma prova pode ser encontrada em [33], Lema 1.13, para o caso em que $\operatorname{dim} X<\infty$. No caso em que $\operatorname{dim} X=\infty$, a prova segue de modo análogo.

Lema 1.7 (Lema de Saks-Henstock). Seja $U:[a, b] \times[a, b] \rightarrow X$. Dado $\epsilon>0$, seja $\delta$ um calibre de $[a, b]$ tal que para toda divisão marcada $\delta$-fina $D=\left(\tau_{i}, t_{i}\right)$ de $[a, b]$

$$
\left\|S(U, D)-\int_{a}^{b} D U(\tau, t)\right\|<\epsilon .
$$

Se $a \leq c_{1} \leq \eta_{1} \leq d_{1} \leq c_{2} \leq \eta_{2} \leq d_{2} \leq \ldots \leq c_{m} \leq \eta_{m} \leq d_{m} \leq b$ for tal que

$$
\eta_{j} \in\left[c_{j}, d_{j}\right] \subset\left(\eta_{j}-\delta\left(\eta_{j}\right), \eta_{j}+\delta\left(\eta_{j}\right)\right), \quad j=1, \ldots, m,
$$

então

$$
\left\|\sum_{j=1}^{m}\left[U\left(\eta_{j}, d_{j}\right)-U\left(\eta_{j}, c_{j}\right)-\int_{c_{j}}^{d_{j}} D U(\tau, t)\right]\right\|<\epsilon .
$$

A seguir, veremos um importante resultado do tipo Hake (veja [33],Teorema 1.14 e Observação 1.15).

Teorema 1.8. Seja $U:[a, b] \times[a, b] \rightarrow X$.

(i) Se U for Kurzweil integrável em todo intervalo $[a, c], c \in[a, b)$ e o limite

$$
\lim _{c \rightarrow b^{-}}\left[\int_{a}^{c} D U(\tau, t)-U(b, c)+U(b, b)\right]=I \in X
$$


existir, então a função U será Kurzweil integrável em $[a, b] e$

$$
\int_{a}^{b} D U(\tau, t)=I
$$

(ii) Se U for Kurzweil integrável em todo intervalo $[c, b], c \in(a, b]$ e o limite

$$
\lim _{c \rightarrow a^{+}}\left[\int_{c}^{b} D U(\tau, t)+U(a, c)-U(a, a)\right]=I \in X
$$

existir, então a função $U$ será Kurzweil integrável em $[a, b]$ e

$$
\int_{a}^{b} D U(\tau, t)=I
$$

Esse resultado nos leva ao seguinte teorema (veja [33], Teorema 1.16).

Teorema 1.9. Se $U:[a, b] \times[a, b] \rightarrow X$ for Kurzweil integrável em $[a, b]$, então

$$
\lim _{s \rightarrow c}\left[\int_{a}^{s} D U(\tau, t)-U(c, s)+U(c, c)\right]=\int_{a}^{c} D U(\tau, t)
$$

para todo $c \in[a, b]$.

Observação 1.10. O teorema anterior nos mostra que a função definida por

$$
s \in[a, b] \mapsto \int_{a}^{s} D U(\tau, t) \in X,
$$

isto é, a integral indefinida de U não é contínua em geral. A integral indefinida será contínua em um ponto $c \in[a, b]$ se, e somente se, a função $U(c, \cdot):[a, b] \rightarrow X$ for contínua em $c$.

O último resultado desta seção é uma importante desigualdade do tipo Grownwall para a integral de Kurzweil. Este resultado pode ser visto em [33], Corolário 1.43.

Teorema 1.11. Sejam $h:[a, b] \rightarrow[0, \infty)$ uma função não decrescente e contínua à esquerda, 
$k>0$ e $l \geq 0$. Se $\psi:[a, b] \rightarrow[0, \infty)$ for uma função que satisfaz a desigualdade

$$
\psi(\xi) \leq k+l \int_{a}^{\xi} \psi(\tau) d h(\tau), \quad \xi \in[a, b]
$$

então $\psi(\xi) \leq k e^{l(h(\xi)-h(a))}$ para todo $\xi \in[a, b]$.

Vejamos, agora, um importante caso particular da integral de Kurzweil: a integral de Perron-Stieltjes.

Seja $U:[a, b] \times[a, b] \rightarrow X$ dado por $U(\tau, t)=F(t) g(\tau) \operatorname{com} F:[a, b] \rightarrow L(X)$ e $g:[a, b] \rightarrow$ $X$, em que $L(X)$ é o espaço de Banach dos operadores lineares e limitados em $X$ com a norma usual de operadores. Então a integral

$$
\int_{a}^{b} D U(\tau, t)=\int_{a}^{b} D[F(t) g(\tau)]
$$

é definida à partir de somas de Riemann da forma

$$
\sum\left[F\left(t_{i}\right)-F\left(t_{i-1}\right)\right] g\left(\tau_{i}\right)
$$

e, portanto, pode ser reescrita na forma mais convencional

$$
\int_{a}^{b} d[F(s)] g(s)
$$

a qual chamaremos de integral de Perron-Stieltjes.

A seguir, veremos alguns resultados para a integral de Perron-Stieltjes envolvendo operadores lineares. Começamos vendo uma importante estimativa para essa integral (veja [34], Proposição 10).

Proposição 1.12. Sejam $g:[a, b] \rightarrow X$ uma função regrada e $F:[a, b] \rightarrow L(X)$ uma função de 
variação limitada em $[a, b]$. Se a integral $\int_{a}^{b} d[F(s)] g(s)$ existir, então

$$
\left\|\int_{a}^{b} d[F(s)] g(s)\right\| \leq \int_{a}^{b}\|g(s)\| d\left[\operatorname{var}_{a}^{s} F\right] \leq \operatorname{var}_{a}^{b} F\|g\|
$$

Em geral, temos o seguinte resultado que garante a existência da integral de PerronStieltjes em espaços de Banach (veja [34], Proposição 15).

Teorema 1.13. Se $g:[a, b] \rightarrow X$ for uma função regrada e $F:[a, b] \rightarrow L(X)$ for uma função de variação limitada em $[a, b]$, então a integral

$$
\int_{a}^{t} d[F(s)] g(s)
$$

existirá, para todo $t \in[a, b]$.

A seguir, veremos um teorema de convergência uniforme para a integral de Perron-Stieltjes. Este resultado é um caso particular do Teorema 11 de [34].

Teorema 1.14 (Teorema da Convergência Uniforme). Sejam $F:[a, b] \rightarrow L(X)$ uma função de variação limitada em $[a, b]$ e $\left\{g_{n}\right\}$ uma sequência em $G([a, b], X)$ que converge uniformemente para um função $g \in G([a, b], X)$ em $[a, b]$. Então a integral $\int_{a}^{b} d[F(s)] g(s)$ existe e

$$
\lim _{n \rightarrow \infty} \int_{a}^{b} d[F(s)] g_{n}(s)=\int_{a}^{b} d[F(s)] g(s)
$$

Observação 1.15. No teorema anterior, podemos considerar $\left\{F_{n}\right\}_{n \in \mathbb{N}}$ uma sequência de funções de variação limitada tal que $F_{n}$ converge uniformemente para uma função $F$, também de variação limitada, que o teorema continua válido, isto é,

$$
\lim _{n \rightarrow \infty} \int_{a}^{b} d\left[F_{n}(s)\right] g_{n}(s)=\int_{a}^{b} d[F(s)] g(s)
$$

O próximo resultado é um caso particular dos Lemas 12 e 13 de [34]. Ele trata da existência da integral de Perron-Stieltjes de funções características. 
Lema 1.16. Seja $F:[a, b] \rightarrow L(X)$ uma função de variação limitada em $[a, b]$.

(a) Se $x_{0} \in X$ e c $\in[a, b]$, então

$$
\int_{a}^{b} d[F(s)] \chi_{\{c\}}(s) x_{0}= \begin{cases}\lim _{s \rightarrow a^{+}} F(s) x_{0}-F(a) x_{0}, & c=a, \\ F(b) x_{0}-\lim _{s \rightarrow b^{-}} F(s) x_{0}, & c=b, \\ \lim _{s \rightarrow c^{+}} F(s) x_{0}-\lim _{s \rightarrow c^{-}} F(s) x_{0}, & c \in(a, b) .\end{cases}
$$

(b) Se $x_{1} \in X e(c, d) \subset[a, b]$, então

$$
\int_{a}^{b} d[F(s)] \chi_{(c, d)}(s) x_{1}=\lim _{s \rightarrow d^{-}} F(s) x_{1}-\lim _{s \rightarrow c^{+}} F(s) x_{1}
$$

\subsection{A integral de Kurzweil-Cauchy em espaço de Banach}

Com a finalidade de obter alguns resultados novos e mais gerais que os encontrados na literatura, tais como uma versão do Teorema de Representação de Riesz para funções regradas e uma fórmula de variação das constantes para equações diferenciais funcionais no contexto de funções regradas, apresentamos uma integral, que chamaremos de integral de Kurzweil-Cauchy, com boas propriedades para esses fins. A grosso modo, essa integral pode ser vista como a integral de Cauchy-Stieltjes no sentido da integral de Kurzweil. Iniciaremos apresentando sua definição e veremos que essa integral satisfaz as mesmas propriedades da integral de Kurzweil apresentada na seção anterior.

Definição 1.17. Uma função $U:[a, b] \times[a, b] \rightarrow X$ será dita Kurzweil-Cauchy integrável em $[a, b]$ se existir $I \in X$ tal que dado $\epsilon>0$, existe um calibre contínuo à esquerda $\delta$ de $[a, b]$ tal que, para toda divisão marcada $\delta$-fina $D$ de $[a, b]$, vale

$$
\|S(U, D)-I\|<\epsilon .
$$

Neste caso, I será dito a integral de Kurzweil-Cauchy de $U$ em $[a, b]$, que denotaremos por 
$I=\int_{a}^{b} D U(\tau, t)$

Observação 1.18. Segue direto da definição que, se uma função $U:[a, b] \times[a, b] \rightarrow X$ for Kurzweil-Cauchy integrável em $[a, b]$, então U será Kurzweil integrável em $[a, b]$ e suas integrais coincidirão.

O Lema de Cousin (Lema 1.4), visto na seção anterior, garante a existência de uma divisão marcada $\delta$-fina de $[a, b]$ para um calibre $\delta$ dado. Veremos, agora, uma nova versão do Lema de Cousin que garante a existência de uma divisão marcada à esquerda (isto é, a marca de cada subintervalo da divisão é o extremo esquerdo desse intervalo ) $\delta$-fina de $[a, b]$, quando $\delta$ for uma função contínua à esquerda. Esse resultado será muito importante no decorrer do trabalho, pois ele permite que a integral de Kurzweil-Cauchy possa ser aproximada por uma soma com marcas específicas, neste caso o extremo esquerdo dos subintervalos da divisão. Isso permitirá que provemos uma versão do Teorema de Representação de Riesz para funções regradas e, também, que obtenhamos uma fórmula de variação das constantes para equações diferenciais funcionais lineares perturbadas, através de um teorema de correspondência.

A versão do Lema de Cousin que apresentamos a seguir é nova e sua demonstração segue os mesmos passos da demonstração do Teorema 4.1 em [19].

Lema 1.19 (Lema de Cousin). Dado um calibre contínuo à esquerda $\delta$ de $[a, b]$, existe uma divisão marcada à esquerda $\delta$-fina de $[a, b]$.

Demonstração. Suponha que o resultado seja falso. Então, se $c$ for o centro do intervalo $[a, b]$, o resultado também será falso para pelo menos um dos intervalos $[a, c]$ ou $[c, b]$. Repetindo esse processo, podemos encontramos uma sequência de intervalos fechados $I_{1} \supset I_{2} \supset I_{3} \ldots$ tal que $I_{j}$ não possui uma divisão marcada à esquerda $\delta$-fina, para todo $j=1,2, \ldots$

Sejam $I_{j}=\left[x_{j}, y_{j}\right], \operatorname{com} j=1,2, \ldots$, e $x_{0}=\bigcap_{j} I_{j}$. Como $\delta\left(x_{0}\right)>0$, existe $k \in \mathbb{N}$ tal que 
$I_{j} \subset\left(x_{0}-\delta\left(x_{0}\right), x_{0}+\delta\left(x_{0}\right)\right)$, para todo $j \geq k$, isto é

$$
x_{0}-\delta\left(x_{0}\right)<x_{j}<y_{j}<x_{0}+\delta\left(x_{0}\right), \quad j \geq k
$$

Como $x_{j} \nearrow x_{0}, y_{j} \backslash x_{0}$, isto é, $x_{j} \leq x_{0}$ e $y_{j} \geq x_{0}$ para todo $j \in \mathbb{N}, x_{j}$ e $y_{j}$ convergem para $x_{0}$, e $\delta$ é contínua à esquerda, existe $k_{1} \in \mathbb{N}$ tal que

$$
x_{j}-\delta\left(x_{j}\right)<x_{j}<y_{j}<x_{j}+\delta\left(x_{j}\right), \quad j \geq k_{1}
$$

o que é um absurdo, pois contradiz o fato de $I_{j}$ não possuir uma divisão marcada à esquerda $\delta$-fina.

Os resultados seguintes são novos e decorrentes da definição apresentada acima. As demonstrações seguem os passos das demonstração do Capítulo 1 de [33]. Faremos algumas delas nesta seção. O primeiro resultado é um critério de Cauchy para funções KurzweilCauchy integráveis.

Teorema 1.20. Um função $U:[a, b] \times[a, b] \rightarrow X$ será Kurzweil-Cauchy integrável se, e somente se, dado $\epsilon>0$, existir um calibre contínuo à esquerda $\delta$ de $[a, b]$ tal que

$$
\left\|S\left(U, D_{1}\right)-S\left(U, D_{2}\right)\right\|<\epsilon
$$

para quaisquer divisões marcadas $\delta$-finas $D_{1}, D_{2}$ de $[a, b]$.

Demonstração. Suponha que dado $n \in \mathbb{N}$, existe um calibre contínuo à esquerda $\delta$ de $[a, b]$ tal que

$$
\left\|S\left(U, D_{1}\right)-S\left(U, D_{2}\right)\right\|<1 / n
$$

para quaisquer divisões marcadas $\delta$-finas $D_{1}, D_{2}$ de $[a, b]$ e $n \in \mathbb{N}$. Para todo $n \in \mathbb{N}$, denote por $M(n)$ o conjuntos de todas as somas $S(U, D)$ que satisfazem (1.1). Note que, pelo Lema de Cousin, $M(n)$ é não vazio, para todo $n \in \mathbb{N}$. 
Seja

$$
I=\bigcap_{n \in \mathbb{N}} \overline{M(n)} \in X
$$

Portanto,

$$
\|S(U, D)-I\|<1 / n
$$

para toda divisão marcada $\delta$-fina $D$ de $[a, b]$ e para todo $n \in \mathbb{N}$. Daí, pela Definição $1.17, U$ é Kurzweil-Cauchy integrável e

$$
\int_{a}^{b} D U(\tau, t)=I
$$

Por outro lado, se $U:[a, b] \times[a, b] \rightarrow X$ for Kurzweil-Cauchy integrável, então dado $\epsilon>0$, existirá um calibre contínuo à esquerda $\delta$ de $[a, b]$ tal que, para toda divisão marcada $D$ de $[a, b]$, vale

$$
\left\|S(U, D)-\int_{a}^{b} D U(\tau, t)\right\|<\frac{\epsilon}{2}
$$

Portanto, se $D_{1}, D_{2}$ forem duas divisões marcadas $\delta$-fina de $[a, b]$, então

$$
\left\|S\left(U, D_{1}\right)-S\left(U, D_{2}\right)\right\| \leq\left\|S\left(U, D_{1}\right)-\int_{a}^{b} D U(\tau, t)\right\|+\left\|S\left(U, D_{2}\right)-\int_{a}^{b} D U(\tau, t)\right\|<\epsilon
$$

e terminamos a prova.

É claro que a integral de Kurzweil-Cauchy é linear pois, dados $c_{1}, c_{2} \in \mathbb{R}$, $U, V:[a, b] \times[a, b] \rightarrow X$ e uma divisão marcada $D$ de $[a, b]$, é fácil ver que

$$
S\left(c_{1} U+c_{2} V, D\right)=c_{1} S(U, D)+c_{2} S(V, D) \text {. }
$$

Vejamos mais alguns resultados, começando pela integrabilidade em subintervalos.

Teorema 1.21. Se $U:[a, b] \times[a, b] \rightarrow X$ for Kurzweil-Cauchy integrável em $[a, b]$, então U será Kurzweil-Cauchy integrável em todo intervalo $[c, d] \subset[a, b]$.

Demonstração. Seja $\epsilon>0$ dado. Pelo teorema anterior, existe um calibre contínuo à es- 
querda $\delta$ de $[a, b]$ tal que

$$
\left\|S\left(U, D_{1}\right)-S\left(U, D_{2}\right)\right\|<\epsilon
$$

para quaisquer divisões marcadas $\delta$-finas $D_{1}, D_{2}$ de $[a, b]$.

Suponha que $a<c<d<b$ e sejam $\widetilde{D_{1}}, \widetilde{D_{2}}$ divisões marcadas $\delta$-finas de $[c, d]$. Sejam, também, $D_{a}$ e $D_{b}$ divisões marcadas $\delta$-finas de $[a, c]$ e $[c, d]$ respectivamente. Sejam $D_{1}=D_{a} \cup \widetilde{D_{1}} \cup D_{b}$ e $D_{2}=D_{a} \cup \widetilde{D_{2}} \cup D_{b}$. É claro que $D_{1}$ e $D_{2}$ são divisões marcadas $\delta$-finas de $[a, b] \mathrm{e}$

$$
\left\|S\left(U, \widetilde{D_{1}}\right)-S\left(U, \widetilde{D_{2}}\right)\right\|=\left\|S\left(U, D_{1}\right)-S\left(U, D_{2}\right)\right\|<\epsilon,
$$

o que conclui a prova.

Teorema 1.22. Sejam $c \in[a, b]$ e $U:[a, b] \times[a, b] \rightarrow X$. Se $U$ for Kurzweil-Cauchy integrável em $[a, c]$ e em $[c, b]$, então U será Kurzweil-Cauchy integrável em $[a, b]$ e

$$
\int_{a}^{c} D U(\tau, t)+\int_{c}^{b} D U(\tau, t)=\int_{a}^{b} D U(\tau, t)
$$

Demonstração. Como $U$ é Kurzweil-Cauchy integrável em $[a, c]$ e em $[c, b]$, existem calibres contínuos à esquerda $\delta_{1}$ e $\delta_{2}$ tais que, para toda divisão marcada $D_{1} \delta_{1}$-fina de [ $\left.a, c\right]$ e para toda divisão marcada $D_{2} \delta_{2}$-fina de $[c, b]$

$$
\left\|S\left(U, D_{1}\right)-\int_{a}^{c} D U(\tau, t)\right\|<\frac{\epsilon}{2} \quad e \quad\left\|S\left(U, D_{2}\right)-\int_{c}^{b} D U(\tau, t)\right\|<\frac{\epsilon}{2} .
$$

Seja $\widetilde{\delta}:[a, b] \rightarrow(0, \infty)$ um calibre contínuo à esquerda tal que $\widetilde{\delta}(\tau)<\delta_{1}(\tau)$, se $\tau \in[a, c]$ e $\widetilde{\delta}(\tau)<\delta_{2}(\tau)$, se $\tau \in[c, b]$. Defina

$$
\delta(\tau)= \begin{cases}\min \{\widetilde{\delta}(\tau),|\tau-c|\}, & \tau \neq c, \\ \widetilde{\delta}(c), & \tau=c .\end{cases}
$$

Observe que $\delta:[a, b] \rightarrow(0, \infty)$ é um calibre contínuo à esquerda de $[a, b]$. Seja $D=\left(\tau_{i}, t_{i}\right)$ uma divisão marcada de $[a, b]$. Pela construção do calibre $\delta$, existe $m \in\{1, \ldots,|D|\}$ tal que 
$c=\tau_{m}$. Portanto,

$$
\begin{aligned}
S(U, D) & =\sum_{i=1}^{m-1}\left[U\left(\tau_{i}, t_{i}\right)-U\left(\tau_{i}, t_{i-1}\right)\right]+U\left(c, t_{m}\right)-U\left(c, t_{m-1}\right)+\sum_{i=m+1}^{|D|}\left[U\left(\tau_{i}, t_{i}\right)-U\left(\tau_{i}, t_{i-1}\right)\right] \\
& =\sum_{i=1}^{m-1}\left[U\left(\tau_{i}, t_{i}\right)-U\left(\tau_{i}, t_{i-1}\right)\right]+U(c, c)-U\left(c, t_{m-1}\right) \\
& +U\left(c, t_{m}\right)-U(c, c)+\sum_{i=m+1}^{|D|}\left[U\left(\tau_{i}, t_{i}\right)-U\left(\tau_{i}, t_{i-1}\right)\right] \\
& =S\left(U, D_{1}\right)+S\left(U, D_{2}\right)
\end{aligned}
$$

em que $D_{1}=\left\{a, \tau_{1}, t_{1}, \ldots, t_{m-1}, \tau_{m}=c\right\}$ e $D_{2}=\left\{c=\tau_{m}, t_{m} \ldots, t_{|D|-1}, \tau_{|D|}, t_{|D|}=b\right\}$ são divisões marcadas $\delta_{1}$-fina e $\delta_{2}$-fina de $[a, c]$ e $[c, b]$ respectivamente. Assim,

$$
\begin{aligned}
& \left\|S(U, D)-\left(\int_{a}^{c} D U(\tau, t)+\int_{c}^{b} D U(\tau, t)\right)\right\|=\left\|S\left(U, D_{1}\right)+S\left(U, D_{2}\right)-\int_{a}^{c} D U(\tau, t)-\int_{c}^{b} D U(\tau, t)\right\| \\
& \leq\left\|S\left(U, D_{1}\right)-\int_{a}^{c} D U(\tau, t)\right\|+\left\|S\left(U, D_{2}\right)-\int_{c}^{b} D U(\tau, t)\right\|<\epsilon .
\end{aligned}
$$

Pela definição da integral de Kurzweil-Cauchy, $\int_{a}^{b} D U(\tau, t)$ existe e vale

$$
\int_{a}^{c} D U(\tau, t)+\int_{c}^{b} D U(\tau, t)=\int_{a}^{b} D U(\tau, t)
$$

o que completa a demonstração.

O próximo resultado é uma versão do Lema de Saks-Henstock para a intergral de KurzweilCauchy. A prova segue os passos de [33], Lema 1.13.

Lema 1.23 (Lema de Saks-Henstock). Seja $U:[a, b] \times[a, b] \rightarrow X$. Dado $\epsilon>0$, seja $\delta$ um calibre contínuo à esquerda de $[a, b]$ tal que, para toda divisão marcada $\delta$-fina $D=\left(\tau_{i}, t_{i}\right)$ de $[a, b]$, temos

$$
\left\|S(U, D)-\int_{a}^{b} D U(\tau, t)\right\|<\epsilon
$$


Se $a \leq c_{1} \leq \xi_{1} \leq d_{1} \leq c_{2} \leq \xi_{2} \leq d_{2} \leq \ldots \leq c_{m} \leq \xi_{m} \leq d_{m} \leq b$ for tal que

$$
\left[c_{j}, d_{j}\right] \subset\left(\xi_{j}-\delta\left(\xi_{j}\right), \xi_{j}+\delta\left(\xi_{j}\right)\right), \quad j=1, \ldots, m
$$

então

$$
\left\|\sum_{j=1}^{m}\left[U\left(\xi_{j}, d_{j}\right)-U\left(\xi_{j}, c_{j}\right)-\int_{c_{j}}^{d_{j}} D U(\tau, t)\right]\right\|<\epsilon .
$$

Demonstração. Sem perda de generalidade, podemos assumir que $c_{j}<d_{j}, j=1, \ldots, m$. Denote $d_{0}=a$ e $c_{m+1}=b$. Se $d_{j}<c_{j+1}$ para algum $j=0, \ldots, m$, então $\int_{d_{j}}^{c_{j+1}} D U(\tau, t)$ existirá e, para todo $\eta>0$ dado, existirá um calibre contínuo à esquerda $\delta_{j}$ de $\left[d_{j}, c_{j+1}\right]$ tal que $\delta_{j}(\tau)<\delta(\tau), \tau \in\left[d_{j}, c_{j+1}\right]$ e, para toda divisão marcada $D_{j}$ de $\left[d_{j}, c_{j+1}\right]$,

$$
\left\|S\left(U, D_{j}\right)-\int_{d_{j}}^{c_{j+1}} D U(\tau, t)\right\|<\frac{\eta}{m+1} .
$$

Se $d_{j}=c_{j+1}$, então tomamos $S\left(U, D_{j}\right)=0$.

Observe que a expressão

$$
\sum_{j=1}^{m}\left[U\left(\xi_{j}, d_{j}\right)-U\left(\xi_{j}, c_{j}\right)\right]+\sum_{j=1}^{m} S\left(U, D_{j}\right)
$$

representa a soma de uma certa divisão marcada $\delta$-fina de $[a, b]$ e, consequentente,

$$
\left\|\sum_{j=1}^{m}\left[U\left(\xi_{j}, d_{j}\right)-U\left(\xi_{j}, c_{j}\right)\right]+\sum_{j=1}^{m} S\left(U, D_{j}\right)-\int_{a}^{b} D U(\tau, t)\right\|<\epsilon
$$

Portanto,

$$
\begin{aligned}
& \left\|\sum_{j=1}^{m}\left[U\left(\xi_{j}, d_{j}\right)-U\left(\xi_{j}, c_{j}\right)-\int_{c_{j}}^{d_{j}} D U(\tau, t)\right]\right\| \\
& \leq\left\|\sum_{j=1}^{m}\left[U\left(\xi_{j}, d_{j}\right)-U\left(\xi_{j}, c_{j}\right)\right]+\sum_{j=1}^{m} S\left(U, D_{j}\right)-\int_{a}^{b} D U(\tau, t)\right\|+\sum_{j=1}^{m}\left\|S\left(U, D_{j}\right)-\int_{d_{j}}^{c_{j+1}} D U(\tau, t)\right\| \\
& <\epsilon+\eta .
\end{aligned}
$$


Como a desigualdade vale para todo $\eta>0$ o resultado segue.

A seguir, veremos dois resultados do tipo Hake para a integral de Kurzweil-Cauchy. As demonstrações são análogas às demonstrações dos Teoremas 1.14 e 1.16 em [33].

Teorema 1.24. Seja $U:[a, b] \times[a, b] \rightarrow X$.

(i) Se U for Kurzweil-Cauchy integrável em todo intervalo $[a, c], c \in[a, b)$ e o limite

$$
\lim _{c \rightarrow b^{-}}\left[\int_{a}^{c} D U(\tau, t)-U(b, c)+U(b, b)\right]=I \in X
$$

existir, então a função U será Kurzweil-Cauchy integrável em $[a, b] e$

$$
\int_{a}^{b} D U(\tau, t)=I
$$

(ii) Se U for Kurzweil-Cauchy integrável em todo intervalo $[c, b], c \in(a, b]$ e o limite

$$
\lim _{c \rightarrow a^{+}}\left[\int_{c}^{b} D U(\tau, t)+U(a, c)-U(a, a)\right]=I \in X
$$

existir, então a função U será Kurzweil-Cauchy integrável em $[a, b]$ e

$$
\int_{a}^{b} D U(\tau, t)=I
$$

Teorema 1.25. Se $U:[a, b] \times[a, b] \rightarrow X$ for Kurzweil-Cauchy integrável em $[a, b]$, então

$$
\lim _{s \rightarrow c}\left[\int_{a}^{s} D U(\tau, t)-U(c, s)+U(c, c)\right]=\int_{a}^{c} D U(\tau, t)
$$

para todo $c \in[a, b]$.

Observação 1.26. Novamente, o teorema anterior nos diz que a integral indefinida de KurzweilCauchy de uma função $U$ será contínua em um ponto $c \in[a, b]$ se, e somente se, a função $U(c, \cdot):[a, b] \rightarrow X$ for contínua em $c$. 
Para finalizar esta seção, vamos apresentar o exemplo clássico de uma função que possui muita oscilação e que é Kurzweil integrável. Vamos ver que, de fato, tal função é KurzweilCauchy integrável.

Exemplo 1.27. Seja $f:[0,1] \rightarrow \mathbb{R}$ um função definida por

$$
f(t)= \begin{cases}2 t \operatorname{sen}\left(\frac{2}{t^{2}}\right)-\frac{2}{t} \cos \left(\frac{2}{t^{2}}\right), & t \in(0,1] \\ 0, & t=0\end{cases}
$$

Observe que $f$ não é Lebesgue integrável em $[0,1]$, pois $f$ é muito oscilante próximo de 0 e, portanto, não é absolutamente integrável em [0,1]. Como a integral de Riemann imprópria de $f$ existe, usando um teorema do tipo Hake para a integral de Kurzweil-Cauchy, o qual nos diz que a integral de Kurzweil-Cauchyé invariante por extensões de Cauchy (ou seja, a integral de Kurzweil-Cauchy contém suas integrais impróprias), a integral de Kurzweil-Cauchy de f também existe e tem o mesmo valor da integral de Riemann da função $f$.

\subsection{Teorema de Representação de Riesz}

Para enunciar uma versão do Teorema de Representação Riesz para funções regradas, precisamos encontrar o espaço dual dessas funções. Para isto, vejamos o conceito de semivariação limitada. Uma referência para esse assunto é [34].

Seja $L(X)$ o espaço Banach dos operadores lineares e limitados em $X$ com a norma usual $\|F\|_{L(X)}=\sup \{\|F(x)\|, x \in X,\|x\| \leq 1\}$.

Sejam $F:[a, b] \rightarrow L(X)$ e $D=\left\{t_{0}, t_{1}, \ldots, t_{|D|}\right\}$ uma divisão de $[a, b]$. Considere

$$
S V(F, D)=\sup \left\{\left\|\sum_{i=1}^{|D|}\left[F\left(t_{i}\right)-F\left(t_{i-1}\right)\right] x_{i}\right\|, x_{i} \in X,\left\|x_{i}\right\| \leq 1\right\}
$$

$\mathrm{e}$

$$
\text { (s) } \operatorname{var}_{[a, b]} F=(\mathrm{s}) \operatorname{var}_{a}^{b} F=\sup _{D \in \mathscr{D}[a, b]} S V(F, D)
$$


em que (s) $\operatorname{var}_{a}^{b} F$ é dita semivariação de $F$ em $[a, b]$.

Diremos que uma função $F:[a, b] \rightarrow L(X)$ é de semivariação limitada em $[a, b]$, se (s) $\operatorname{var}_{[a, b]} F<\infty$ e denotaremos o conjunto das funções de semivariação limitada de $[a, b]$ em $L(X)$ por $S V([a, b], L(X))$. Similarmente ao caso das funções de variação limitada, $S V([a, b], L(X))$ será um espaço de Banach quando munido da norma

$$
\|F\|_{S V}=\|F(a)\|_{L(X)}+(\mathrm{s}) \operatorname{var}_{a}^{b} F
$$

Mais ainda, podemos definir $\|F\|_{S V}=\|F(c)\|_{L(X)}+(\mathrm{s}) \operatorname{var}_{a}^{b} F$, para todo $c \in[a, b]$ e teremos uma norma em $S V([a, b], L(X))$, tornando-o um espaço de Banach. Claramente $B V([a, b], L(X)) \subset S V([a, b], L(X))$.

Seja $S V_{b}([a, b], L(X))$ o conjunto das funções $F:[a, b] \rightarrow L(X)$ que são de semivariação limitada em $[a, b]$ e $F(b)=0$. Então $S V_{b}([a, b], L(X))$ será um espaço de Banach, se considerarmos a norma

$$
\|F\|_{S V}=\|F(b)\|_{L(X)}+(\mathrm{s}) \operatorname{var}_{a}^{b} F=(\mathrm{s}) \operatorname{var}_{a}^{b} F .
$$

Considere, também, $G^{+}([a, b], X)$ o espaço das funções regradas $f:[a, b] \rightarrow X$ que são contínuas à direita e $L\left(G^{+}([a, b], X), X\right)$ o espaço dos operadores lineares e limitados de $G^{+}([a, b], X)$ em $X$.

Nesta seção, vamos considerar a integral de Kurzweil-Cauchy escrita na forma

$$
\int_{a}^{b} d[\alpha(t)] f(t)
$$

em que $\alpha:[a, b] \rightarrow L(X)$ e $f:[a, b] \rightarrow X$. Observe que, se $U:[a, b] \times[a, b] \rightarrow X$ for tal que $U(\tau, t)=\alpha(t) f(\tau)$, então dada uma divisão marcada $D=\left(\tau_{i}, t_{i}\right)$ de $[a, b]$, a soma $S(U, D)$ representa uma soma do tipo Riemann-Stieltjes

$$
S(U, D)=\sum_{i=1}^{|D|}\left[U\left(\tau_{i}, t_{i}\right)-U\left(\tau_{i}, t_{i-1}\right)\right]=\sum_{i=1}^{|D|}\left[\alpha\left(t_{i}\right)-\alpha\left(t_{i-1}\right)\right] f\left(\tau_{i}\right)
$$


o que nos motiva a usar essa notação mais convencional.

O resultado seguinte é um caso particular da Proposição 10 e do Teorema 11 ambos demonstrados em [34].

Proposição 1.28. Sejam $\alpha:[a, b] \rightarrow L(X)$ e $f:[a, b] \rightarrow X$.

(i) Se $\alpha \in S V_{b}([a, b], L(X))$ e $f \in G^{+}([a, b], X)$, então

$$
\left\|\int_{a}^{b} d[\alpha(t)] f(t)\right\| \leq(\mathrm{s}) \operatorname{var}_{a}^{b} \alpha\|f\|
$$

(ii) Sejam $\alpha \in S V_{b}([a, b], L(X)), f \in G^{+}([a, b], X)$ e $f_{n}$ uma sequência de funções em $G^{+}([a, b], X)$ que converge uniformemente para $f$. Então

$$
\int_{a}^{b} d[\alpha(t)] f(t)=\lim _{n \rightarrow \infty} \int_{a}^{b} d[\alpha(t)] f_{n}(t)
$$

O próximo lema é uma consequência dos Lemas 12 e 13 em [34] para o caso da integral de Kurzweil-Cauchy.

Lema 1.29. Sejam $\alpha \in S V_{b}([a, b], L(X)), \tau \in[a, b)$ e $x \in X$. Então

$$
\int_{a}^{b} d[\alpha(t)] \chi_{[\tau, b)}(t) x=-\alpha(\tau) x
$$

em que a integral é no sentido de Kurzweil-Cauchy.

Demonstração. Sejam $\alpha \in S V_{b}([a, b], L(X)), \tau \in[a, b)$ e $x \in X$. Dado $\epsilon>0$, sejam $\delta$ um calibre contínuo à esquerda de $[a, b]$ tal que $\delta(t)<|t-\tau|$, se $t \neq \tau$ e $D=\left(t_{i-1},\left[t_{i-1}, t_{i}\right]\right)$ uma divisão marcada $\delta$-fina de $[a, b]$ tais que

$$
\left\|\sum_{i=1}^{|D|}\left[\alpha\left(t_{i}\right)-\alpha\left(t_{i-1}\right)\right] \chi_{[\tau, b)}\left(t_{i-1}\right) x-\int_{a}^{b} d[\alpha(t)] \chi_{[\tau, b)}(t) x\right\|<\epsilon
$$


Pela definição de $\delta$, existe $m \in\{1, \ldots,|D|-1\}$ tal que $\tau=t_{m}$. Assim,

$$
\sum_{i=1}^{|D|}\left[\alpha\left(t_{i}\right)-\alpha\left(t_{i-1}\right)\right] \chi_{[\tau, b)}\left(t_{i-1}\right) x=\sum_{i=m+1}^{|D|}\left[\alpha\left(t_{i}\right)-\alpha\left(t_{i-1}\right)\right] \chi_{[\tau, b)}\left(t_{i-1}\right) x=-\alpha(\tau) x
$$

e, portanto, $\int_{a}^{b} d[\alpha(t)] \chi_{[\tau, b)}(t) x=-\alpha(\tau) x$.

Agora, vamos apresentar uma versão do Teorema de Representação de Riesz no subespaço das funções regradas contínuas à direita a valores num espaço de Banach.

Teorema 1.30 (Teorema de Representação de Riesz). Um operador $F: G^{+}([a, b], X) \rightarrow X$ será um operador linear e limitado se, e somente se, existir $\alpha \in S V_{b}([a, b], L(X))$ tal que, para toda $f \in G^{+}([a, b], X)$,

$$
F(f)=\int_{a}^{b} d[\alpha(t)] f(t)
$$

em que a integral é no sentido de Kurzweil-Cauchy.

Demonstração. Primeiramente, suponha que $\alpha \in S V_{b}([a, b], L(X))$ e que

$$
F(f)=\int_{a}^{b} d[\alpha(t)] f(t)
$$

para toda $f \in G^{+}([a, b], X)$. Segue direto da definição da integral que $F$ é um operador linear e, pelo item (i) da Proposição 1.28,

$$
\|F(f)\|=\left\|\int_{a}^{b} d[\alpha(t)] f(t)\right\| \leq(\mathrm{s}) \operatorname{var}_{a}^{b} \alpha\|f\|
$$

para toda $f \in G^{+}([a, b], X)$, isto é, $F$ é um operador linear limitado.

Suponha, agora, que $F \in L\left(G^{+}([a, b], X), X\right)$. Seja $\alpha \in S V_{b}([a, b], L(X))$ definida por

$$
\alpha(t) x= \begin{cases}-F\left(\chi_{[t, b)} x\right), & t \in[a, b) \\ 0, & t=b\end{cases}
$$


para quaisquer $t \in[a, b]$ e $x \in X$. Note que $\alpha \in S V_{b}([a, b], L(X))$, pois

$$
\begin{aligned}
S V(\alpha, D) & =\sup \left\{\left\|\sum_{i=1}^{D}\left[\alpha\left(t_{i}\right)-\alpha\left(t_{i-1}\right)\right] x_{i}\right\|, x_{i} \in X,\left\|x_{i}\right\| \leq 1\right\} \\
& =\sup \left\{\left\|F\left(\sum_{i=1}^{|D|} \chi_{\left[t_{i-1}, t_{i}\right)} x_{i}\right)\right\|, x_{i} \in X,\left\|x_{i}\right\| \leq 1\right\} \leq\|F\|
\end{aligned}
$$

para toda divisão $D=\left(t_{i}\right)$ de $[a, b]$ e, portanto, (s) $\operatorname{var}_{a}^{b} \alpha<\infty$.

Para provar que $F(f)=\int_{a}^{b} d[\alpha(t)] f(t)$ para toda $f \in G^{+}([a, b], X)$, basta provar a igualdade para funções da forma $\chi_{[\tau, b)} x, \tau \in[a, b)$ e $x \in X$, pois toda função em $G^{+}([a, b], X)$ é limite uniforme de funções dessa forma (Teorema 1.1). Sejam $\tau \in[a, b)$ e $x \in X$. Logo, pelo Lema 1.29, temos

$$
\int_{a}^{b} d[\alpha(t)] \chi_{[\tau, b)}(t) x=-\alpha(\tau) x=F\left(\chi_{[\tau, b)} x\right)
$$

O resultado segue pelo Teorema da Convergência Uniforme (item (ii) da Proposição 1.28).

Em [29] (Teorema 4.1.1), podemos encontrar uma versão do Teorema de Representação de Riesz para o $G^{-}([a, b], X)$ em escalas temporais usando integral de Cauchy-Stieltjes. Uma outra referência para esse assunto é [38] (Teorema 2.4.8). 



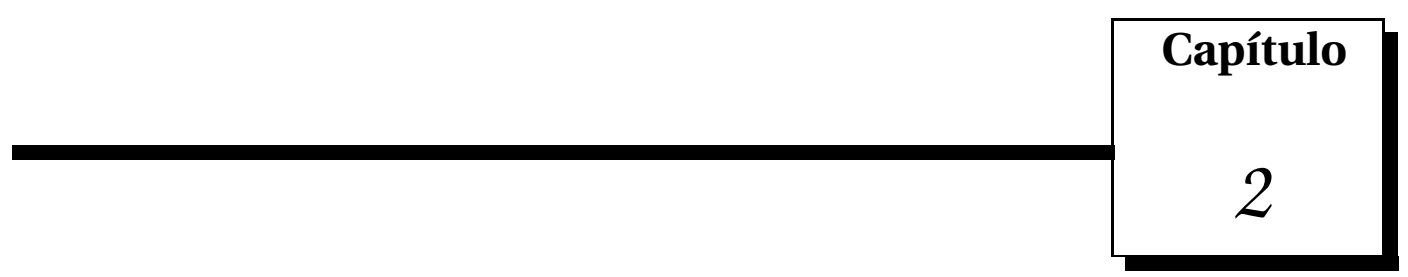

\section{EDOs generalizadas}

Neste segundo capítulo, apresentaremos as Equações Diferencias Ordinárias Generalizadas (EDOs generalizadas) em um espaço de Banach. Iniciaremos relembrando a teoria básica de EDOs generalizadas e, também, uma classe particular dessas equações, as EDOs generalizadas lineares. Na sequência, veremos a definição e algumas propriedades do operador fundamental de uma EDO generalizada linear e, finalizando o capítulo, apresentaremos alguns resultados novos, tais como uma versão da fórmula de Dirichilet para a integral de Kurzweil em espaço de Banach e uma fórmula da variação das constantes mais geral que as encontradas na literatura (veja [33], por exemplo).

\subsection{EDOs generalizadas}

Nesta seção, apresentaremos a definição de uma equação diferencial ordinária generalizada (vamos escrever EDO generalizada ou ainda EDOG) e algumas propriedades de suas soluções. Além disso, veremos um teorema de existência local e unicidade de soluções para uma certa classe de EDOs generalizadas. As principais referências para esta seção são [23], [24] e [33].

Sejam $\Omega \subset X \times \mathbb{R}$ um aberto e $G: \Omega \rightarrow X$ uma função, onde $X$ é um espaço de Banach. 
Definição 2.1. Uma função $x:[\alpha, \beta] \rightarrow X$ será dita uma solução da equação ordinária generalizada

$$
\frac{d x}{d \tau}=D G(x, t)
$$

no intervalo $[\alpha, \beta] \subset \mathbb{R}$, se $(x(t), t) \in \Omega$ para todo $t \in[\alpha, \beta]$ e se a igualdade

$$
x(v)-x(\gamma)=\int_{\gamma}^{v} D G(x(\tau), t)
$$

valer para quaisquer $\gamma, v \in[\alpha, \beta]$.

A integral do lado direito de (2.2) é no sentido da integral de Kurzweil introduzida no primeiro capítulo, Definição 1.3. A notação (2.1) é apenas simbólica. O símbolo $\frac{d x}{d \tau}$ não significa que a solução possui derivada. Vejamos um simples exemplo extraído de [33], página 100.

Exemplo 2.2. Se $r:[0,1] \rightarrow \mathbb{R}$ for uma função contínua que não tem derivada em nenhum ponto do intervalo $[0,1]$, então podemos tomar $G(x, t)=r(t)$ e, neste caso,

$$
\int_{s_{1}}^{s_{2}} \operatorname{DG}(x(\tau), t)=\int_{s_{1}}^{s_{2}} \operatorname{Dr}(t)=r\left(s_{2}\right)-r\left(s_{1}\right)
$$

Pela Definição 2.1, $x:[0,1] \rightarrow \mathbb{R}$ definida por $x(s)=r(s), s \in[0,1]$ é solução da EDO generalizada

$$
\frac{d x}{d \tau}=D G(x, t)=\operatorname{Dr}(t)
$$

e não possui derivada em nenhum ponto do intervalo $[0,1]$.

$$
\text { Sejam }-\infty<a<b<\infty \mathrm{e}
$$

$$
\Omega=O \times[a, b],
$$

em que $O \subset X$ é um conjunto aberto (por exemplo, $O=B_{c}=\{x \in X ;\|x\|<c\}$ para algum $c>0)$. 
Definição 2.3. Diremos que uma função $G: \Omega \rightarrow X$ pertence à classe $\mathscr{F}(\Omega, h)$, se existir uma função não decrescente $h:[a, b] \rightarrow \mathbb{R}$ tal que

$$
\left\|G\left(x, s_{2}\right)-G\left(x, s_{1}\right)\right\| \leq\left|h\left(s_{2}\right)-h\left(s_{1}\right)\right|
$$

para quaisquer pares $\left(x, s_{2}\right),\left(x, s_{1}\right) \in \Omega e$

$$
\left\|G\left(x, s_{2}\right)-G\left(x, s_{1}\right)-G\left(y, s_{2}\right)+G\left(y, s_{1}\right)\right\| \leq\|x-y\|\left|h\left(s_{2}\right)-h\left(s_{1}\right)\right|
$$

para quaisquer pares $\left(x, s_{2}\right),\left(x, s_{1}\right),\left(y, s_{2}\right),\left(y, s_{1}\right) \in \Omega$.

A seguir, veremos que as soluções da EDO generalizada (2.1) serão funções de variação limitada, quando $G: \Omega \rightarrow X$ pertencer à classe $\mathscr{F}(\Omega, h)$, para alguma função $h:[a, b] \rightarrow \mathbb{R}$ não decrescente. Mais geralmente, basta que $G: \Omega \rightarrow X$ satisfaça (2.3). Uma prova desse fato para $X$ com dimensão finita pode ser encontrada em [33]. A prova para o caso em que $X$ é um espaço de Banach qualquer segue de modo análogo.

Proposição 2.4. Suponha que $G: \Omega \rightarrow X$ satisfaça a condição (2.3). Se $[\alpha, \beta] \subset(a, b) e$ $x:[\alpha, \beta] \rightarrow X$ for uma solução da EDO generalizada (2.1), então para quaisquer $s_{1}, s_{2} \in[\alpha, \beta]$

$$
\left\|x\left(s_{2}\right)-x\left(s_{1}\right)\right\| \leq\left|h\left(s_{2}\right)-h\left(s_{1}\right)\right|
$$

e, consequentemente,

$$
\operatorname{var}_{\alpha}^{\beta} x \leq h(\beta)-h(\alpha)<\infty
$$

isto é, x será de variação limitada em $[\alpha, \beta]$. Além disso, se h for contínua num ponto $c \in[\alpha, \beta]$, então $x$ também será contínua em $c$.

A seguir, veremos um resultado que descreve as descontinuidades das soluções da EDO generalizada (2.1), quando a função $G$ pertence à classe $\mathscr{F}(\Omega, h)$. Este resultado pode ser encontrado em [33], Lema 3.12 para o caso em que a dimensão de $X$ é finita. O caso geral 
tem demonstração análoga.

Lema 2.5. Se $x:[\alpha, \beta] \rightarrow X$ for uma solução da EDO generalizada (2.1) $e G: \Omega \rightarrow X$ satisfizer a condição (2.3), então

$$
x(\sigma+)-x(\sigma)=\lim _{s \rightarrow \sigma+} x(s)-x(\sigma)=G(x(\sigma), \sigma+)-G(x(\sigma), \sigma)
$$

$\operatorname{para} \sigma \in[\alpha, \beta) e$

$$
x(\sigma)-x(\sigma-)=x(\sigma)-\lim _{s \rightarrow \sigma-} x(s)=G(x(\sigma), \sigma)-G(x(\sigma), \sigma-)
$$

para $\sigma \in(\alpha, \beta]$, em que

$$
G(x, \sigma+)=\lim _{s \rightarrow \sigma+} G(x, s), \quad \sigma \in[\alpha, \beta)
$$

$e$

$$
G(x, \sigma-)=\lim _{s \rightarrow \sigma-} G(x, s), \quad \sigma \in(\alpha, \beta] .
$$

Observe que os limites laterais $G(x, \sigma+), G(x, \sigma-), x(\sigma+)$ e $x(\sigma-)$ existem em $X$, pois $h$ é uma função não descrescente.

Agora apresentaremos um resultado que garante a existência da integral de Kurzweil envolvida na definição de solução da EDO generalizada (2.1). Este resultado pode ser encontrado em [1], Lema 2.7.

Lema 2.6. Sejam $G: \Omega \rightarrow X$ uma função que pertence à classe $\mathscr{F}(\Omega, h)$ e $x:[\alpha, \beta] \rightarrow X$ uma função regrada em $[\alpha, \beta] \subset[0,+\infty)$ e suponha que $(x(s), s) \in \Omega$ para todo $s \in[\alpha, \beta]$. Então a integral $\int_{\alpha}^{\beta} D G(x(\tau), t)$ existe e a função $s \mapsto \int_{\alpha}^{s} D G(x(\tau), t) \in X$ é de variação limitada em $[\alpha, \beta](e$, portanto, regrada).

O próximo resultado garante a existência e unicidade de solução para a EDO generalizada (2.1) (veja [14], Teorema 2.15). 
Teorema 2.7. Suponha que $G: \Omega \rightarrow X$ pertença à classe $\mathscr{F}(\Omega, h)$, onde a função $h$ é contínua à esquerda. Então para todo $\left(\widetilde{x}, t_{0}\right) \in \Omega$ tal que $\widetilde{x}_{+}=\widetilde{x}+G\left(\widetilde{x}, t_{0}+\right)-G\left(\widetilde{x}, t_{0}\right)$ satisfaz $\left(\widetilde{x}_{+}, t_{0}\right) \in \Omega$, existem $\Delta>0$ e uma única solução $x:\left[t_{0}, t_{0}+\Delta\right] \rightarrow X$ da EDO generalizada $(2.1)$ no intervalo $\left[t_{0}, t_{0}+\Delta\right]$ satisfazendo $x\left(t_{0}\right)=\widetilde{x}$.

Observação 2.8. A hipótese de h ser uma função contínua à esquerda no Teorema 2.7 implica que as soluções da EDO generalizada (2.1) também são contínuas à esquerda (veja equação (2.4)).

\subsection{EDOs generalizadas lineares}

Nesta seção, vamos apresentar a classe das EDOs generalizadas lineares. Veremos que, sob certas condições, podemos garantir existência global e unicidade de solução. As principais referências para esta seção são [6] e [35].

Sejam $\left(\widetilde{x}, t_{0}\right) \in X \times[a, b]$, onde $X$ é um espaço de Banach, e $L(X)$ o espaço de Banach dos operadores lineares e limitados em $X$ com a norma usual de operadores. Suponha que $F: X \times[a, b] \rightarrow X$ seja dada por $F(x, t)=A(t) x, \operatorname{com} A:[a, b] \rightarrow L(X)$ de variação limitada em $[a, b]$. Além disso, vamos supor que $A$ satisfaça as seguintes condições:

$$
\begin{aligned}
& (I+[A(t+)-A(t)])^{-1}=\left[I+\Delta^{+} A(t)\right]^{-1} \in L(X), \quad t \in[a, b) \\
& (I-[A(t)-A(t-)])^{-1}=\left[I-\Delta^{-} A(t)\right]^{-1} \in L(X), \quad t \in(a, b]
\end{aligned}
$$

em que $I \in L(X)$ é o operador identidade, $A(t+)=\lim _{s \rightarrow t+} A(s)$ e $A(t-)=\lim _{s \rightarrow t-} A(s)$.

Observação 2.9. Como $A:[a, b] \rightarrow L(X)$ é de variação limitada em $[a, b]$, os limites laterais

$$
A\left(t^{+}\right)=\lim _{r \rightarrow t^{+}} A(r) \in L(X), \quad t \in[a, b)
$$


$e$

$$
A\left(t^{-}\right)=\lim _{r \rightarrow t^{-}} A(r) \in L(X), \quad t \in(a, b]
$$

existem, pois $A \in B V([a, b], L(X)) \subset G([a, b], L(X))$. Então, pelo item (iii) doTeorema 1.1, dado $\epsilon>0$, os conjuntos

$$
\left.\left.\left\{t \in[a, b) ; \| A\left(t^{+}\right)-A(t)\right) \| \geq \epsilon\right\} \quad e \quad\left\{t \in(a, b] ; \| A(t)-A\left(t^{-}\right)\right) \| \geq \epsilon\right\}
$$

são finitos. Portanto, tomando $\epsilon=1$, existe um conjunto finito $\left\{t_{1}, t_{2}, \ldots, t_{m}\right\} \subset[a, b]$ tal que $\left.\| A\left(t^{+}\right)-A(t)\right) \|<1$ para todo $\left.t \in[a, b), t \neq t_{i}, i=1, \ldots, m, e \| A(t)-A\left(t^{-}\right)\right) \|<1$ para todo $t \in(a, b], t \neq t_{i}, i=1, \ldots, m$. Logo, os operadores

$$
I+\Delta^{+} A(t) \in L(X) \quad e \quad I-\Delta^{-} A(t) \in L(X)
$$

são invertíveis, isto é,

$$
\left[I+\Delta^{+} A(t)\right]^{-1} \in L(X), \quad t \in[a, b), t \neq t_{i}, i=1, \ldots, m,
$$

$e$

$$
\left[I-\Delta^{-} A(t)\right]^{-1} \in L(X), \quad t \in(a, b], t \neq t_{i}, i=1, \ldots, m .
$$

A observação acima nos diz que, se $A:[a, b] \rightarrow L(X)$ for um operador de variação limitada em $[a, b]$, então as condições em (2.5) são válidas a menos de uma quantidade finita de pontos em $[a, b]$.

Agora, considere o seguinte problema de valor inicial para EDO generalizada linear

$$
\left\{\begin{array}{l}
\frac{d x}{d \tau}=D F(x, t)=D[A(t) x] \\
x\left(t_{0}\right)=\widetilde{x}
\end{array}\right.
$$

Pela Definição 2.1, uma função $x:[a, b] \rightarrow X$ será uma solução da EDO generalizada linear 
(2.6) no intervalo $[a, b]$, se

$$
x\left(s_{2}\right)=x\left(s_{1}\right)+\int_{s_{1}}^{s_{2}} D[A(t) x(\tau)]
$$

para quaisquer $s_{1}, s_{2} \in[a, b]$. Em particular, uma função $x:[a, b] \rightarrow X$ será uma solução do problema de valor inicial (2.6) em $[a, b]$ se

$$
x(s)=\widetilde{x}+\int_{t_{0}}^{s} D[A(t) x(\tau)]
$$

para todo $s \in[a, b]$.

Observe que, pelas propriedades da integral de Kurzweil, a integral do lado direito de (2.7) é formada por somas de tipo Riemann-Stieltjes, isto é, as somas de Riemann para a integral $\int_{t_{0}}^{s} D[A(t) x(\tau)]$ têm a forma

$$
\sum\left[A\left(t_{i}\right)-A\left(t_{i-1}\right)\right] x\left(\tau_{i}\right)
$$

o que nos leva a uma notação mais convencional na forma $\int_{t_{0}}^{s} d[A(t)] x(t)$. Neste caso, a integral será chamada de integral de Perron-Stieltjes. Então (2.7) se torna

$$
x\left(s_{2}\right)-x\left(s_{1}\right)=\int_{s_{1}}^{s_{2}} d[A(s)] x(s), \quad s_{1}, s_{2} \in[a, b]
$$

e, similarmente, a equação (2.8) pode ser reescrita na forma

$$
x(s)=\widetilde{x}+\int_{t_{0}}^{s} d[A(r)] x(r), \quad s \in[a, b]
$$

com as integrais acima são no sentido de Perron-Stieltjes.

Observação 2.10. Quando a função $F: X \times[a, b] \rightarrow X$ tem a forma $F(x, t)=A(t) x, \operatorname{com} A(t) \epsilon$ $L(X)$ para todo $t \in[a, b]$, a integral de Kurzweil

$$
\int_{a}^{b} D F(x(\tau), t)=\int_{a}^{b} D[A(t) x(\tau)]
$$


coincide com a integral de Perron-Stieltjes, isto é,

$$
\int_{a}^{b} D F(x(\tau), t)=\int_{a}^{b} D[A(t) x(\tau)]=\int_{a}^{b} d[A(t)] x(t)
$$

Uma referência para a integral de Perron-Stieltjes, onde esta integral foi bastante estudada, é [35].

A prova do lema seguinte segue os passos da prova do Lema 6.1 em [33].

Lema 2.11. Se $x:[a, b] \rightarrow X$ for uma solução de (2.6) em $[a, b]$, então $x \in B V([a, b], X)$.

O próximo resultado garante a existência e unicidade de solução para o problema de valor inicial (2.6) no intervalo $[a, b]$ inteiro. Este resultado é consequência do Teorema 2.10 e da última observação do artigo [35].

Teorema 2.12. Se $A \in B V([a, b], L(X))$ satisfizer (2.5), então o problema de valor inicial (2.6) terá uma única solução em $[a, b]$. Além disso, essa solução será de variação limitada em $[a, b]$.

Observação 2.13. Se considerarmos $A: \mathbb{R} \rightarrow L(X)$ localmente de variação limitada, isto é, $A$ é de variação limitada em todo intervalo fechado de $\mathbb{R}$, juntamente com a hipótese (2.5), o teorema anterior garante a existência global e unicidade de solução para EDOs generalizadas lineares.

\subsection{Operador fundamental}

A fim de obter uma fórmula da variação das constantes para EDOs generalizadas, vamos definir e enunciar algumas propriedades do operador fundamental para as EDOs generalizadas lineares.

Do mesmo modo que a equação

$$
\frac{d x}{d \tau}=D[A(t) x]
$$


em (2.6), podemos considerer a equação

$$
\frac{d \Phi}{d \tau}=D[A(t) \Phi]
$$

em que $\Phi \in L(X)$. Uma solução dessa equação no intervalo $[a, b]$ é um operador $\Phi:[a, b] \rightarrow L(X)$ tal que

$$
\Phi\left(s_{2}\right)=\Phi\left(s_{1}\right)+\int_{s_{1}}^{s_{2}} D[A(t) \Phi(\tau)]=\Phi\left(s_{1}\right)+\int_{s_{1}}^{s_{2}} d[A(s)] \Phi(s)
$$

para quaisquer $s_{1}, s_{2} \in[a, b]$.

Seja $\Phi:[a, b] \rightarrow L(X)$ dado por

$$
\Phi(t)=\Phi\left(t_{0}\right)+\int_{t_{0}}^{t} d[A(s)] \Phi(s), \quad t \in[a, b]
$$

Pelo Teorema 2.12, se o operador $\Phi$ definido por (2.9) for de variação limitada em $[a, b]$ e satisfizer (2.5), então $\Phi$ será unicamente determinado. Além disso, se (2.9) for satisfeita para todo $t \in[a, b]$, então $\Phi:[a, b] \rightarrow L(X)$ será uma solução da EDO generalizada

$$
\frac{d \Phi}{d \tau}=D[A(t) \Phi]
$$

Agora, vamos definir o operador fundamental para EDOs generalizadas lineares. Veja Teorema 6.13 em [33]. Reproduzimos a prova aqui, agora para o caso de $X$ ter dimensão infinita.

Teorema 2.14. Suponha que $A \in B V([a, b], L(X))$ satisfaça (2.5). Então existe um único operador $U:[a, b] \times[a, b] \rightarrow L(X)$, chamado operador fundamental, tal que

$$
U(t, s)=I+\int_{s}^{t} d[A(r)] U(r, s)
$$

para quaisquer $t, s \in[a, b]$, onde I denota o operador identidade em $L(X)$. Além disso, para 
todo $s \in[a, b]$ fixado, $U(\cdot, s)$ será um operador de variação limitada em $[a, b]$.

Demonstração. A demonstração desse resultado segue os passos da prova do Teorema 6.13 em [33]. Dado $s \in[a, b], U(\cdot, s)$ é uma solução de

$$
\Phi(t)=I+\int_{s}^{t} d[A(r)] \Phi(r)
$$

e, pelo Teorema 2.12, esta solução existe e é de variação limitada em $[a, b]$, para todo $s \in[a, b]$ fixado.

O próximo resultado relaciona o operador fundamental da EDO generalizada com a solução do problema de valor inicial correspondente. O caso em que $\operatorname{dim} X<\infty$ pode ser encontrado em [33], Teorema 6.14.

Teorema 2.15. Suponha que $A \in B V([a, b], L(X))$ satisfaça (2.5). Então, para todo $s \in[a, b], a$ única solução $x:[a, b] \rightarrow X$ do problema de valor inicial

$$
\left\{\begin{array}{l}
\frac{d x}{d \tau}=D[A(t) x], \\
x(s)=\widetilde{x} \in X
\end{array}\right.
$$

é dada pela relação

$$
x(t)=U(t, s) \widetilde{x}, \quad t \in[a, b],
$$

em que $U:[a, b] \times[a, b] \rightarrow L(X)$ é o operador fundamental dado pelo Teorema 2.14.

Demonstração. Pelo Teorema 2.14, a função $x:[a, b] \rightarrow X$ dada por (2.12) é de variação limitada em $[a, b]$. Portanto, para todo $t \in[a, b]$, a integral $\int_{s}^{t} d[A(r)] x(r)$ existe (Teorema 1.13) e vale

$$
\int_{s}^{t} d[A(r)] x(r)=\int_{s}^{t} d[A(r)] U(r, s) \widetilde{x}=[U(t, s)-I] \widetilde{x}=x(t)-\widetilde{x}
$$

Isto significa que $x$ é uma solução do problema de valor inicial (2.11) e esta solução é unicamente determinada pelo Teorema 2.12 . 
Veremos, a seguir, um teorema com algumas propriedades do operador fundamental $U$ dado por (2.10). A prova desse resultado segue os passos da prova do Teorema $6.15 \mathrm{em}$ [33].

Teorema 2.16. Suponha que $A \in B V([a, b], L(X))$ satisfaça (2.5). Então o operador fundamental $U:[a, b] \times[a, b] \rightarrow L(X)$, dado por (2.10), satisfaz as seguintes propriedades:

(i) $U(t, t)=I$, para todo $t \in[a, b]$;

(ii) Existe uma constante $M>0$ tal que

$$
\begin{gathered}
\|U(t, s)\| \leq M, t, s \in[a, b], \\
\operatorname{var}_{a}^{b} U(t, \cdot) \leq M, t \in[a, b], \\
\operatorname{var}_{a}^{b} U(\cdot, s) \leq M, s \in[a, b] ;
\end{gathered}
$$

(iii) $U(t, s)=U(t, r) U(r, s)$, para quaisquer $t, r, s, \in[a, b]$;

(iv) $[U(t, s)]^{-1} \in L(X)$ existe e $[U(t, s)]^{-1}=U(s, t)$, para quaisquer $t, s \in[a, b]$;

(v) Valem as igualdades:

$$
\begin{aligned}
& U(t+, s)=\left[I+\Delta^{+} A(t)\right] U(t, s), \\
& U(t-, s)=\left[I-\Delta^{-} A(t)\right] U(t, s), \\
& U(t, s+)=U(t, s)\left[I+\Delta^{+} A(t)\right]^{-1}, \\
& U(t, s-)=U(t, s)\left[I-\Delta^{-} A(t)\right]^{-1},
\end{aligned}
$$

para quaisquer $t, s \in[a, b]$.

Demonstração. A propriedade (i) segue direto da definição do operador fundamental. Vamos provar a propriedade (ii). 
Sejam $s, t \in[a, b]$ fixados tais que $a \leq s<t \leq b$. Para todo $r \in[s, b]$ defina

$$
\widehat{A}(r)= \begin{cases}A(s), & r=s, \\ A\left(r^{-}\right), & r \in(s, b] .\end{cases}
$$

Como $U(\cdot, s) \in B V([a, b], L(X))$ (Teorema 2.14), valem as igualdades

$$
\begin{aligned}
\int_{s}^{t} d[A(r)] U(r, s) & =\int_{s}^{t} d[\widehat{A}(r)] U(r, s)+\left[A(t)-A\left(t^{-}\right)\right] U(t, s) \\
& =\int_{s}^{t} d[\widehat{A}(r)] U(r, s)+\Delta^{-} A(t) U(t, s)
\end{aligned}
$$

para todo $t \in(s, b]$. Logo,

$$
U(t, s)=I+\int_{s}^{t} d[A(r)] U(r, s)=I+\int_{s}^{t} d[\widehat{A}(r)] U(r, s)+\Delta^{-} A(t) U(t, s)
$$

e, assim,

$$
\left[I-\Delta^{-} A(t)\right] U(t, s)=I+\int_{s}^{t} d[A(r)] U(r, s)
$$

Portanto

$$
U(t, s)=\left[I-\Delta^{-} A(t)\right]^{-1}\left(I+\int_{s}^{t} d[A(r)] U(r, s)\right)
$$

e, pela Proposição 1.12,

$$
\|U(t, s)\| \leq\left\|\left[I-\Delta^{-} A(t)\right]^{-1}\right\|\left(1+\int_{s}^{t} d\left[\operatorname{var}_{s}^{r} \widehat{A}\right]\|U(r, s)\|\right)
$$

para todo $t \in(s, b]$.

Como $A$ satisfaz (2.5), existe uma constante $C>0$ tal que

$$
\|U(t, s)\| \leq C+C \int_{s}^{t} d\left[\operatorname{var}_{s}^{r} \widehat{A}\right]\|U(r, s)\|
$$

para todo $t \in(s, b]$. Como $\widehat{A}$ é uma função contínua à esquerda em $(s, b]$, a função $\operatorname{var}_{s}^{r} \widehat{A}$ 
também é contínua à esquerda em $(s, b]$. Portanto, pelo Teorema 1.11 (desigualdade do tipo Gronwall), obtemos

$$
\|U(t, s)\| \leq C e^{C \operatorname{var}_{s}^{t} \widehat{A}} \leq C e^{C \operatorname{var}_{a}^{b} A}:=M_{1}
$$

para quaisquer $s, t \in[a, b], \operatorname{com} s \leq t$.

Se $s, t \in[a, b]$ forem tais que $a \leq t<s$, então para todo $r \in[a, s]$ definimos

$$
\widehat{A}(r)= \begin{cases}A(s), & r=s, \\ A\left(r^{+}\right), & r \in[a, s) .\end{cases}
$$

De maneira análoga, podemos concluir que

$$
\|U(t, s)\| \leq C e^{C \operatorname{var}_{a}^{b} A},
$$

para quaisquer $s, t \in[a, b] \operatorname{com} t \leq s$. Finalmente,

$$
\|U(t, s)\| \leq M_{1}, \quad s, t \in[a, b] .
$$

Usando a estimativa obtida acima e a Proposição 1.12, obtemos

$$
\left\|U\left(t_{2}, s\right)-U\left(t_{1}, s\right)\right\|=\left\|\int_{t_{1}}^{t_{2}} d[A(r)] U(r, s)\right\| \leq M_{1} \operatorname{var}_{t_{1}}^{t_{2}} A
$$

e, portanto,

$$
\operatorname{var}_{a}^{b} U(\cdot, s) \leq M_{1} \operatorname{var}_{a}^{b} A:=M_{2},
$$

para todo $s \in[a, b]$.

Se $a \leq s_{1} \leq s_{2} \leq b$, então

$$
\begin{aligned}
U\left(t, s_{2}\right)-U\left(t, s_{1}\right) & =\int_{s_{2}}^{t} d[A(r)] U\left(r, s_{2}\right)-\int_{s_{1}}^{t} d[A(r)] U\left(r, s_{1}\right) \\
& =\int_{s_{2}}^{t} d[A(r)] U\left(r, s_{2}\right)-\int_{s_{1}}^{s_{2}} d[A(r)] U\left(r, s_{1}\right)-\int_{s_{2}}^{t} d[A(r)] U\left(r, s_{1}\right)
\end{aligned}
$$




$$
=-\int_{s_{1}}^{s_{2}} d[A(r)] U\left(r, s_{1}\right)+\int_{s_{2}}^{t} d[A(r)]\left(U\left(r, s_{2}\right)-U\left(r, s_{1}\right)\right),
$$

isto é, a função $S:[a, b] \rightarrow L(X)$, definida por $S(t)=U\left(t, s_{2}\right)-U\left(t, s_{1}\right)$, com $t \in[a, b]$, satisfaz a equação

$$
\frac{d X}{d \tau}=D[A(t) X]
$$

com condição inicial $X\left(s_{2}\right)=-\int_{s_{1}}^{s_{2}} d[A(r)] U\left(r, s_{1}\right)$. Então, pelo Teorema 2.15, temos

$$
S(t)=U\left(t, s_{2}\right)-U\left(t, s_{1}\right)=-U\left(t, s_{2}\right) \int_{s_{1}}^{s_{2}} d[A(r)] U\left(r, s_{1}\right),
$$

para todo $t \in[a, b]$. Logo,

$$
\left\|U\left(t, s_{2}\right)-U\left(t, s_{1}\right)\right\| \leq\left\|U\left(t, s_{2}\right)\right\| M_{1} \operatorname{var}_{s_{1}}^{s_{2}} A \leq M_{1}^{2} \operatorname{var}_{s_{1}}^{s_{2}} A
$$

e, portanto,

$$
\operatorname{var}_{a}^{b} U(t, \cdot) \leq M_{1}^{2} \operatorname{var}_{a}^{b} A:=M_{3}
$$

para todo $t \in[a, b]$. Tomando $M=\max \left\{M_{1}, M_{2}, M_{3}\right\}$, concluimos a prova da propriedade (ii).

Pela definição do operador fundamental $U$, é fácil ver que

$$
U(t, s)=U(r, s)+\int_{r}^{t} d[A(r)] U(r, s)
$$

para quaiquer $t, s, r \in[a, b]$ e, portanto, pelo Teorema 2.15, concluimos que

$$
U(t, s)=U(t, r) U(r, s),
$$

para quaisquer $t, s, r \in[a, b]$, isto é, a propriedade (iii) vale.

A propriedade (iv) segue direto da relação

$$
U(t, s) U(s, t)=U(t, t)=I, \quad t, s \in[a, b] .
$$


A propriedade (v) é consequência do Teorema 1.9.

\subsection{Fórmula da variação das constantes}

Para finalizar o capítulo, vamos apresentar uma fórmula da variação das constantes para EDOs generalizadas lineares perturbadas por funções que dependem do tempo e do estado. Iniciamos a seção apresentando dois lemas auxiliares para se obter uma fórmula do tipo Dirichilet que, por sua vez, será útil na obtenção da fórmula da variação das constantes. Os resultados presentes nessa seção são novos e podem ser encontrados em [6].

Lema 2.17. Sejam $t_{0} \in[a, b]$ e $U:[a, b] \times[a, b] \rightarrow L(X)$ o operador fundamental dado por (2.10) no Teorema 2.14. Então, se $\varphi \in G([a, b], X)$, a função $\widehat{\varphi}:[a, b] \rightarrow X$ dada por

$$
\widehat{\varphi}(r)=\int_{t_{0}}^{r} d_{s}[U(r, s)] \varphi(s)
$$

será regrada.

Demonstração. Sejam $t_{0} \in[a, b]$ e $\varphi \in G([a, b], X)$ fixados. Considere $r \in\left[t_{0}, b\right)$ e seja $r_{n}$ uma sequência em $[a, b]$ tal que $r_{n} \searrow r$, isto é, $r_{n} \geq r, n \in \mathbb{N}$ e $\left\{r_{n}\right\}$ converge para $r$. Pelo Teorema 2.16, $\lim _{n \rightarrow \infty} U\left(r_{n}, s\right)=U(r+, s)$ existe, para todo $s \in[a, b]$, e existe uma constante $M>0$ tal que $\operatorname{var}_{a}^{b} U\left(r_{n}, \cdot\right)<M$, para todo $n \in \mathbb{N}$. Então, pelo Teorema da Escolha de Helly ([21], Teorema I.5.8), $U(r+, \cdot) \in B V([a, b], L(X))$ e, portanto, a integral $\int_{t_{0}}^{r} d_{s}[U(r+, s)] \varphi(s)$ tem sentido.

Defina $\widetilde{U}(\sigma, s)=U(\sigma, s)$, para $a \leq s \leq \sigma \leq b$, e $\widetilde{U}(\sigma, s)=0$ para $a \leq \sigma<s \leq b$. É fácil ver que a função $\widetilde{U}$ tem as mesmas propriedades da função $U$ e, além disso, $\widetilde{U}(\sigma+, s)=U(\sigma+, s)$ para $a \leq s \leq \sigma \leq b$ e $\widetilde{U}(\sigma+, s)=0$ para $a \leq \sigma<s \leq b$. Então

$$
\begin{aligned}
\int_{t_{0}}^{b} d_{s}[\widetilde{U}(r, s)] \varphi(s) & =\int_{t_{0}}^{r} d_{s}[U(r, s)] \varphi(s)+\left[\lim _{s \rightarrow r^{+}} U(r, s)-U(r, r)\right] \varphi(r) \\
& =\widehat{\varphi}(r)+\left[\lim _{s \rightarrow r^{+}} U(r, s)-U(r, r)\right] \varphi(r) .
\end{aligned}
$$


Pela propriedade (v) no Teorema 2.16, o limite

$$
\lim _{n \rightarrow \infty}\left[\lim _{s \rightarrow r_{n}^{+}} U\left(r_{n}, s\right)-U\left(r_{n}, r_{n}\right)\right] \varphi\left(r_{n}\right)
$$

existe e, pelo Teorema da Convergênca Uniforme (Teorema 1.14), é claro que

$$
\lim _{n \rightarrow \infty} \int_{t_{0}}^{b} d_{s}\left[\widetilde{U}\left(r_{n}, s\right)-\widetilde{U}(r+, s)\right] \varphi(s)=0
$$

Então, o limite lateral à direita $\lim _{s \rightarrow r^{+}} \widehat{\varphi}(s)$ existe, para todo $r \in\left[t_{0}, t\right)$. Similarmente podemos provar que o limite lateral à esquerda $\lim _{s \rightarrow r^{-}} \widehat{\varphi}(s)$ existe, para todo $r \in\left(t_{0}, t\right]$. Portanto, a função $\widehat{\varphi}:[a, b] \rightarrow X$, dada por $\widehat{\varphi}(r)=\int_{t_{0}}^{r} d_{s}[U(r, s)] \varphi(s)$, é regrada para toda $\varphi \in$ $G([a, b], X)$.

Lema 2.18. Sejam $t_{0}, t \in[a, b], t_{0} \leq t$ e $K \in B V([a, b], L(X))$. Se $U:[a, b] \times[a, b] \rightarrow L(X)$ for o operador fundamental dado por (2.10) no Teorema 2.14, então a função $\widehat{U}:\left[t_{0}, t\right] \rightarrow L(X)$ definida por

$$
\widehat{U}(s)=\int_{s}^{t} d_{r}[K(r)] U(r, s)
$$

será de variação limitada em $\left[t_{0}, t\right]$.

Demonstração. Primeiramente, note que a propriedade (ii) no Teorema 2.16 e o fato que $K \in B V([a, b], L(X))$ implicam que a função $\widehat{U}$ está bem definida (Teorema 1.13).

Seja $\widetilde{U}(\sigma, s)=U(\sigma, s)$ para $a \leq s \leq \sigma \leq b$ e $\widetilde{U}(\sigma, s)=0$ para $a \leq \sigma<s \leq b$. É fácil ver que a função $\widetilde{U}$ tem as mesmas propriedades que a função $U$ e que, para todo $\sigma \in[a, b]$, $\operatorname{var}_{a}^{b}\left(\widetilde{U}^{\sigma}\right) \leq \operatorname{var}_{a}^{\sigma}\left(U^{\sigma}\right)+\left\|U_{\Delta}(\sigma)\right\|$, em que $U_{\Delta}(\sigma)=U(\sigma, \sigma)$. Além disso, para todo $s \in\left[t_{0}, t\right]$,

$$
\begin{aligned}
\int_{t_{0}}^{t} d[K(r)] \widetilde{U}(r, s) & =\int_{s}^{t} d[K(r)] U(r, s)+\left[K(s)-\lim _{r \rightarrow s^{-}} K(r)\right] U_{\Delta}(s) \\
& =\hat{U}(s)+\left[K(s)-\lim _{r \rightarrow s^{-}} K(r)\right] U_{\Delta}(s) .
\end{aligned}
$$


Seja $D=\left(\alpha_{j}\right), j=1, \ldots,|D|$, uma divisão de $\left[t_{0}, t\right]$. Então

$$
\begin{aligned}
& \sum_{j=1}^{|D|}\left\|\widehat{U}\left(\alpha_{j}\right)-\widehat{U}\left(\alpha_{j-1}\right)\right\| \leq \sum_{j=1}^{|D|}\left\|\int_{t_{0}}^{t} d[K(r)]\left(\widetilde{U}\left(r, \alpha_{j}\right)-\widetilde{U}\left(r, \alpha_{j-1}\right)\right)\right\| \\
& +\sum_{j=1}^{|D|}\left\|\left[K\left(\alpha_{j}\right)-\lim _{r \rightarrow \alpha_{j}^{-}} K(r)\right] U_{\Delta}\left(\alpha_{j}\right)-\left[K\left(\alpha_{j-1}\right)-\lim _{r \rightarrow \alpha_{j-1}^{-}} K(r)\right] U_{\Delta}\left(\alpha_{j-1}\right)\right\| \\
\leq & \operatorname{var}_{t_{0}}^{t}(K) \sup _{r \in\left[t_{0}, t\right]} \sum_{j=1}^{|D|}\left\|\widetilde{U}\left(r, \alpha_{j}\right)-\widetilde{U}\left(r, \alpha_{j-1}\right)\right\| \\
& +\sum_{j=1}^{|D|}\left\|\left[K\left(\alpha_{j}\right)-\lim _{r \rightarrow \alpha_{j}^{-}} K(r)\right]\left(U_{\Delta}\left(\alpha_{j}\right)-U_{\Delta}\left(\alpha_{j-1}\right)\right)\right\| \\
& +\sum_{j=1}^{|D|}\left\|\left[K\left(\alpha_{j}\right)-\lim _{r \rightarrow \alpha_{j}^{-}} K(r)-K\left(\alpha_{j-1}\right)+\lim _{r \rightarrow \alpha_{j-1}^{-}} K(r)\right] U_{\Delta}\left(\alpha_{j-1}\right)\right\| \\
\leq & \operatorname{var}_{t_{0}}^{t}(K) \sup _{r \in\left[t_{0}, t\right]} \operatorname{var}_{t_{0}}^{t} \widetilde{U}(r, \cdot)+2 \sup _{r \in\left[t_{0}, t\right]}\|K(r)\| \sum_{j=1}^{|D|}\left\|U_{\Delta}\left(\alpha_{j}\right)-U_{\Delta}\left(\alpha_{j-1}\right)\right\| \\
& +\sup _{r \in\left[t_{0}, t\right]}\left\|U_{\Delta}(r)\right\|\left(\sum_{j=1}^{|D|}\left\|K\left(\alpha_{j}\right)-K\left(\alpha_{j-1}\right)\right\|+\sum_{j=1}^{|D|}\left\|\lim _{r \rightarrow \alpha_{j}^{-}} K(r)-\lim _{r \rightarrow \alpha_{j-1}^{-}} K(r)\right\|\right) \\
\leq & \operatorname{var}_{t_{0}}^{t}(K) \sup _{r \in\left[t_{0}, t\right]}\left(\operatorname{var}_{t_{0}}^{t} U(r, \cdot)+\left\|U_{\Delta}(r)\right\|\right)+2 \sup _{r \in\left[t_{0}, t\right]}\|K(r)\| \operatorname{var}_{t_{0}}^{t}\left(U_{\Delta}\right) \\
& +2 \sup _{r \in\left[t_{0}, t\right]}\left\|U_{\Delta}(r)\right\| \operatorname{var}_{t_{0}}^{t}(K) \\
= & \operatorname{var}_{t_{0}}^{t}(K) \sup _{r \in\left[t_{0}, t\right]}\left(\operatorname{var}_{t_{0}}^{t} U(r, \cdot)+3\left\|U_{\Delta}(r)\right\|\right)+2 \sup _{r \in\left[t_{0}, t\right]}\|K(r)\| \operatorname{var}_{t_{0}}^{t}\left(U_{\Delta}\right) .
\end{aligned}
$$

Portanto $\widehat{U} \in B V\left(\left[t_{0}, t\right], L(X)\right)$ e a prova está completa.

Observação 2.19. Os dois lemas acima generalizam os Lemas 2.10 e 2.12 em [38] respectivamente, no seguinte sentido: enquanto em [38] o intervalo de integração é fixado, aqui este intervalo varia, tornando os cálculos mais difíceis de se obter. De fato, as demonstrações dos Lemas 2.17 e 2.18 vistas aqui são mais complicadas do que as demonstrações dos Lemas 2.10 e 2.12 de [38].

O próximo lema nos dá uma fórmula do tipo Dirichilet para funções regradas em espaço de Banach e será útil para provar uma fórmula da variação das constantes para EDOs generalizadas. Este lema generaliza o Lema 6.16 de [33], o qual lida com funções de variação 
limitada em $\mathbb{R}^{n}$. A técnica que vamos utilizar aqui é diferente da usada em [33]. Primeiro, calculamos as integrais de funções características e, depois, aplicamos um teorema de convergência uniforme. Em [33], o autor usa um teorema do tipo Tonelli. Em [38], também temos um resultado similar (veja [38], Teorema 2.13), mas pelas mesmas razões apresentadas na última observação, o resultado presente aqui é mais geral.

Lema 2.20. Sejam $A, K \in B V([a, b], L(X))$ e suponha que A satisfaça (2.5). Então, se $U:[a, b] \times$ $[a, b] \rightarrow L(X)$ for o operador fundamental dado por (2.10) no Teorema 2.14, a igualdade

$$
\int_{t_{0}}^{t} d[K(r)]\left(\int_{t_{0}}^{r} d_{s}[U(r, s)] \varphi(s)\right)=\int_{t_{0}}^{t} d[K(s)] \varphi(s)+\int_{t_{0}}^{t} d_{s}\left[\int_{s}^{t} d_{r}[K(r)] U(r, s)\right] \varphi(s)
$$

valerá para todo $t_{0}, t \in[a, b] e \varphi \in G([a, b], X)$.

Demonstração. Primeiramente, vamos supor que $t_{0}, t \in[a, b]$, com $t_{0} \leq t$.

Observe que, pelos Lemas 2.17 e 2.18, todas as integrais envolvidas em (2.13) estão bem definidas.

Sejam $(\alpha, \beta) \in\left[t_{0}, t\right]$ e $x_{0} \in X$ fixados. Pelo Lema 1.16, temos

$$
\int_{t_{0}}^{r} d_{s}[U(r, s)] \chi_{(\alpha, \beta)}(s) x_{o}= \begin{cases}0, & t_{0} \leq r \leq \alpha \\ x_{0}-\lim _{s \rightarrow \alpha^{+}} U(r, s) x_{0}, & \alpha<r<\beta \\ \lim _{s \rightarrow \beta^{-}} U(r, s) x_{0}-\lim _{s \rightarrow \alpha^{+}} U(r, s) x_{0}, & \beta \leq r \leq t\end{cases}
$$

Então,

$$
\begin{aligned}
& \int_{t_{0}}^{t} d[K(r)]\left(\int_{t_{0}}^{r} d_{s}[U(r, s)] \chi(\alpha, \beta)(s) x_{0}\right) \\
= & \lim _{c \rightarrow \alpha^{+}}\left(\lim _{s \rightarrow \beta^{-}} K(s) x_{0}-K(c) x_{0}+\lim _{s \rightarrow \beta^{-}}[K(\beta)-K(s)] U(\beta, s) x_{0}\right. \\
& \left.+\int_{\beta}^{t} d[K(r)]\left(\lim _{s \rightarrow \beta^{-}} U(r, s) x_{0}\right)+\int_{c}^{t} d[K(r)]\left(-\lim _{s \rightarrow \alpha^{+}} U(r, s) x_{0}\right)\right) \\
= & \lim _{s \rightarrow \beta^{-}} K(s) x_{0}-\lim _{s \rightarrow \alpha^{+}} K(s) x_{0}+\lim _{s \rightarrow \beta^{-}}[K(\beta)-K(s)] U(\beta, s) x_{0} \\
& +\int_{\beta}^{t} d[K(r)]\left(\lim _{s \rightarrow \beta^{-}} U(r, s) x_{0}\right)-\lim _{s \rightarrow \alpha^{+}} \int_{s}^{t} d[K(r)] U(r, s) x_{0} .
\end{aligned}
$$


Por outro lado,

$$
\int_{t_{0}}^{t} d[K(s)] \chi_{(\alpha, \beta)}(s) x_{0}=\lim _{s \rightarrow \beta^{-}} K(s) x_{0}-\lim _{s \rightarrow \alpha^{+}} K(s) x_{0}
$$

e, usando o Teorema 1.9, obtemos

$$
\begin{aligned}
& \int_{t_{0}}^{t} d_{s}\left[\int_{s}^{t} d_{r}[K(r)] U(r, s)\right] \chi_{(\alpha, \beta)}(s) x_{0} \\
= & \lim _{s \rightarrow \beta^{-}} \int_{s}^{t} d_{r}[K(r)] U(r, s) x_{0}-\lim _{s \rightarrow \alpha^{+}} \int_{s}^{t} d_{r}[K(r)] U(r, s) x_{0} \\
= & \lim _{s \rightarrow \beta^{-}}[K(\beta)-K(s)] U(\beta, s) x_{0}+\int_{\beta}^{t} d_{r}[K(r)]\left(\lim _{s \rightarrow \beta^{-}} U(r, s) x_{0}\right) \\
& -\lim _{s \rightarrow \alpha^{+}} \int_{s}^{t} d_{r}[K(r)] U(r, s) x_{0} .
\end{aligned}
$$

Portanto, comparando as igualdades (2.14), (2.15) e (2.16), a igualdade (2.13) vale para $\varphi(s)=$ $\chi_{(\alpha, \beta)}(s) x_{0}$.

Seja $x_{1} \in X$ e suponha $t_{0}<\gamma<t$. Usando as mesmas idéias acima, teremos

$$
\int_{t_{0}}^{r} d_{s}[U(r, s)] \chi_{\{\gamma\}}(s) x_{1}= \begin{cases}0, & t_{0} \leq r<\gamma \\ x_{1}-\lim _{s \rightarrow \gamma^{-}} U(\gamma, s) x_{1}, & r=\gamma ; \\ \lim _{s \rightarrow \gamma^{+}} U(r, s) x_{1}-\lim _{s \rightarrow \gamma^{-}} U(r, s) x_{1}, & \gamma<r \leq t\end{cases}
$$

$\mathrm{e}$

$$
\begin{aligned}
& \int_{t_{0}}^{t} d[K(r)]\left(\int_{t_{0}}^{r} d_{s}[U(r, s)] \chi_{\{\gamma\}}(s) x_{1}\right) \\
= & \int_{t_{0}}^{\gamma} d[K(r)]\left(\int_{t_{0}}^{r} d_{s}[U(r, s)] \chi_{\{\gamma\}}(s) x_{1}\right)+\int_{\gamma}^{t} d[K(r)]\left(\int_{t_{0}}^{r} d_{s}[U(r, s)] \chi_{\{\gamma\}}(s) x_{1}\right) \\
= & \lim _{s \rightarrow \gamma^{-}}[K(\gamma)-K(s)]\left(x_{1}-U(\gamma, s) x_{1}\right)-\int_{\gamma}^{t} d[K(r)]\left(\lim _{s \rightarrow \gamma^{-}} U(r, s) x_{1}\right) \\
& +\lim _{s \rightarrow \gamma^{+}}\left(\int_{s}^{t} d[K(r)]\left(\lim _{\sigma \rightarrow \gamma^{+}} U(r, \sigma) x_{1}\right)+[K(s)-K(\gamma)] x_{1}\right) \\
= & \lim _{s \rightarrow \gamma^{+}} K(s) x_{1}-\lim _{s \rightarrow \gamma^{-}} K(s) x_{1}-\lim _{s \rightarrow \gamma^{-}}[K(\gamma)-K(s)] U(\gamma, s) x_{1} \\
& +\lim _{s \rightarrow \gamma^{+}} \int_{s}^{t} d[K(r)] U(r, s) x_{1}-\int_{\gamma}^{t} d[K(r)]\left(\lim _{s \rightarrow \gamma^{-}} U(r, s) x_{1}\right)
\end{aligned}
$$


Para o lado direito de (2.13), obtemos

$$
\int_{t_{0}}^{t} d[K(s)] \chi_{\{\gamma\}}(s) x_{1}=\lim _{s \rightarrow \gamma^{+}} K(s) x_{1}-\lim _{s \rightarrow \gamma^{-}} K(s) x_{1}
$$

$\mathrm{e}$

$$
\begin{aligned}
& \int_{t_{0}}^{t} d_{s}\left[\int_{s}^{t} d[K(r)] U(r, s)\right] \chi_{\{\gamma\}}(s) x_{1} \\
= & \lim _{s \rightarrow \gamma^{+}} \int_{s}^{t} d[K(r)] U(r, s) x_{1}-\lim _{s \rightarrow \gamma^{-}} \int_{s}^{t} d[K(r)] U(r, s) x_{1} \\
= & \lim _{s \rightarrow \gamma^{+}} \int_{s}^{t} d[K(r)] U(r, s) x_{1}-\int_{\gamma}^{t} d[K(r)]\left(\lim _{s \rightarrow \gamma^{-}} U(r, s) x_{1}\right) \\
& -\lim _{s \rightarrow \gamma^{-}}[K(\gamma)-K(s)] U(\gamma, s) x_{1} .
\end{aligned}
$$

Novamente, comparando as igualdades (2.17), (2.18) e (2.19), a igualdade (2.13) vale para $\varphi(s)=\chi_{\{\gamma\}}(s) x_{1}$. Analogamente, podemos provar a igualdade (2.13) para os casos $\gamma=t_{0} \mathrm{e}$ $\gamma=t$.

Agora, sejam $\varphi \in G\left(\left[t_{0}, t\right], X\right)$ e $\varphi_{n}$ uma sequência de funções escadas que converge uniformemente para $\varphi$ em $\left[t_{0}, t\right]$, isto é

$$
\lim _{n \rightarrow \infty} \sup _{s \in\left[t_{o}, t\right]}\left\|\varphi_{n}(s)-\varphi(s)\right\|_{X}
$$

Como $\varphi_{n}$ é uma função escada para todo $n \in \mathbb{N}$, a igualdade (2.13) vale para todo $n \in \mathbb{N}$. Pelo Teorema da Convergência Uniforme para a integral de Perron (Teorema 1.14), (2.13) também vale para $\varphi$, o que conclui a demonstração.

Corolário 2.21. Suponha que $A \in B V([a, b], L(X))$ satisfaça (2.5). Então, se $U:[a, b] \times[a, b] \rightarrow$ $L(X)$ for o operador fundamental dado por (2.10) no Teorema 2.14, a igualdade

$$
\int_{t_{0}}^{t} d[A(r)]\left(\int_{t_{0}}^{r} d_{s}[U(r, s)] \varphi(s)\right)=\int_{t_{0}}^{t} d[A(s)] \varphi(s)+\int_{t_{0}}^{t} d_{s}[U(t, s)] \varphi(s)
$$

valerá para quaisquer $t_{0}, t \in[a, b]$ e $\varphi \in G([a, b], X)$. 
Demonstração. Considere $K=A$ no Lema 2.20. Então

$$
\int_{t_{0}}^{t} d[A(r)]\left(\int_{t_{0}}^{r} d_{s}[U(r, s)] \varphi(s)\right)=\int_{t_{0}}^{t} d[A(s)] \varphi(s)+\int_{t_{0}}^{t} d_{s}\left[\int_{s}^{t} d_{r}[A(r)] U(r, s)\right] \varphi(s)
$$

e, por (2.10),

$$
\int_{t_{0}}^{t} d[A(r)]\left(\int_{t_{0}}^{r} d_{s}[U(r, s)] \varphi(s)\right)=\int_{t_{0}}^{t} d[A(s)] \varphi(s)+\int_{t_{0}}^{t} d_{s}[U(t, s)] \varphi(s)
$$

para quaisquer $t_{0}, t \in[a, b]$ e $\varphi \in G([a, b], X)$.

Agora, vamos apresentar uma fórmula da variação das constantes para EDOs generalizadas lineares perturbadas por funções não necessariamente autônomas e não lineares. Este resultado é novo e generaliza o Teorema 6.17 de [33] (veja também [36], Teorema 3.4), cuja EDO generalizada envolvida é constituida de uma parte linear mais uma perturbação autônoma. A referência para esse resultado é [6].

Teorema 2.22. Sejam $A \in B V([a, b], L(X))$ e $F: X \times[a, b] \rightarrow L(X)$. Se $[\alpha, \beta] \subseteq[a, b], t_{0} \in[\alpha, \beta]$ e $x \in G([\alpha, \beta], X)$ for uma solução do problema de valor inicial

$$
\left\{\begin{array}{l}
\frac{d x}{d \tau}=D[A(t) x+F(x, t)] \\
x\left(t_{0}\right)=\widetilde{x}
\end{array}\right.
$$

então x poderá ser escrita como

$$
x(t)=U\left(t, t_{0}\right) \widetilde{x}+\int_{t_{0}}^{t} D F(x(\tau), s)-\int_{t_{0}}^{t} d_{\sigma}[U(t, \sigma)]\left(\int_{t_{0}}^{\sigma} D F(x(\tau), s)\right), \quad t \in[\alpha, \beta],
$$

em que $U:[a, b] \times[a, b] \rightarrow L(X)$ é o operador fundamental dado por (2.10).

Demonstração. Sejam $x \in G([\alpha, \beta], X)$ uma solução de (2.20) e $t_{0}, t \in[\alpha, \beta]$ tal que $t \geq t_{0}$. O caso em que $t \leq t_{0}$ pode ser provado de maneira análoga. 
Defina $\varphi(\sigma)=\int_{t_{0}}^{\sigma} D F(x(\tau), s)$, para $\sigma \in\left[t_{0}, t\right]$. Por (2.21) e (2.10), temos

$$
\begin{aligned}
\int_{t_{0}}^{t} d[A(r)] x(r)= & \int_{t_{0}}^{t} d[A(r)] U\left(r, t_{0}\right) \widetilde{x}+\int_{t_{0}}^{t} d[A(r)]\left[\int_{t_{0}}^{r} D F(x(\tau), s)\right] \\
& -\int_{t_{0}}^{t} d[A(r)]\left(\int_{t_{0}}^{r} d_{\sigma}[U(r, \sigma)]\left[\int_{t_{0}}^{\sigma} D F(x(\tau), s)\right]\right) \\
= & {\left[U\left(t, t_{0}\right)-I\right] \widetilde{x}+\int_{t_{0}}^{t} d[A(r)] \varphi(r)-\int_{t_{0}}^{t} d[A(r)]\left(\int_{t_{0}}^{r} d_{\sigma}[U(r, \sigma)] \varphi(\sigma)\right) . }
\end{aligned}
$$

e, pelo Corolário 2.21,

$$
\begin{aligned}
\int_{t_{0}}^{t} d[A(r)] x(r) & =U\left(t, t_{0}\right) \widetilde{x}-\widetilde{x}-\int_{t_{0}}^{t} d_{\sigma}[U(t, \sigma)] \phi(\sigma) \\
& =x(t)-\widetilde{x}-\int_{t_{0}}^{t} D F(x(\tau), s)
\end{aligned}
$$

uma vez que $\varphi$ é regrada. Isto conclui a prova, pois (2.22) significa que $x$, dada por (2.21), é uma solução de (2.20). 


\section{Capítulo}

\section{EDFs no contexto de EDOs generalizadas}

O objetivo desse capítulo é estabelecer uma correspondência entre as soluções de uma equação diferencial funcional linear (EDF linear) e as soluções de uma equação diferencial ordinária generalizada linear (EDO generalizada linear). Iniciaremos apresentando a construção da EDO generalizada linear a partir de uma EDF linear dada.

Recordamos que $G\left([a, b], \mathbb{R}^{n}\right)$ denota o espaço de Banach das funções regradas do intervalo fechado $[a, b]$ no espaço euclidiano $\mathbb{R}^{n}$, equipado com a norma usual do supremo. Denote por $|\cdot|$ qualquer norma em $\mathbb{R}^{n}$.

Sejam $r, \sigma>0$ e $t_{0} \in \mathbb{R}$. Dada uma função $y: \mathbb{R} \rightarrow \mathbb{R}^{n}$, seja $y_{t}:[-r, 0] \rightarrow \mathbb{R}^{n}$ definida por

$$
y_{t}(\theta)=y(t+\theta), \quad \theta \in[-r, 0],
$$

para cada $t \in \mathbb{R}$. É claro que, se $y \in G\left(\left[t_{0}-r, t_{0}+\sigma\right], \mathbb{R}^{n}\right)$, então $y_{t} \in G\left([-r, 0], \mathbb{R}^{n}\right)$ para todo $t \in\left[t_{0}, t_{0}+\sigma\right]$.

Agora, vamos considerar o seguinte problema de valor inicial para uma equação diferencial funcional linear

$$
\left\{\begin{array}{l}
\dot{y}=\mathscr{L}(t) y_{t} \\
y_{t_{0}}=\phi
\end{array}\right.
$$


em que $\phi \in G\left([-r, 0], \mathbb{R}^{n}\right)$ e $\mathscr{L}(t): G\left([-r, 0], \mathbb{R}^{n}\right) \rightarrow \mathbb{R}^{n}$ é linear e limitada para todo $t \in\left[t_{0}, t_{0}+\right.$ $\sigma]$, isto é, $\mathscr{L}(t) \in L\left(G\left([-r, 0], \mathbb{R}^{n}\right), \mathbb{R}^{n}\right)$ para todo $t \in\left[t_{0}, t_{0}+\sigma\right]$.

Iremos mostrar que uma equação diferencial funcional da forma (3.1) pode ser transformada em um equação diferencial ordinária generalizada da forma

$$
\left\{\begin{array}{l}
\frac{d x}{d \tau}=D[A(t) x] \\
x\left(t_{0}\right)=\widetilde{x}
\end{array}\right.
$$

em que $A:\left[t_{0}, t_{0}+\sigma\right] \rightarrow L\left(G\left(\left[t_{0}-r, t_{0}+\sigma\right], \mathbb{R}^{n}\right)\right), \tilde{x} \in G\left(\left[t_{0}-r, t_{0}+\sigma\right], \mathbb{R}^{n}\right)$, e cuja solução $x$ toma valores em um subespaço de $G\left(\left[t_{0}-r, t_{0}+\sigma\right], \mathbb{R}^{n}\right)$. Para esse propósito, vamos introduzir duas condições que a função $\mathscr{L}$ deve satisfazer:

(A) Para todo $y \in G\left(\left[t_{0}-r, t_{0}+\sigma\right], \mathbb{R}^{n}\right)$, a aplicação $t \mapsto \mathscr{L}(t) y_{t}$ é Kurzweil integrável em $\left[t_{0}, t_{0}+\sigma\right]$

(B) Existe uma função Lesbesgue integrável $M:\left[t_{0}-r, t_{0}+\sigma\right] \rightarrow \mathbb{R}$ tal que

$$
\left|\int_{s_{1}}^{s_{2}} \mathscr{L}(s)\left(y_{s}-z_{s}\right) d s\right| \leq \int_{s_{1}}^{s_{2}} M(s)\left\|y_{s}-z_{s}\right\| d s
$$

para quaisquer $s_{1}, s_{2} \in\left[t_{0}-r, t_{0}+\sigma\right]$ e $y, z \in G\left(\left[t_{0}, t_{0}+\sigma\right], \mathbb{R}^{n}\right)$.

Observação 3.1. A condição (B) vale na seguinte situação particular. Seja $\eta: \mathbb{R} \times \mathbb{R} \rightarrow L\left(\mathbb{R}^{n}\right)$ uma função tal que $\eta(t, \cdot)$ é contínua à esquerda e de variação limitada em $[-r, 0]$ para todo $t$ fixado. Suponha que $M(t)=\operatorname{var}_{-r}^{0} \eta(t, \cdot)$ é Lebesgue integrável em $\left[t_{0}-r, t_{0}+\sigma\right]$ e seja

$$
\mathscr{L}(t) \psi=\int_{-r}^{0} d_{\theta}[\eta(t, \theta)] \psi(\theta)
$$

Pelo Teorema 1.13, a integral de Perron-Stieltjes do lado direito de (3.3) existe para todo $t \in$ $\left[t_{0}, t_{0}+\sigma\right]$. Note que, se $t \in\left[t_{0}, t_{0}+\sigma\right]$ e $\psi \in G\left([-r, 0], \mathbb{R}^{n}\right)$, então

$$
|\mathscr{L}(t) \psi|=\left|\int_{-r}^{0} d_{\theta}[\eta(t, \theta)] \psi(\theta)\right| \leq \operatorname{var}_{-r}^{0} \eta(t, \cdot)\|\psi\|=M(t)\|\psi\|
$$


e isso, juntamente com a condição (A), implica (B).

Observação 3.2. Se considerarmos o espaço $G^{+}\left([-r, 0], \mathbb{R}^{n}\right)$ das funções regradas e contínuas à direita de $[-r, 0]$ em $\mathbb{R}^{n}$, então o Teorema de Representação de Riesz visto no primeiro capítulo (Teorema 1.30) nos dará uma representação, com a mesma forma de (3.3), para a função $\mathscr{L}(t): G\left([-r, 0], \mathbb{R}^{n}\right) \rightarrow \mathbb{R}^{n}, \operatorname{com} t \in\left[t_{0}, t_{0}+\sigma\right]$.

Seguindo os mesmos passos de [14], para $y \in G\left(\left[t_{0}-r, t_{0}+\sigma\right], \mathbb{R}^{n}\right)$ e $t \in\left[t_{0}, t_{0}+\sigma\right]$, defina

$$
F(y, t)(\vartheta)= \begin{cases}0, & t_{0}-r \leq \vartheta \leq t_{0} \\ \int_{t_{0}}^{\vartheta} \mathscr{L}(s) y_{s} d s, & t_{0} \leq \vartheta \leq t \leq t_{0}+\sigma, \\ \int_{t_{0}}^{t} \mathscr{L}(s) y_{s} d s, & t_{0} \leq t \leq \vartheta \leq t_{0}+\sigma .\end{cases}
$$

Para quaisquer $y \in G\left(\left[t_{0}-r, t_{0}+\sigma\right], \mathbb{R}^{n}\right)$ e $t \in\left[t_{0}, t_{0}+\sigma\right]$ podemos observar, olhando para (3.4), que $F(y, t)$ define uma função contínua em $\left[t_{0}-r, t_{0}+\sigma\right]$, isto é,

$$
F(y, t) \in C\left(\left[t_{0}-r, t_{0}+\sigma\right], \mathbb{R}^{n}\right) .
$$

Isto significa que, para todo $t \in\left[t_{0}, t_{0}+\sigma\right]$ fixado, um operador agindo em $G\left(\left[t_{0}-r, t_{0}+\sigma\right], \mathbb{R}^{n}\right)$ está definido. Formalmente, temos

$$
F(\cdot, t): G\left(\left[t_{0}-r, t_{0}+\sigma\right], \mathbb{R}^{n}\right) \rightarrow G\left(\left[t_{0}-r, t_{0}+\sigma\right], \mathbb{R}^{n}\right)
$$

Olhando para (3.4) podemos observar, também, que $F(y, t)$ é linear na primeira variável, isto é,

$$
F\left(\alpha y_{1}+\beta y_{2}, t\right)=\alpha F\left(y_{1}, t\right)+\beta F\left(y_{2}, t\right)
$$

para quaisquer $t \in\left[t_{0}, t_{0}+\sigma\right], \alpha, \beta \in \mathbb{R}$ e $y_{1}, y_{2} \in G\left(\left[t_{0}-r, t_{0}+\sigma\right], \mathbb{R}^{n}\right)$. Este fato segue da linearidade da integral e do operador $\mathscr{L}(t)$, com $t \in\left[t_{0}, t_{0}+\sigma\right]$. 
Reescrevendo $F$ em uma forma mais convencional, $F(y, t)=A(t) y$, teremos

$$
[A(t) y](\vartheta)= \begin{cases}0, & t_{0}-r \leq \vartheta \leq t_{0} \\ \int_{t_{0}}^{\vartheta} \mathscr{L}(s) y_{s} d s, & t_{0} \leq \vartheta \leq t \leq t_{0}+\sigma \\ \int_{t_{0}}^{t} \mathscr{L}(s) y_{s} d s, & t_{0} \leq t \leq \vartheta \leq t_{0}+\sigma .\end{cases}
$$

em que, para todo $t \in\left[t_{0}, t_{0}+\sigma\right]$ fixado, $A(t): G\left(\left[t_{0}-r, t_{0}+\sigma\right], \mathbb{R}^{n}\right) \rightarrow G\left(\left[t_{0}-r, t_{0}+\sigma\right], \mathbb{R}^{n}\right)$ é um operador linear.

Considere a norma

$$
\|A(t) y\|=\sup _{\vartheta \in\left[t_{0}-r, t_{0}+\sigma\right]}|F(y, t)(\vartheta)| .
$$

Pela definição (3.4) do operador $F$ e pelas condições (A) e (B), a estimativa

$$
|F(y, t)(\vartheta)| \leq\left|\int_{t_{0}}^{t} \mathscr{L}(s) y_{s} d s\right| \leq\|y\| \int_{t_{0}}^{t} M(s) d s
$$

é válida, para quaisquer $t \in\left[t_{0}, t_{0}+\sigma\right], \vartheta \in\left[t_{0}-r, t_{0}+\sigma\right]$ e $y \in G\left(\left[t_{0}-r, t_{0}+\sigma\right], \mathbb{R}^{n}\right)$. Então

$$
\|A(t) y\| \leq\|y\| \int_{t_{0}}^{t} M(s) d s
$$

e, portanto, o operador linear $A(t)$ é limitado para todo $t \in\left[t_{0}, t_{0}+\sigma\right]$, e assim, a aplicação

$$
A:\left[t_{0}, t_{0}+\sigma\right] \rightarrow L\left(G\left(\left[t_{0}-r, t_{0}+\sigma\right], \mathbb{R}^{n}\right)\right)
$$

satisfaz a estimativa

$$
\|A(t)\|_{L\left(G\left(\left[t_{0}-r, t_{0}+\sigma\right], \mathbb{R}^{n}\right)\right)} \leq \int_{t_{0}}^{t} M(s) d s
$$

Além disso, se $y \in G\left(\left[t_{0}-r, t_{0}+\sigma\right], \mathbb{R}^{n}\right)$ e $t_{0} \leq s_{1} \leq s_{2} \leq t_{0}+\sigma$, então (3.5) e as condições (A) e (B) implicam que 


$$
\begin{aligned}
\left\|\left[A\left(s_{2}\right)-A\left(s_{1}\right)\right] y\right\| & =\sup _{\vartheta \in\left[t_{0}-r, t_{0}+\sigma\right]}\left|\left[A\left(s_{2}\right) y\right](\vartheta)-\left[A\left(s_{1}\right) y\right](\vartheta)\right| \\
& =\sup _{\vartheta \in\left[s_{1}, s_{2}\right]}\left|\left[A\left(s_{2}\right) y\right](\vartheta)-\left[A\left(s_{1}\right) y\right](\vartheta)\right| \\
& =\sup _{\vartheta \in\left[s_{1}, s_{2}\right]}\left|\int_{s_{1}}^{\vartheta} \mathscr{L}(s) y_{s} d s\right| \\
& \leq \int_{s_{1}}^{s_{2}} M(s) d s\|y\| .
\end{aligned}
$$

Portanto,

$$
\left\|A\left(s_{2}\right)-A\left(s_{1}\right)\right\|_{L\left(G\left(\left[t_{0}-r, t_{0}+\sigma\right], \mathbb{R}^{n}\right)\right)} \leq \int_{s_{1}}^{s_{2}} M(s) d s
$$

para quaisquer $s_{1}, s_{2} \in\left[t_{0}, t_{0}+\sigma\right], s_{1} \leq s_{2}$. Disso, concluimos que

$$
A:\left[t_{0}, t_{0}+\sigma\right] \rightarrow L\left(G\left(\left[t_{0}-r, t_{0}+\sigma\right], \mathbb{R}^{n}\right)\right)
$$

é de variação limitada em $\left[t_{0}, t_{0}+\sigma\right] \mathrm{e}$

$$
\operatorname{var}_{t_{0}-r}^{t_{0}+\sigma}(A) \leq \int_{t_{0}-r}^{t_{0}+\sigma} M(s) d s
$$

Mais ainda, (3.6) implica que $A:\left[t_{0}, t_{0}+\sigma\right] \rightarrow L\left(G\left(\left[t_{0}-r, t_{0}+\sigma\right], \mathbb{R}^{n}\right)\right)$ é contínua em $\left[t_{0}, t_{0}+\sigma\right]$.

A construção do operator $A(t)$ definido por (3.5) é baseada nas ideias presentes em [31] e [22] e nos resultados apresentados em [13] e [14] que relacionam problemas de valor inicial para EDFs, em particular, problemas lineares do tipo (3.1), com uma classe de EDOs generalizadas em espaço de Banach. Vamos, no que segue, descrever esta conexão com mais detalhes.

Dado $\phi \in G\left([-r, 0], \mathbb{R}^{n}\right)$, defina

$$
x\left(t_{0}\right)(\vartheta)=\widetilde{x}(\vartheta)= \begin{cases}\phi\left(\vartheta-t_{0}\right), & t_{0}-r \leq \vartheta \leq t_{0}, \\ \phi(0)=x\left(t_{0}\right)\left(t_{0}\right), & t_{0} \leq \vartheta \leq t_{0}+\sigma .\end{cases}
$$

É fácil ver que $\widetilde{x} \in G\left(\left[t_{0}-r, t_{0}+\sigma\right], \mathbb{R}^{n}\right)$ e essa função é construída a partir da condição inicial 
do problema (3.1).

A EDO generalizada associada ao problema (3.1) é linear e tem a forma de (3.2) com espaço de fase $G\left(\left[t_{0}-r, t_{0}+\sigma\right], \mathbb{R}^{n}\right)$. A forma integral do problema (3.2) é dada por

$$
x(v)=\widetilde{x}+\int_{t_{0}}^{v} D[A(t) x(\tau)]=\widetilde{x}+\int_{t_{0}}^{v} d[A(s)] x(s)
$$

em que $v \in\left[t_{0}-r, t_{0}+\sigma\right]$ e a última integral é no sentido de Perron-Stieltjes. Por (3.6), o operador $A$ em (3.2) satisfaz as hipóteses do Teorema 2.12 e, portanto, temos existência global e unicidade de solução para o problema de valor incial (3.2).

Antes de apresentar a correspondência entre as soluções da EDF linear do tipo (3.1) e as soluções de uma certa classe de EDOs generalizadas lineares do tipo (3.2), vamos enunciar um resultado importante que pode ser encontrado em [14], Lema 3.3.

Lema 3.3. Seja $x:\left[t_{0}, t_{0}+\sigma\right] \rightarrow G\left(\left[t_{0}-r, t_{0}+\sigma\right], \mathbb{R}^{n}\right)$ a solução da EDO generalizada linear (3.2) no intervalo $\left[t_{0}, t_{0}+\sigma\right]$. Se $v \in\left[t_{0}, t_{0}+\sigma\right]$, então

$$
x(\nu)(\vartheta)=x(\nu)(v), \quad \vartheta \geq v, \vartheta \in\left[t_{0}-r, t_{0}+\sigma\right]
$$

$e$

$$
x(v)(\vartheta)=x(\vartheta)(\vartheta), \quad v \geq \vartheta, \vartheta \in\left[t_{0}-r, t_{0}+\sigma\right] .
$$

Os próximos dois teoremas relacionam as soluções da EDF linear (3.1) e da EDO generalizada linear (3.2).

Teorema 3.4. Seja $y:\left[t_{0}-r, t_{0}+\sigma\right] \rightarrow \mathbb{R}^{n}$ a solução da EDF linear (3.1) no intervalo $\left[t_{0}, t_{0}+\sigma\right]$. Dado $t \in\left[t_{0}, t_{0}+\sigma\right]$, defina

$$
x(t)(\vartheta)= \begin{cases}y(\vartheta), & \vartheta \in\left[t_{0}-r, t\right] \\ y(t), & \vartheta \in\left[t, t_{0}+\sigma\right] .\end{cases}
$$

Então $x:\left[t_{0}, t_{0}+\sigma\right] \rightarrow G\left(\left[t_{0}-r, t_{0}+\sigma\right], \mathbb{R}^{n}\right)$ definida acima é solução da EDO generalizada (3.2) 
no intervalo $\left[t_{0}, t_{0}+\sigma\right]$, com condição inicial $x\left(t_{0}\right)=\widetilde{x}$ dada por (3.7).

Demonstração. Pela Definição 2.1, precisamos mostrar que, para todo $t \in\left[t_{0}-r, t_{0}+\sigma\right]$,

$$
x(t)-x\left(t_{0}\right)=\int_{t_{0}}^{t} d[A(s)] x(s) .
$$

Sejam $\epsilon>0$ arbitrário e $t>t_{0}$ fixado. Pela continuidade de $y$ no intervalo $\left[t_{0}, t\right]$ (pois a integral indefinida de uma função Perron integrável é contínua, veja Observação 1.10), podemos tomar um calibre $\delta$ em $\left[t_{0}, t\right]$ tal que, para toda divisão $\delta$-fina $D=\left(\tau_{k}, s_{k}\right)$ de $\left[t_{0}, t\right]$,

$$
\left|y(\rho)-y\left(\tau_{k}\right)\right| \leq \epsilon, \quad \rho \in\left[\tau_{k}, \tau_{k}+\delta\left(\tau_{k}\right)\right), k=1, \ldots, m .
$$

Logo, se $D=\left(\tau_{k}, s_{k}\right)$ for uma divisão $\delta$-fina de $\left[t_{0}, t\right]$, então (3.8) implicará

$$
\left[x\left(s_{k}\right)-x\left(s_{k-1}\right)\right](\vartheta)= \begin{cases}0, & \vartheta \in\left[t_{0}-r, s_{k-1}\right] \\ \int_{s_{k-1}}^{\vartheta} \mathscr{L}(s) y_{s} d s, & \vartheta \in\left[s_{k-1}, s_{k}\right] \\ \int_{s_{k-1}}^{s_{k}} \mathscr{L}(s) y_{s} d s, & \vartheta \in\left[s_{k}, t_{0}+\sigma\right]\end{cases}
$$

e, por (3.5), teremos

$$
\left[A\left(s_{k}\right) x\left(\tau_{k}\right)-A\left(s_{k-1}\right) x\left(\tau_{k}\right)\right](\vartheta)= \begin{cases}0, & \vartheta \in\left[t_{0}-r, s_{k-1}\right] \\ \int_{s_{k-1}}^{\vartheta} \mathscr{L}(s)\left(x\left(\tau_{k}\right)_{s}\right) d s, & \vartheta \in\left[s_{k-1}, s_{k}\right] \\ \int_{s_{k-1}}^{s_{k}} \mathscr{L}(s)\left(x\left(\tau_{k}\right)_{s}\right) d s, & \vartheta \in\left[s_{k}, t_{0}+\sigma\right] .\end{cases}
$$

Portanto,

$$
\begin{aligned}
& {\left[x\left(s_{k}\right)-x\left(s_{k-1}\right)\right](\vartheta)-\left[A\left(s_{k}\right) x\left(\tau_{k}\right)-A\left(s_{k-1}\right) x\left(\tau_{k}\right)\right](\vartheta)} \\
& = \begin{cases}0, & \vartheta \in\left[t_{0}-r, s_{k-1}\right], \\
\int_{s_{k-1}}^{\vartheta} \mathscr{L}(s)\left(y_{s}-x\left(\tau_{k}\right)_{s}\right) d s, & \vartheta \in\left[s_{k-1}, s_{k}\right], \\
\int_{s_{k-1}}^{s_{k}} \mathscr{L}(s)\left(y_{s}-x\left(\tau_{k}\right)_{s}\right) d s, & \vartheta \in\left[s_{k}, t_{0}+\sigma\right] .\end{cases}
\end{aligned}
$$


e, tomando a norma do supremo, obtemos

$$
\begin{aligned}
& \left\|\left[x\left(s_{k}\right)-x\left(s_{k-1}\right)\right]-\left[A\left(s_{k}\right) x\left(\tau_{k}\right)-A\left(s_{k-1}\right) x\left(\tau_{k}\right)\right]\right\| \\
= & \sup _{\vartheta \in\left[t_{0}-r, t_{0}+\sigma\right]}\left|\left[x\left(s_{k}\right)-x\left(s_{k-1}\right)\right](\vartheta)-\left[A\left(s_{k}\right) x\left(\tau_{k}\right)-A\left(s_{k-1}\right) x\left(\tau_{k}\right)\right](\vartheta)\right| \\
= & \sup _{\vartheta \in\left[s_{k-1}, s_{k}\right]}\left|\int_{s_{k-1}}^{\vartheta} \mathscr{L}(s)\left(y_{s}-x\left(\tau_{k}\right)_{s}\right) d s\right| .
\end{aligned}
$$

Note que, se $s \leq \tau_{k}$, então $x\left(\tau_{k}\right)_{s}=y_{s} \mathrm{e}$, consequentemente,

$$
\int_{s_{k-1}}^{\vartheta} \mathscr{L}(s)\left(y_{s}-x\left(\tau_{k}\right)_{s}\right) d s= \begin{cases}0, & \vartheta \in\left[s_{k-1}, \tau_{k}\right] \\ \int_{\tau_{k}}^{\vartheta} \mathscr{L}(s)\left(y_{s}-x\left(\tau_{k}\right)_{s}\right) d s, & \vartheta \in\left[\tau_{k}, s_{k}\right]\end{cases}
$$

Então, pela condição (B), temos

$$
\left|\int_{\tau_{k}}^{\vartheta} \mathscr{L}(s)\left(y_{s}-x\left(\tau_{k}\right)_{s}\right) d s\right| \leq \int_{\tau_{k}}^{\vartheta} M(s)\left\|y_{s}-x\left(\tau_{k}\right)_{s}\right\| d s \leq \int_{\tau_{k}}^{s_{k}} M(s)\left\|y_{s}-x\left(\tau_{k}\right)_{s}\right\| d s .
$$

Se $s \in\left[\tau_{k}, s_{k}\right]$, então

$$
x\left(\tau_{k}\right)_{s}(\vartheta)=x\left(\tau_{k}\right)(s+\vartheta)= \begin{cases}y(s+\vartheta)=y_{s}(\vartheta), & s+\vartheta \leq \tau_{k} \\ y\left(\tau_{k}\right), & \tau_{k} \leq s+\vartheta\end{cases}
$$

Portanto, pela definição do calibre $\delta$, temos

$$
\left\|y_{s}-x\left(\tau_{k}\right)_{s}\right\|=\sup _{\rho \in\left[\tau_{k}, s_{k}\right]}\left|y(\rho)-y\left(\tau_{k}\right)\right| \leq \epsilon
$$

$\mathrm{e}$

$$
\int_{\tau_{k}}^{s_{k}} M(s)\left\|y_{s}-x\left(\tau_{k}\right)_{s}\right\| d s \leq \epsilon \int_{\tau_{k}}^{s_{k}} M(s) d s .
$$

Logo,

$$
\left\|x(t)-x\left(t_{0}\right)-\sum_{k=1}^{m}\left[A\left(s_{k}\right) x\left(\tau_{k}\right)-A\left(s_{k-1}\right) x\left(\tau_{k}\right)\right]\right\| \leq \epsilon \int_{t_{0}}^{t} M(s) d s .
$$


Finalmente, como $\epsilon>0$ é arbitrário, obtemos

$$
x(t)-x\left(t_{0}\right)=\int_{t_{0}}^{t} d[A(s)] x(s)
$$

para todo $t \geq t_{0}$, o que conclui da prova.

Observação 3.5. Em [14] (veja Teoremas 3.4 e 3.5) uma correspondência entre EDFs (não necessariamente lineares) e EDOs generalizadas é apresentada. Em particular, EDFs lineares podem ser vistas como EDOS generalizadas. Mas o Teorema 3.4 que acabamos de ver garante mais do que isso: que EDFs lineares podem ser tratadas como EDOs generalizadas lineares. Tal resultado é novo e pode ser encontrado em [6]. Por outro lado, como um caso particular do Teorema 3.5 de [14], uma solução de uma EDO generalizada linear pode ser vista como uma solução de uma EDF. Veremos a seguir que, na verdade, uma solução de uma EDO generalizada linear pode ser relacionada com uma solução de uma EDF linear.

O próximo resultado é um caso particular da Proposição 4.2 de [30]. A prova será repetida aqui para reforçar as técnicas utilizadas nas demonstrações dos resultados de correspondência de soluções de EDFs e EDOs generalizadas.

Teorema 3.6. Seja $x:\left[t_{0}, t_{0}+\sigma\right] \rightarrow G\left(\left[t_{0}-r, t_{0}+\sigma\right], \mathbb{R}^{n}\right)$ uma solução da EDO generalizada linear (3.2) no intervalo $\left[t_{0}, t_{0}+\sigma\right]$, com condição inicial $x\left(t_{0}\right)=\widetilde{x}$ dada por (3.7). Para todo $\vartheta \in\left[t_{0}-r, t_{0}+\sigma\right]$, defina

$$
y(\vartheta)= \begin{cases}x\left(t_{0}\right)(\vartheta), & t_{0}-r \leq \vartheta \leq t_{0} \\ x(\vartheta)(\vartheta), & t_{0} \leq \vartheta \leq t_{0}+\sigma .\end{cases}
$$

Então y: $\left[t_{0}-r, t_{0}+\sigma\right] \rightarrow \mathbb{R}^{n}$ será solução da EDF linear (3.1) no intervalo $\left[t_{0}-r, t_{0}+\sigma\right]$.

Demonstração. Primeiramente, observe que $y_{t_{o}}=\phi$, por (3.7) e (3.9). Resta-nos provar que

$$
y(\vartheta)-y\left(t_{0}\right)=\int_{t_{0}}^{\vartheta} \mathscr{L}(s) y_{s} d s
$$


para todo $\vartheta \in\left[t_{0}, t_{0}+\sigma\right]$.

Seja $\vartheta \in\left[t_{0}, t_{0}+\sigma\right]$ fixado. Pela definição de $y$ e pelo Lema 3.3, temos

$$
y(\vartheta)-y\left(t_{0}\right)=x(\vartheta)(\vartheta)-x\left(t_{0}\right)\left(t_{0}\right)=x(\vartheta)(\vartheta)-x\left(t_{0}\right)(\vartheta)=\left(\int_{t_{0}}^{\vartheta} d[A(s)] x(s)\right)(\vartheta)
$$

Seja $\epsilon>0$ fixado. Como $x:\left[t_{0}, t_{0}+\sigma\right] \rightarrow G\left(\left[t_{0}-r, t_{0}+\sigma\right], \mathbb{R}^{n}\right)$ é uma função regrada, existe uma sequência em $\left[t_{0}, \vartheta\right], t_{0}<\ldots<t_{m}=\vartheta$, tal que $\|x(t)-x(s)\|<\epsilon$, sempre que $t_{k-1}<t, s<$ $t_{k}, k=1, \ldots, m$.

Pela definição da integral de Perron-Stieltjes, $\int_{t_{0}}^{\vartheta} d[A(s)] x(s)$, seja $\delta$ um calibre em $\left[t_{0}, \vartheta\right]$ tal que

$$
\delta(\tau)<\min \left\{\frac{t_{k}-t_{k-1}}{2}, k=1, \ldots, m\right\}, \quad \tau \in\left[t, t_{0}\right]
$$

$\mathrm{e}$

$$
\delta(\tau)<\min \left\{\left|\tau-t_{k}\right|,\left|\tau-t_{k-1}\right|, \tau \in\left[t_{k-1}, t_{k}\right], k=1, \ldots, m\right\},
$$

e, além disso,

$$
\left\|\sum_{k=1}^{|D|}\left[A\left(s_{k}\right) x\left(\tau_{k}\right)-A\left(s_{k-1}\right) x\left(\tau_{k}\right)\right]-\int_{t_{0}}^{\vartheta} d[A(s)] x(s)\right\|<\epsilon,
$$

para toda divisão $\delta$-fina $D=\left(\tau_{k}, s_{k}\right) \operatorname{de}\left[t_{0}, \vartheta\right]$.

Esta escolha de $\delta$ garante que se $D=\left(\tau_{k}, s_{k}\right)$ for uma disivão $\delta$-fina de [ $\left.t_{0}, \vartheta\right]$, então cada subintervalo $\left[s_{k-1}, s_{k}\right]$ conterá no máximo um dos pontos $t_{0}, \ldots, t_{m}$ e, neste caso, $t_{k}$ será a marca correspondente deste intervalo. Mais ainda, pela continuidade da integral indefinida de Perron, podemos considerar, também, que

$$
\int_{t_{k}}^{t_{k}+\delta\left(t_{k}\right)} M(s)\left\|y_{s}-x\left(t_{k}\right)_{s}\right\| d s<\frac{\epsilon}{2 m+1}, \quad k=0, \ldots, m
$$

Se $D=\left(\tau_{k}, s_{k}\right)$ for uma divisão $\delta$-fina de [ $\left.t_{0}, \vartheta\right]$, então, por (3.10) e (3.11), teremos

$$
\left|y(\vartheta)-y\left(t_{0}\right)-\int_{t_{0}}^{\vartheta} \mathscr{L}(s) y_{s} d s\right|=\left|\left(\int_{t_{0}}^{\vartheta} d[A(s)] x(s)\right)(\vartheta)-\int_{t_{0}}^{\vartheta} \mathscr{L}(s) y_{s} d s\right|
$$




$$
\begin{aligned}
& <\epsilon+\left|\sum_{k=1}^{|D|}\left[A\left(s_{k}\right) x\left(\tau_{k}\right)-A\left(s_{k-1}\right) x\left(\tau_{k}\right)\right](\vartheta)-\int_{t_{0}}^{\vartheta} \mathscr{L}(s) y_{s} d s\right| \\
& <\epsilon+\sum_{k=1}^{|D|}\left|\left[A\left(s_{k}\right) x\left(\tau_{k}\right)-A\left(s_{k-1}\right) x\left(\tau_{k}\right)\right](\vartheta)-\int_{s_{k-1}}^{s_{k}} \mathscr{L}(s) y_{s} d s\right| .
\end{aligned}
$$

Pela definição em (3.5), vale

$$
\left[A\left(s_{k}\right) x\left(\tau_{k}\right)-A\left(s_{k-1}\right) x\left(\tau_{k}\right)\right](\vartheta)=\int_{s_{k-1}}^{s_{k}} \mathscr{L}(s)\left(x\left(\tau_{k}\right)_{s}\right) d s
$$

e isto implica que

$$
\left|\left[A\left(s_{k}\right) x\left(\tau_{k}\right)-A\left(s_{k-1}\right) x\left(\tau_{k}\right)\right](\vartheta)-\int_{s_{k-1}}^{s_{k}} \mathscr{L}(s) y_{s} d s\right|=\left|\int_{s_{k-1}}^{s_{k}} \mathscr{L}(s)\left(x\left(\tau_{k}\right)_{s}-y_{s}\right) d s\right| .
$$

Pelo Lema 3.3, se $s \in\left[s_{k-1}, \tau_{k}\right]$, então $x\left(\tau_{k}\right)_{s}=y_{s}$ e, se $s \in\left[\tau_{k}, s_{k}\right]$, então

$$
x\left(\tau_{k}\right)_{s}(\vartheta)=x\left(\tau_{k}\right)(s+\vartheta)= \begin{cases}y(s+\vartheta)=y_{s}(\vartheta), & s+\vartheta \leq \tau_{k} \\ y\left(\tau_{k}\right), & \tau_{k} \leq s+\vartheta\end{cases}
$$

Logo,

$$
\left|\int_{s_{k-1}}^{s_{k}} \mathscr{L}(s)\left(x\left(\tau_{k}\right)_{s}-y_{s}\right) d s\right|=\left|\int_{\tau_{k}}^{s_{k}} \mathscr{L}(s)\left(x\left(\tau_{k}\right)_{s}-y_{s}\right) d s\right|
$$

e, pela definição do calibre $\delta$,

$$
\left\|x\left(\tau_{k}\right)_{s}-y_{s}\right\|=\sup _{\rho \in\left[\tau_{k}, s_{k}\right]}\left|y\left(\tau_{k}\right)-y(\rho)\right| \leq \epsilon
$$

Portanto, pela condição (B), obtemos

$$
\left|\int_{\tau_{k}}^{s_{k}} \mathscr{L}(s)\left(x\left(\tau_{k}\right)_{s}-y_{s}\right) d s\right| \leq \int_{\tau_{k}}^{s_{k}} M(s)\left\|x\left(\tau_{k}\right)_{s}-y_{s}\right\| d s
$$

Note que, se a interseção de $\left[s_{k-1}, s_{k}\right]$ e $\left\{t_{1}, \ldots, t_{m}\right\}$ não for vazia, então $\tau_{k}=t_{j}$, para $j \in$ 
$\{1, \ldots, m\}$. Neste caso, teremos

$$
\int_{\tau_{k}}^{s_{k}} M(s)\left\|x\left(\tau_{k}\right)_{s}-y_{S}\right\| d s \leq \int_{t_{j}}^{t_{j}+\delta\left(t_{j}\right)} M(s)\left\|x\left(t_{j}\right)_{s}-y_{s}\right\| d s<\frac{\epsilon}{2 m+1} .
$$

Por outro lado, se a interseção de $\left[s_{k-1}, s_{k}\right]$ e $\left\{t_{1}, \ldots, t_{m}\right\}$ for vazia, então

$$
\int_{\tau_{k}}^{s_{k}} M(s)\left\|x\left(\tau_{k}\right)_{s}-y_{s}\right\| d s \leq \epsilon \int_{\tau_{k}}^{s_{k}} M(s) d s
$$

Portanto,

$$
\begin{aligned}
& \left|y(\vartheta)-y\left(t_{0}\right)-\int_{t_{0}}^{\vartheta} \mathscr{L}(s) y_{s} d s\right| \\
& <\epsilon+\sum_{k=1}^{|D|}\left|\left[A\left(s_{k}\right) x\left(\tau_{k}\right)-A\left(s_{k-1}\right) x\left(\tau_{k}\right)\right](\vartheta)-\int_{s_{k-1}}^{s_{k}} \mathscr{L}(s) y_{s} d s\right| \\
& \quad<\epsilon+\epsilon \int_{t_{0}}^{\vartheta} M(s) d s+\frac{2 m \epsilon}{2 m+1} .
\end{aligned}
$$

Por fim, como $\epsilon>0$ é arbitrário, concluimos que

$$
y(\vartheta)-y\left(t_{0}\right)=\int_{t_{0}}^{\vartheta} \mathscr{L}(s) y_{s} d s
$$

para todo $\vartheta \in\left[t_{0}-r, t_{0}+\sigma\right]$, o que finaliza a demonstração.

Os dois últimos resultados (Teoremas 3.4 e 3.6), produzem uma correspondência biunívoca entre a solução da EDF linear (3.1) e a solução da EDO generalizada linear (3.2). Então, para resultados envolvendo as soluções, é possível transferir propriedades da solução de um problema para a solução do outro problema e vice-versa. Neste trabalho, vamos usar a fórmula da variação das constantes que obtivemos para EDOs generalizadas (Teorema 2.22) para conseguir uma fórmula da variação das constantes para EDFs lineares perturbadas que generalize os resultados existentes na literatura. Vejamos essa aplicação no capítulo a seguir. 


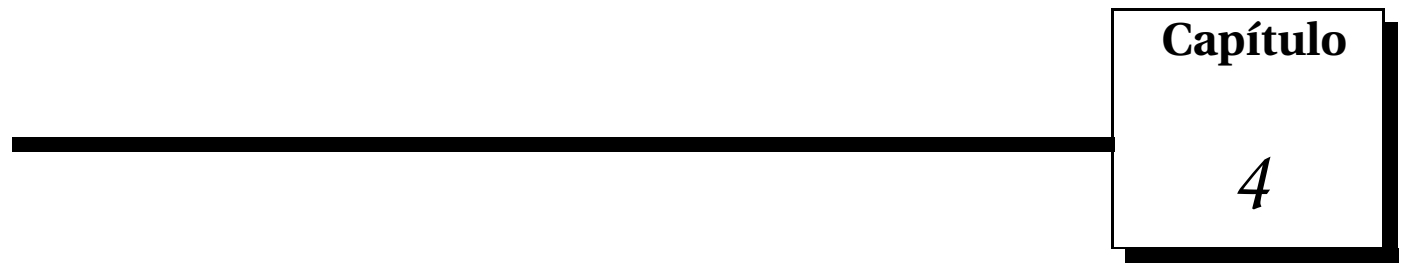

\section{Aplicações}

Neste capítulo, vamos apresentar algumas aplicações da fórmula da variação das constantes para EDOs generalizadas que obtivemos no Capítulo 2. Veremos uma fómula da variação das constantes para EDFs sob condições mais fracas que àquelas encontradas na literatura, assim como para EDFs com impulsos prefixados.

\subsection{Fórmula da variação das constantes para EDFs}

Considere a EDF linear perturbada

$$
\left\{\begin{array}{l}
\dot{y}=\mathscr{L}(t) y_{t}+f\left(y_{t}, t\right), \\
y_{t_{0}}=\phi
\end{array}\right.
$$

em que $f: G\left([-r, 0], \mathbb{R}^{n}\right) \times\left[t_{0}, t_{0}+\sigma\right] \rightarrow \mathbb{R}^{n}$ e $\mathscr{L}(t): G\left([-r, 0], \mathbb{R}^{n}\right) \rightarrow \mathbb{R}^{n}$ satisfazem as condições (A) e (B) (veja página 66), $\mathscr{L}(t)$ é um operador linear e limitado para todo $t \in\left[t_{0}, t_{0}+\sigma\right]$ e $\phi \in G\left([-r, 0], \mathbb{R}^{n}\right)$. 
Assim como em [14], definimos, para toda $y \in G\left([-r, 0], \mathbb{R}^{n}\right)$ e $t \in\left[t_{0}-r, t_{0}+\sigma\right]$,

$$
F(y, t)(\vartheta)= \begin{cases}0, & t_{0}-r \leq \vartheta \leq t_{0}, \\ \int_{t_{0}}^{\vartheta} f\left(y_{s}, s\right) d s, & t_{0} \leq \vartheta \leq t, \\ \int_{t_{0}}^{t} f\left(y_{s}, s\right) d s, & t \leq \vartheta \leq t_{0}+\sigma .\end{cases}
$$

Os Teoremas 3.4 e 3.5 em [14] e os Teoremas 3.4 e 3.6 neste trabalho implicam que existe uma correspondência biunívoca entre a equação (4.1) e a seguinte EDO generalizada

$$
\left\{\begin{array}{l}
\frac{d x}{d \tau}=D[A(t) x+F(x, t)] \\
x\left(t_{0}\right)=\widetilde{x}
\end{array}\right.
$$

em que $A:\left[t_{0}-r, t_{0}+\sigma\right] \rightarrow L\left(G\left(\left[t_{0}-r, t_{0}+\sigma\right], \mathbb{R}^{n}\right)\right)$ é dado por

$$
[A(t) y](\vartheta)= \begin{cases}0, & t_{0}-r \leq \vartheta \leq t_{0} \\ \int_{t_{0}}^{\vartheta} \mathscr{L}(s) y_{s} d s, & t_{0} \leq \vartheta \leq t \leq t_{0}+\sigma \\ \int_{t_{0}}^{t} \mathscr{L}(s) y_{s} d s, & t_{0} \leq t \leq \vartheta \leq t_{0}+\sigma\end{cases}
$$

e $\widetilde{x}$ é dado por

$$
x\left(t_{0}\right)(\vartheta)=\widetilde{x}(\vartheta)= \begin{cases}\phi\left(\vartheta-t_{0}\right), & t_{0}-r \leq \vartheta \leq t_{0}, \\ \phi(0)=x\left(t_{0}\right)\left(t_{0}\right), & t_{0} \leq \vartheta \leq t_{0}+\sigma .\end{cases}
$$

Antes de estabelecer uma fórmula da variação das constantes para a EDF linear perturbada (4.1), precisamos de alguns resultados auxiliares que apresentaremos a seguir. O primeiro resultado (Lema 4.1) relaciona as integrais da perturbação não linear dos problemas (4.1) e (4.3).

Lema 4.1. Sejam y e x soluções dos problemas perturbados (4.1) e (4.3) respectivamente. Então, para $t_{0} \leq t \leq t_{0}+\sigma$, vale 


$$
\int_{t_{0}}^{t} D F(x(\tau), s)(\vartheta)= \begin{cases}0, & t_{0}-r \leq \vartheta \leq t_{0} \\ \int_{t_{0}}^{\vartheta} f\left(y_{s}, s\right) d s, & t_{0} \leq \vartheta \leq t \\ \int_{t_{0}}^{t} f\left(y_{s}, s\right) d s, & t \leq \vartheta \leq t_{0}+\sigma .\end{cases}
$$

Demonstração. Pela definição de $F$ em (4.2), é fácil ver que $\int_{t_{0}}^{t} D F(x(\tau), s)(\vartheta)=0$, para $\vartheta \in$ $\left[t_{0}-r, t_{0}\right]$

Seja $\epsilon>0$ dado. Como $y:\left[t_{0}-r, t_{0}+\sigma\right] \rightarrow \mathbb{R}^{n}$ é uma função regrada, existe uma sequência em $\left[t_{0}, t\right], t_{0}<t_{1}<\ldots<t_{m}=t$, tal que $|y(\rho)-y(s)|<\epsilon$, quando $t_{k-1}<\rho, s<t_{k}, k=1, \ldots, m$ (veja Teorema 1.1).

Seja $\delta$ um calibre em $\left[t, t_{0}\right]$ tal que

$$
\delta(\tau)<\min \left\{\frac{t_{k}-t_{k-1}}{2}, k=1, \ldots, m\right\}, \quad \tau \in\left[t, t_{0}\right]
$$

$\mathrm{e}$

$$
\delta(\tau)<\min \left\{\left|\tau-t_{k}\right|,\left|\tau-t_{k-1}\right|, \tau \in\left[t_{k-1}, t_{k}\right], k=1, \ldots, m\right\}
$$

Esta escolha de $\delta$ garante que, se $\left(\tau_{k}, s_{k}\right)$ for uma divisão $\delta$-fina de $\left[t_{0}, t\right]$, então cada subintervalo $\left[s_{k-1}, s_{k}\right]$ irá conter no máximo um dos pontos $t_{0}, \ldots, t_{m}$ e, neste caso, $t_{k}$ será a marca correspondente deste intervalo. Pela continuidade da integral indefinida de Perron (veja Observação 1.10), podemos assumir, também, que

$$
\int_{t_{k}}^{t_{k}+\delta\left(t_{k}\right)} M(s)\left\|y_{s}-x\left(t_{k}\right)_{s}\right\| d s<\frac{\epsilon}{2 m+1}, \quad k=1, \ldots, m .
$$

Seja $D=\left(\tau_{k}, s_{k}\right)$ uma divisão $\delta$-fina de $\left[t_{0}, t\right]$ tal que

$$
\left\|\int_{t_{0}}^{t} D F(x(\tau), s)-\sum_{k=1}^{|D|}\left[F\left(x\left(\tau_{k}\right), s_{k}\right)-F\left(x\left(\tau_{k-1}\right), s_{k-1}\right)\right]\right\|<\epsilon .
$$


Por (4.2), temos

$$
\left[F\left(x\left(\tau_{k}\right), s_{k}\right)-F\left(x\left(\tau_{k-1}\right), s_{k-1}\right)\right](\vartheta)= \begin{cases}0, & \vartheta \in\left[t_{0}-r, s_{k-1}\right], \\ \int_{s_{k-1}}^{\vartheta} f\left(x\left(\tau_{k}\right)_{s}, s\right) d s, & \vartheta \in\left[s_{k-1}, s_{k}\right], \\ \int_{s_{k-1}}^{s_{k}} f\left(x\left(\tau_{k}\right)_{s}, s\right) d s, & \vartheta \in\left[s_{k}, t_{0}+\sigma\right] .\end{cases}
$$

para todo $k=1, \ldots,|D|$.

Seja $\vartheta \in\left[t_{0}, t\right]$. Então $\vartheta \in\left[s_{j-1}, s_{j}\right]$ para algum $j=1, \ldots,|D| \mathrm{e}$

$$
\begin{aligned}
\left|\sum_{k=1}^{|D|}\left[F\left(x\left(\tau_{k}\right), s_{k}\right)-F\left(x\left(\tau_{k-1}\right), s_{k-1}\right)\right](\vartheta)-\int_{t_{0}}^{\vartheta} f\left(y_{u}, u\right) d u\right| \\
=\mid \sum_{k=1}^{j-1}\left[F\left(x\left(\tau_{k}\right), s_{k}\right)-F\left(x\left(\tau_{k-1}\right), s_{k-1}\right)\right](\vartheta)-\int_{s_{k-1}}^{s_{k}} f\left(y_{u}, u\right) d u \\
\quad+\left[F\left(x\left(\tau_{j}\right), s_{j}\right)-F\left(x\left(\tau_{j-1}\right), s_{j-1}\right)\right](\vartheta)-\int_{s_{j-1}}^{\vartheta} f\left(y_{u}, u\right) d u \\
\quad+\sum_{k=j}^{|D|}\left[F\left(x\left(\tau_{k}\right), s_{k}\right)-F\left(x\left(\tau_{k-1}\right), s_{k-1}\right)\right](\vartheta) \mid \\
=\left|\sum_{k=1}^{j-1} \int_{s_{k-1}}^{s_{k}}\left[f\left(x\left(\tau_{k}\right)_{u}, u\right)-f\left(y_{u}, u\right)\right] d u+\int_{s_{j-1}}^{\vartheta}\left[f\left(x\left(\tau_{j}\right)_{u}, u\right)-f\left(y_{u}, u\right)\right] d u\right|
\end{aligned}
$$

Note que, se $u \leq \tau_{k}$, então $x\left(\tau_{k}\right)_{u}=y_{u} \mathrm{e}$, consequentemente,

$$
\int_{s_{k-1}}^{s_{k}}\left[f\left(x\left(\tau_{k}\right)_{u}, u\right)-f\left(y_{u}, u\right)\right] d u=\int_{\tau_{k}}^{s_{k}}\left[f\left(x\left(\tau_{k}\right)_{u}, u\right)-f\left(y_{u}, u\right)\right] d u
$$

para todo $k=1, \ldots, j-1 \mathrm{e}$

$$
\int_{s_{j-1}}^{\vartheta}\left[f\left(x\left(\tau_{j}\right)_{u}, u\right)-f\left(y_{u}, u\right)\right] d u= \begin{cases}0, & \vartheta \in\left[s_{j-1}, \tau_{j}\right] \\ \int_{\tau_{j}}^{s_{j}}\left[f\left(x\left(\tau_{j}\right)_{u}, u\right)-f\left(y_{u}, u\right)\right] d u, & \vartheta \in\left[\tau_{j}, s_{j}\right]\end{cases}
$$

Pela condição (B), obtemos

$$
\left|\int_{\tau_{k}}^{s_{k}}\left[f\left(x\left(\tau_{k}\right)_{u}, u\right)-f\left(y_{u}, u\right)\right] d u\right| \leq \int_{\tau_{k}}^{s_{k}} M(u)\left\|x\left(\tau_{k}\right)_{u}-y_{u}\right\| d u
$$


para todo $k=1, \ldots, j-1 \mathrm{e}$

$$
\left|\int_{\tau_{j}}^{\vartheta}\left[f\left(x\left(\tau_{j}\right)_{u}, u\right)-f\left(y_{u}, u\right)\right] d u\right| \leq \int_{\tau_{j}}^{\vartheta} M(u)\left\|x\left(\tau_{k}\right)_{u}-y_{u}\right\| d u .
$$

Se $u \in\left[\tau_{k}, s_{k}\right]$, então

$$
x\left(\tau_{k}\right)_{u}(\vartheta)=x\left(\tau_{k}\right)(u+\vartheta)= \begin{cases}y(u+\vartheta)=y_{u}(\vartheta), & u+\vartheta \leq \tau_{k}, \\ y\left(\tau_{k}\right), & \tau_{k} \leq u+\vartheta .\end{cases}
$$

para todo $k=1, \ldots, j-1$. Portanto, pela definição do calibre $\delta$, temos

$$
\left\|x\left(\tau_{k}\right)_{u}-y_{u}\right\|=\sup _{\rho \in\left[\tau_{k}, u_{k}\right]}\left|y(\rho)-y\left(\tau_{k}\right)\right| \leq \epsilon
$$

e, se a interseção de $\left[s_{k-1}, s_{k}\right]$ e $\left\{t_{0}, \ldots, t_{m}\right\}$ for vazia, então

$$
\int_{\tau_{k}}^{s_{k}} M(u)\left\|x\left(\tau_{k}\right)_{u}-y_{u}\right\| d u \leq \epsilon \int_{\tau_{k}}^{s_{k}} M(u) d u .
$$

para todo $k=1, \ldots, j-1$.

De maneira análoga, temos

$$
\int_{\tau_{j}}^{\vartheta} M(u)\left\|x\left(\tau_{j}\right)_{u}-y_{u}\right\| d u \leq \epsilon \int_{\tau_{j}}^{\vartheta} M(u) d u
$$

Se a interseção de $\left[s_{k-1}, s_{k}\right]$ e $\left\{t_{0}, \ldots, t_{m}\right\}$ não for vazia, então $\tau_{k}=t_{j}$, para algum $j \in\{1, \ldots, m\}$ e, neste caso,

$$
\int_{\tau_{k}}^{s_{k}} M(s)\left\|y_{s}-x\left(\tau_{k}\right)_{s}\right\| d s \leq \int_{t_{j}}^{t_{j}+\delta\left(t_{j}\right)} M(s)\left\|y_{s}-x\left(t_{j}\right)_{s}\right\| d s<\frac{\epsilon}{2 m+1} .
$$

Logo,

$$
\left|\sum_{k=1}^{|D|}\left[F\left(x\left(\tau_{k}\right), s_{k}\right)-F\left(x\left(\tau_{k-1}\right), s_{k-1}\right)\right](\vartheta)-\int_{t_{0}}^{\vartheta} f\left(y_{u}, u\right) d u\right| \leq \epsilon \int_{t_{0}}^{\vartheta} M(u) d u+\epsilon
$$


Por fim, como $\epsilon>0$ é arbitrário, obtemos

$$
\int_{t_{0}}^{t} D F(x(\tau), s)(\vartheta)=\int_{t_{0}}^{\vartheta} f\left(y_{u}, u\right) d u
$$

para todo $\vartheta \in\left[t_{0}, t\right]$.

Seguindo os mesmos passos acima, podemos provar o caso em que $\vartheta \in\left[t, t_{0}+\sigma\right]$.

Antes de apresentarmos o próximo lema, vamos recordar a definição do operador fundamental para EDO generalizada linear e, também, apresentar uma definição de operador solução para EDFs lineares.

Considere a EDO generalizada linear

$$
\frac{d x}{d \tau}=D[A(t) x]
$$

em que $A:[a, b] \rightarrow L(X)$. O operador fundamental associado a EDO generalizada linear (4.5) é o operador $U:[a, b] \times[a, b] \rightarrow L(X)$ que satisfaz a equação

$$
U(t, s)=I+\int_{s}^{t} d[A(r)] U(r, s)
$$

Além disso, se $\widetilde{x} \in X$, então $x:[a, b] \rightarrow X$ definido por $x(t)=U(t, s) \widetilde{x}$ será solução do problema de valor inicial

$$
\left\{\begin{array}{l}
\frac{d x}{d \tau}=D[A(t) x] \\
x(s)=\widetilde{x}
\end{array}\right.
$$

Considere, agora, a EDF linear

$$
\dot{y}=\mathscr{L}(t) y_{t}
$$

em que $\mathscr{L}(t) \in L\left(G\left([-r, 0], \mathbb{R}^{n}\right), \mathbb{R}^{n}\right)$ para todo $t \in\left[t_{0}, t_{0}+\sigma\right]$, e seja $\phi \in G\left([-r, 0], \mathbb{R}^{n}\right)$. Vejamos a definição de operador solução para EDFs lineares.

Definição 4.2. Seja $y:\left[t_{0}-r, t_{0}+\sigma\right] \rightarrow \mathbb{R}^{n}$ solução da EDF linear (4.6) com condição inicial 
$y_{s}=\phi$. Para $t, s \in\left[t_{0}, t_{0}+\sigma\right], t \geq s$, o o operador $T(t, s): G\left([-r, 0], \mathbb{R}^{n}\right) \rightarrow G\left([-r, 0], \mathbb{R}^{n}\right)$ definido por

$$
T(t, s) \phi=y_{t}, \quad t, s \in\left[t_{0}, t_{0}+\sigma\right], t \geq s
$$

será chamado operador solução da EDF linear (4.6).

Pela existência e unicidade de solução da EDF linear (3.1), o operador $T(t, s)$ safisfaz:

(i) $T(t, t)=I, t \in\left[t_{0}, t_{0}+\sigma\right]$ (propriedade de identidade),

(ii) $T(t, w) T(w, s)=T(t, s), t, w, s \in\left[t_{0}, t_{0}+\sigma\right]$ e $t \geq w \geq s$ (propriedade de semigrupo).

Esta definição de operador solução apresentada acima, restrita às funções contínuas, coincide com a definição de operador solução presente em [20] (veja Seção 6.2 em [20]).

Observação 4.3. Podemos generalizar a definição acima no seguinte sentido. Seja g: $\left[t_{0}-\right.$ $\left.r, t_{0}+\sigma\right] \rightarrow \mathbb{R}^{n}$ uma função dada e, para todo $s \in\left[t_{o}, t_{0}+\sigma\right]$ fixado, seja $y:\left[t_{0}-r, t_{0}+\sigma\right] \rightarrow \mathbb{R}^{n}$ solução da EDF linear

$$
\dot{y}=\mathscr{L}(t) y_{t}
$$

com condição inicial $y_{s}=g_{s}$. Então, para $t \in\left[t_{0}, t_{0}+\sigma\right], t \geq s$, definimos $T(t, s): G\left(\left[t_{o}-r, t_{0}+\right.\right.$ $\left.\sigma], \mathbb{R}^{n}\right) \rightarrow G\left([-r, 0], \mathbb{R}^{n}\right)$ por

$$
T(t, s) g=y_{t}
$$

Lema 4.4. Sejam y ex soluções dos problemas perturbados (4.1) e (4.3) respectivamente. Sejam $T(t, s)$ o operador solução da EDF linear (4.6) e U(t, s) o operador fundamental da EDO generalizada linear associada (4.5). Então, para $t_{0} \leq w \leq s \leq t \leq t_{0}+\sigma$, temos

$$
U(t, s)\left(\int_{t_{0}}^{w} D F(x(\tau), u)\right)(t)=T(t, s)\left(h(w)_{s}\right)(0)
$$


em que

$$
h(w)(\vartheta)= \begin{cases}0, & t_{0}-r \leq \vartheta \leq t_{0}, \\ \int_{t_{0}}^{\vartheta} f\left(y_{u}, u\right) d u, & t_{0} \leq \vartheta \leq w, \\ \int_{t_{0}}^{w} f\left(y_{u}, u\right) d u, & w \leq \vartheta \leq t_{0}+\sigma .\end{cases}
$$

Demonstração. Pelo Lema 4.1, temos

$$
\int_{t_{0}}^{w} D F(x(\tau), u)=h(w), \quad w \in\left[t_{0}, t_{0}+\sigma\right]
$$

Além disso, a Definição 4.2 implica que $T(t, s)\left(h(w)_{s}\right)(0)$ descreve a solução da EDF linear

$$
\left\{\begin{array}{l}
\dot{y}=\mathscr{L}(t) y_{t} \\
y_{s}=h(w)_{s}
\end{array}\right.
$$

e, pelo Teorema 2.15, $U(t, s) h(w)$ descreve a solução da EDO generalizada linear associada

$$
\left\{\begin{array}{l}
\frac{d x}{d \tau}=D[A(t) x] \\
x(s)=h(w),
\end{array}\right.
$$

em que $A$ é dada por (4.4). Então, Teorema 3.6 implica que

$$
U(t, s)\left(\int_{t_{0}}^{w} D F(x(\tau), u)\right)(t)=T(t, s)\left(h(w)_{s}\right)(0)
$$

para quaisquer $t, s, w \in\left[t_{0}, t_{0}+\sigma\right] \operatorname{com} w \leq s \leq t$.

O próximo resultado é novo e será útil para se obter uma fórmula da variação das constantes para a EDF linear perturbada (4.1).

Lema 4.5. Sejam y e x soluções dos problemas perturbados (4.1) e (4.3) respectivamente. Sejam $T(t, s)$ o operador solução da EDF linear (4.6) e U(t,s) o operador fundamental da EDO 
generalizada linear associada (4.5). Então, para $t_{0} \leq t \leq t_{0}+\sigma$, temos

$$
\int_{t_{0}}^{t} d_{s}[U(t, s)]\left(\int_{t_{0}}^{s} D F(x(\tau), u)\right)(t)=\int_{t_{0}}^{t} d_{s}[T(t, s)] h(s)(0)
$$

em que h é dada por (4.7) e as integrais envolvidas são no sentido da integral de KurzweilCauchy (veja Definição 1.17).

Demonstração. Dado $\epsilon>0$, a Definição 1.17 e o Lema 1.19 implicam que existe um calibre contínuo à esquerda $\delta$ em $\left[t_{0}, t\right]$ tal que, para toda divisão marcada à esquerda $\delta$-fina $D=$ $\left(s_{i-1},\left[s_{i-1}, s_{i}\right]\right)$ de $\left[t_{0}, t\right]$, temos

$$
\left\|\sum_{i=1}^{|D|}\left[U\left(t, s_{i}\right)-U\left(t, s_{i-1}\right)\right]\left(\int_{t_{0}}^{s_{i}-1} D F(x(\tau), u)\right)-\int_{t_{0}}^{t} d_{s}[U(t, s)]\left(\int_{t_{0}}^{s} D F(x(\tau), u)\right)\right\|<\epsilon .
$$

Pelo Lema 4.4, obtemos

$$
\sum_{i=1}^{|D|}\left[U\left(t, s_{i}\right)-U\left(t, s_{i-1}\right)\right]\left(\int_{t_{0}}^{s_{i}-1} D F(x(\tau), u)\right)(t)=\sum_{i=1}^{|D|}\left[T\left(t, s_{i}\right)-T\left(t, s_{i-1}\right)\right] h\left(s_{i-1}\right)(0) .
$$

e isto implica que

$$
\int_{t_{0}}^{t} d_{s}[U(t, s)]\left(\int_{t_{0}}^{s} D F(x(\tau), u)\right)(t)=\int_{t_{0}}^{t} d_{s}[T(t, s)] h(s)(0)
$$

o que completa a prova.

O próximo resultado nos dá um fórmula da variação das constantes para a EDF linear pertubada (4.1).

Teorema 4.6. Seja y solução do problema linear perturbado (4.1), onde supomos que as integrais envolvidas são no sentido da integral de Kurzweil-Cauchy. Seja T( $t, s)$ o operador solução da EDF linear (4.6). Então, para $t_{0} \leq t \leq t_{0}+\sigma$, temos

$$
y(t)=T\left(t, t_{0}\right) \phi(0)+\int_{t_{0}}^{t} f\left(y_{u}, u\right) d u-\int_{t_{0}}^{t} d_{s}[T(t, s)] h(s)(0) .
$$


em que hé definida por (4.7).

Demonstração. Dado $t \in\left[t_{0}, t_{0}+\sigma\right]$, os Teoremas 3.4 e 3.5 de [14] implicam que $y(t)=x(t)(t)$ e, pelos Teorema 2.22, obtemos

$$
x(t)(t)=U\left(t, t_{0}\right) \widetilde{x}(t)+\int_{t_{0}}^{t} D F(x(\tau), s)(t)-\int_{t_{0}}^{t} d_{s}[U(t, s)]\left(\int_{t_{0}}^{s} D F(x(\tau), u)\right)(t)
$$

em que $x$ é a solução do problema linear perturbado (4.3) e $U(t, s)$ é o operador fundamental da EDO generalizada linear (4.5).

Note que, pelos Teoremas 3.4 e 3.6,

$$
U\left(t, t_{0}\right) \widetilde{x}(t)=T\left(t, t_{0}\right) \phi(0)
$$

e, pelos Lemas 4.1 and 4.5,

$$
\int_{t_{0}}^{t} D F(x(\tau), s)(t)=\int_{t_{0}}^{t} f\left(y_{u}, u\right) d u
$$

$\mathrm{e}$

$$
\int_{t_{0}}^{t} d_{s}[U(t, s)]\left(\int_{t_{0}}^{s} D F(x(\tau), u)\right)(t)=\int_{t_{0}}^{t} d_{s}[T(t, s)] h(s)(0)
$$

Portanto,

$$
y(t)=T\left(t, t_{0}\right) \phi(0)+\int_{t_{0}}^{t} f\left(y_{u}, u\right) d u-\int_{t_{0}}^{t} d_{s}[T(t, s)] h(s)(0)
$$

e a prova está completa.

\subsection{Fórmula da variação das constantes para EDFs com im-}

\section{pulso}

Agora estamos interessados em obter uma fórmula da variação das constantes para EDFs lineares perturbadas que sofrem a ação de impulsos prefixados. Considere o problema de 
valor inicial

$$
\begin{cases}\dot{y}=\mathscr{L}(t) y_{t}+f\left(y_{t}, t\right), & t \neq t_{i} \\ \Delta y\left(t_{i}\right)=I_{k}\left(y\left(t_{k}\right)\right), & i=1,2, \ldots, m, \\ y_{t_{0}}=\phi, & \end{cases}
$$

em que as hipóteses sobre a equação (4.1) estão satisfeitas e, além disso, $t_{0}<t_{1}<\ldots<t_{k}<$ $\ldots<t_{m} \leq t_{0}+\sigma$ são momentos de impulsos conhecidos de antemão e

$$
\Delta y\left(t_{k}\right):=y\left(t_{k}+\right)-y\left(t_{k}-\right)=y\left(t_{k}+\right)-y\left(t_{k}\right), k=1,2, \ldots, m,
$$

isto é, a função $y$ é contínua à esquerda em $t=t_{k}$ e o limite lateral $y\left(t_{k}+\right)$ existe para $k=$ $1,2, \ldots, m$. Vamos supor, também, que os operadores de impulsos $I_{k}: \mathbb{R}^{n} \rightarrow \mathbb{R}^{n}, k=1, \ldots, m$, satisfaçam as seguintes condições:

(A') Existe uma constante $K_{1}>0$ tal que, para todo $k=1, \ldots, m$ e $x \in \mathbb{R}^{n}$, temos

$$
\left|I_{k}(x)\right| \leq K_{1}
$$

(B') Existe uma constante $K_{2}>0$ tal que, para todo $k=1, \ldots, m$ e $x, y \in \mathbb{R}^{n}$, vale

$$
\left|I_{k}(x)-I_{k}(y)\right| \leq K_{2}|x-y|
$$

Para $y \in G\left(\left[t_{0}-r, t_{0}+\sigma\right], \mathbb{R}^{n}\right)$ e $t \in\left[t_{0}, t_{0}+\sigma\right]$, seja

$$
H(y, t)=F(y, t)+J(y, t)
$$

em que $F$ é dada por (4.2) e, assim como em [14], $J: G\left(\left[t_{0}, t_{0}+\sigma\right], \mathbb{R}^{n}\right) \times\left[t_{0}-r, t_{0}+\sigma\right] \rightarrow G\left(\left[t_{0}-\right.\right.$ $\left.\left.r, t_{0}+\sigma\right], \mathbb{R}^{n}\right)$ é dada por

$$
J(y, t)(\vartheta)=\sum_{k=1}^{m} H_{t_{k}}(t) H_{t_{k}}(\vartheta) I_{k}\left(y\left(t_{k}\right)\right), \quad \vartheta \in\left[t_{0}-r, t_{0}+\sigma\right]
$$


com $H_{t_{k}}$ sendo a função de Heaviside contínua à esquerda concentrada em $t_{k}$.

Então o problema de valor inicial (4.8) se corresponde com a EDO generalizada perturbada

$$
\left\{\begin{array}{l}
\frac{d x}{d \tau}=D[A(t) x+H(x, t)]=D[A(t) x+F(x, t)+J(x, t)], \\
x\left(t_{0}\right)=\widetilde{x}
\end{array}\right.
$$

e pelo Teorema 2.22 e a correspondência de equações (veja [14], Teoremas 3.4 e 3.5), a solução $y$ de (4.8) pode ser descrita como

$$
\begin{aligned}
y(t)= & x(t)(t) \\
= & U\left(t, t_{0}\right) \widetilde{x}(t)+\int_{t_{0}}^{t} D H(x(\tau), s)(t)-\int_{t_{0}}^{t} d_{s}[U(t, s)]\left(\int_{t_{0}}^{s} D H(x(\tau), u)\right)(t) \\
= & U\left(t, t_{0}\right) \widetilde{x}(t)+\int_{t_{0}}^{t} D F(x(\tau), s)(t)-\int_{t_{0}}^{t} d_{s}[U(t, s)]\left(\int_{t_{0}}^{s} D F(x(\tau), u)\right)(t) \\
& +\int_{t_{0}}^{t} D J(x(\tau), s)(t)-\int_{t_{0}}^{t} d_{s}[U(t, s)]\left(\int_{t_{0}}^{s} D J(x(\tau), u)\right)(t)
\end{aligned}
$$

e, seguindo as ideias presentes nos Teoremas 3.4 e 3.5 de [14], obtemos

$$
\int_{t_{0}}^{t} D J(x(\tau), s)(\vartheta)=\sum_{k=1}^{m} H_{t_{k}}(t) H_{t_{k}}(\vartheta) I_{k}\left(y\left(t_{k}\right)\right)
$$

para quaisquer $t, \vartheta \in\left[t_{0}, t_{0}+\sigma\right]$, e pelos mesmos argumentos do Teorema 4.6, temos

$$
\begin{aligned}
y(t)= & T\left(t, t_{0}\right) \phi(0)+\int_{t_{0}}^{t} f\left(y_{u}, u\right) d u-\int_{t_{0}}^{t} d_{s}[T(t, s)] h(s)(0)+\sum_{k=1}^{m} I_{k}\left(y\left(t_{k}\right)\right) H_{t_{k}}(t) \\
& -\int_{t_{0}}^{t} d_{s}[T(t, s)]\left(\sum_{k=1}^{m} I_{k}\left(y\left(t_{k}\right)\right) H_{t_{k}}(s)\right)(0) .
\end{aligned}
$$

\subsection{Fórmula da variação das constantes para EDFNs em me-}

\section{dida}

Para finalizar as aplicações, veremos uma fórmula da variação das constantes para as equações diferenciais funcionais neutras em medida (EDFN em medida). Este tipo de equa- 
ção é mais geral que as equações vistas até o momento neste trabalho, porém as construções e ideias apresentadas até aqui podem ser traduzidas de maneira análoga. Iremos apenas apresentar a classe das EDFNs em medida e a fórmula da variação das constantes para a equação perturbada. Todos os resultados que vimos anteriormente para as EDFs lineares, assim como o os teoremas de correspondência, possuem análogos para EDFNs em medida. Veja, por exemplo, [8].

Considere a equação na forma

$$
D\left[N\left(y_{t}, t\right)\right]=f\left(y_{t}, t\right) D g
$$

em que $D\left[N\left(y_{t}, t\right)\right]$ e $D g(t)$ são as derivadas distribucionais de $N\left(y_{t}, t\right)$ e $g(t)$ respectivamente, no sentido de L. Schwartz. A equação (4.9) é dita equação diferencial funcional neutra em medida, ou simplesmente, EDFN em medida. Uma referência para EDFNs em medida é [8].

No que segue, vamos assumir que $f: G\left([-r, 0], \mathbb{R}^{n}\right) \times\left[t_{0}, t_{0}+\sigma\right] \rightarrow \mathbb{R}^{n}$ é dada por $f(y, t)=$ $\mathscr{L}(t) y$, em que $\mathscr{L}(t) \in L\left(G\left([-r, 0], \mathbb{R}^{n}\right), \mathbb{R}^{n}\right)$, para todo $t \in\left[t_{0}, t_{0}+\sigma\right]$. Além disso, vamos supor que a aplicação $t \mapsto \mathscr{L}(t) y_{t}$ é Kurzweil-Stieltjes integrável em $\left[t_{0}, t_{0}+\sigma\right]$ com respeito a função não decrescente $g:\left[t_{0}, t_{0}+\sigma\right] \rightarrow \mathbb{R}$.

Vamos supor que exista uma matriz $\mu: \mathbb{R} \times \mathbb{R} \rightarrow \mathbb{R}^{n \times n}$ tal que

$$
\mu(t, \theta)=0, \quad \theta \geq 0 \quad \text { e } \quad \mu(t, \theta)=\mu(t,-r), \quad \theta \leq-r .
$$

Suponha, também, que $\mu(t, \cdot)$ seja contínua à esquerda em $(-r, 0)$, de variação limitada em $[-r, 0]$ e que $\operatorname{var}_{[s, 0]} \mu(t, \cdot)$ tende a zero quando $s \rightarrow 0$, para todo $t \in \mathbb{R}$ fixado, e que o operador $N$ seja dado por

$$
N(\varphi, t)=\varphi(0)-\int_{-r}^{0} d_{\theta}[\mu(t, \theta)] \varphi(\theta)
$$

em que $\varphi \in G\left([-r, 0], \mathbb{R}^{n}\right)$. 
Portanto, usando (4.10) a equação

$$
D\left[N\left(y_{t}, t\right)\right]=\mathscr{L}(t) y_{t} D g
$$

a qual chamaremos de equação diferencial funcional neutra linear em medida (EDFN linear em medida), sujeita à condição inicial $y_{t_{0}}=\phi \in G\left([-r, 0], \mathbb{R}^{n}\right)$ pode ser reescrita na forma

$$
y(t)=\phi(0)+\int_{t_{0}}^{t} \mathscr{L}(s) y_{s} d g(s)+\int_{-r}^{0} d_{\theta}[\mu(t, \theta)] y(t+\theta)-\int_{-r}^{0} d_{\theta}\left[\mu\left(t_{0}, \theta\right)\right] \phi\left(t_{0}+\theta\right) .
$$

Em [8], podemos encontrar um resultado de correspondência entre soluções de EDFNs em medida e EDOs generalizadas. Usando os mesmos argumentos e hipóteses apresentados neste trabalho, pode-se provar que existe uma correspondência entre soluções de EDFNs lineares em medida e EDOs generalizadas lineares.

Se, assim como na Definição 4.2, $T(t, s)$ for o operador solução da EDFN linear em medida em (4.11), então, repetindo as construções e os resultados deste capítulo para o caso envolvendo equações neutras em medida, a solução da equação perturbada

$$
\left\{\begin{array}{l}
D\left[N\left(y_{t}, t\right)\right]=\left[\mathscr{L}(t) y_{t}+f\left(y_{t}, t\right)\right] D g \\
y_{t_{0}}=\phi
\end{array}\right.
$$

pode ser escrita na forma

$$
y(t)=T\left(t, t_{0}\right) \phi(0)+\int_{t_{0}}^{t} f\left(y_{u}, u\right) d g(u)-\int_{t_{0}}^{t} d_{s}[T(t, s)] l(s)(0)
$$

em que $l$ é dada por

$$
l(w)(\vartheta)= \begin{cases}0, & t_{0}-r \leq \vartheta \leq t_{0}, \\ \int_{t_{0}}^{\vartheta} f\left(y_{u}, u\right) d g(u), & t_{0} \leq \vartheta \leq w, \\ \int_{t_{0}}^{w} f\left(y_{u}, u\right) d g(u), & w \leq \vartheta \leq t_{0}+\sigma .\end{cases}
$$




\section{Referências Bibliográficas}

[1] S. M. Afonso, E. M. Bonotto, M. Federson, Š. Schwabik, Discontinuous local semiflows for Kurzweil equations leading to LaSalle's invariance principle for differential systems with impulses at variable times. J. Differential Equations 250 (2011), no. 7, 2969-3001.

[2] S. M. Afonso, E. M. Bonotto, M. Federson, L. P. Gimenes, Boundedness of solutions of retarded functional differential equations with variable impulses via generalized ordinary differential equations. Math. Nachr. 285 (2012), no. 5-6, 545-561.

[3] Z. Artstein, Topological dynamics of an ordinary differential equation and Kurzweil equations. J. Differential Equations 23 (1977), 224-243.

[4] M. Bohner, M. Federson, J. G. Mesquita, Continuous dependence for impulsive functional dynamic equations involving variable time scales. Appl. Math. Comput. 221 (2013), 383-393.

[5] B. Bongiorno, Un nuovo integrale per il problem delle primitive. Le Matematiche, 51, (1996), 299-313.

[6] R. Collegari, M. Federson and M. Frasson, Linear FDEs in the frame of generalized ODEs. Submetido.

[7] A. Denjoy, Sur les fonctions dérivées sommables. Bull. Soc. Math. Francése, 43, (1915), $161-248$. 
[8] M. Federson, M. Frasson, J. G. Mesquita, P. Tacuri, Measure neutral functional differential equations as generalized ODEs. Submetido.

[9] M. Federson, J. G. Mesquita, Non-periodic averaging principles for measure functional differential equations and functional dynamic equations on time scales involving impulses. J. Differential Equations 255 (2013), no. 10, 3098-3126.

[10] M. Federson, J. G. Mesquita, A. Slavík, Basic results for functional differential and dynamic equations involving impulses. Math. Nachr. 286 (2013), no. 2-3, 181-204.

[11] M. Federson, J. G. Mesquita, A. Slavík, Measure functional differential equations and functional dynamic equations on time scales. J. Differential Equations 252 (2012), no. 6, $3816-3847$.

[12] M. Federson, Š. Schwabik, A new approach to impulsive retarded differential equations: stability results. Funct. Differ. Equ. 16 (2009), no. 4, 583-607.

[13] M. Federson; Š. Schwabik, Stability for retarded functional differential equations. Ukraïn. Mat. Zh. 60 (2008), no. 1, 107-126; translation in Ukrainian Math. J. 60 (2008), no. $1,121-140$.

[14] M. Federson, S. Schwabik, Generalized ODE approach to impulsive retarded functional differential equations. Differential Integral Equations 19 (2006), no. 11, 1201-1234.

[15] M. Federson; P. Z. Táboas, Topological dynamics of retarded functional differential equations. J. Differential Equations 195 (2003), no. 2, 313-331.

[16] M. Federson, P. Z. Táboas, Impulsive retarded differential equations in Banach spaces via Bochner-Lebesgue and Henstock integrals. Nonlinear Anal. 50 (2002), no. 3, 389-407.

[17] D. Fraňková, Regulated functions. Math. Bohem. 116 (1991), no. 1, 20-59.

[18] R. A. Gordon, The integrals of Lebesgue, Denjoy, Perron and Henstock. American Mathematical Society, 1994. 
[19] R. Henstock, Lectures on the Theory of Integration. World Scientific, Singapore, 1988.

[20] J. K. Hale, S. M. V. Lunel, Introduction to Functional Differential Equations. SpringerVerlag, New York, 1993.

[21] C. S. Hönig, Volterra Stieltjes-integral equations. North-Holland Publ. Comp., Amsterdam, 1975.

[22] C. Imaz and Z. Vorel, Generalized ordinary differential equations in Banach spaces and applications to functional equations. Bol. Soc. Mat. Mexicana, 11 (1966), 47-59.

[23] J. Kurzweil, Generalized ordinary differential equations and continuous dependence on a parameter. Czechoslovak Math. J. 7(82) (1957), 418-448.

[24] J. Kurzweil, Generalized ordinary differential equations. Czechoslovak Math. J. 8(83) (1958), 360-388.

[25] J. Kurzweil, Unicity of solutions of generalized differential equations. Czechoslovak Math. J. 8(83) (1958), 502-509.

[26] J. Kurzweil, Addition to my paper "Generalized ordinary differential equations and continuous dependence on a parameter". Czechoslovak Math. J. 9(84) (1959), 564-573.

[27] J. Kurzweil, Problems which lead to a generalization of the concept of an ordinary nonlinear differential equation. 1963 Differential Equations and Their Applications, (Proc. Conf., Prague, 1962), pp. 65-76. Publ. House Czechoslovak Acad. Sci., Prague; Academic Press, New York.

[28] J. Kurzweil, Integration between the Lebesgue integral and Henstock-Kurzweil integral: its relation to local convex vector spaces. World Scientific, 2000.

[29] C. A. Martins, Existência de solução de equações integrais não lineares em escalas temporais sobre espaços de Banach. Dissertação de Mestrado, Universidade Estadual Paulista "Júlio de Mesquita Filho", 2013. 
[30] G. A. Monteiro, Generalized linear differential equations in a Banach space: continuous dependence on parameters and applications. Tese de Doutorado, Universidade de São Paulo, 2012.

[31] F. Oliva and Z. Vorel, Functional equations and generalized ordinary differential equations. Bol. Soc. Mat. Mexicana, 11 (1966), 40-46.

[32] O. Perron, Über den Integralbegriff. Heidelb. Ak. Sitzungsber. Nr. 14, S. 16 (1914).

[33] ̌. Schwabik, Generalized Ordinary Differential Equations. World Scientific, 1992.

[34] Š. Schwabik, Abstract Perron-Stieltjes integrals. Math. Bohem. 121 (1996), no. 4, 425447.

[35] Š. Schwabik, Linear Stieltjes integral equations in Banach spaces. Math. Bohem. 124 (1999), no. 4, 433-457.

[36] Š. Schwabik, Linear Stieltjes integral equations in Banach spaces II; Operator valued solutions. Math. Bohem. 125 (2000), no. 4, 431-454.

[37] M. Tvrdý, Differential and integral equations in the space of regulated functions, Mem. Differential Equations Math. Phys. 25 (2002), 1-104.

[38] M. Tvrdy, Linear integral equations in the space of regulated functions. Math. Bohem. 123 (1998), no. 2, 177-212. 